\title{
THE COLLATZ-WIELANDT QUOTIENT FOR PAIRS OF NONNEGATIVE OPERATORS
}

\author{
SHMUEL FRIEDLAND, CHICAGO
}

\begin{abstract}
In this paper we consider two versions of the Collatz-Wielandt quotient for a pair of nonnegative operators $A, B$ that map a given pointed generating cone in the first space into a given pointed generating cone in the second space. If the two spaces and two cones are identical, and $B$ is the identity operator then one version of this quotient is the spectral radius of $A$. In some applications, as commodity pricing, power control in wireless networks and quantum information theory, one needs to deal with the CollatzWielandt quotient for two nonnegative operators. In this paper we treat the two important cases: a pair of rectangular nonnegative matrices and a pair completely positive operators. We give a characterization of minimal optimal solutions and polynomially computable bounds on the Collatz-Wielandt quotient.
\end{abstract}

Keywords Perron-Frobenius theory, Collatz-Wielandt quotient, completely positive operators, commodity pricing, wireless networks, quantum information theory.

2010 Mathematics Subject Classification. 15A22, 15A45, 15B48, 15B57, 94A40

\section{INTRODUCTION}

The celebrated Perron-Frobenius theorem describes important spectral properties of a square matrix $A$ with nonnegative entries [31, 14, 15, 16. In particular, the spectral radius $\rho(A)$, (the maximum of absolute values of all eigenvalues of $A$ ), is an eigenvalue of $A$. Furthermore, to $\rho(A)$ correspond a nonnegative eigenvector $\mathbf{y}$ :

$$
A \mathbf{y}=\rho(A) \mathbf{y}, \quad \mathbf{y} \gtrless \mathbf{0},
$$

which is called the Perron-Frobenius eigenvector, abbreviated as PF-eigenvector. If $A$ is irreducible then $\mathbf{y}>\mathbf{0}$ and is unique up to multiplication by a positive scalar. There are many classical and recent books giving a full account of the Perron-Frobenius theory of nonnegative matrices for example [3, 12, 17, 23, 29, 30, 35. It is well known that PF-theory found innumerous applications in all sciences. See for example [1, 2, 33, 38] and references therein.

We denote by $\mathbb{R}^{m \times n} \supset \mathbb{R}_{+}^{m \times n}$ the sets of real valued and nonnegative valued $m \times n$ matrices respectively. $\left(\mathbb{R}^{m}=\mathbb{R}^{m \times 1} \supset \mathbb{R}_{+}^{m}=\mathbb{R}_{+}^{m \times 1}\right.$ the set of column vectors and the subset of nonnegative column vectors with $m$ coordinates respectively.) Let $[n]=\{1, \ldots, n\}, \frac{0}{0}=0, \frac{ \pm}{0}=\infty$.

One of the most applicable feature of PF-theory is the Collatz-Wielandt characterization of $\rho(A)$ for $A \in \mathbb{R}_{+}^{m \times m}[8,39$ :

$$
\inf _{\mathbf{x}=\left(x_{1}, \ldots, x_{m}\right)^{\top}>\mathbf{0}} \max _{i \in[m]} \frac{(A \mathbf{x})_{i}}{x_{i}}=\rho(A) .
$$

Theorem 6.4.5 in [12] gives a necessary and sufficient condiitons on $A$ that the above infimum is achieved for some positive $\mathbf{x}$. In a simple noiseless model in wireless netrworks $\frac{1}{\rho(A)}$ is the reception threshold [38, 33] and [12, §6.9].

Given a pair of nonsquare matrices $A, B \in \mathbb{R}^{m \times n}$ one can consider the generalized eigenvalue problem

$$
A \mathbf{x}=\lambda B \mathbf{x}, \quad A, B \in \mathbb{R}^{m \times n}, \lambda \in \mathbb{C} .
$$

In order to assure that one has a finite number of eigenvalues, one needs to assume that $\max (\operatorname{rank} A, \operatorname{rank} B)=n$, which implies that $m \geq n$. There is an extensive literature on this problem, see for example [9, 4, 7] and references there in. A first attempt to generalize Perron-Frobenius theory to (3), to the best knowledge of the author, is by Mangasarian [27. He showed the assumption that $B^{\top} \mathbf{y} \geq \mathbf{0}$ implies $A^{\top} \mathbf{y} \geq \mathbf{0}$ yields that (3) has a discrete and finite spectrum, and the eigenvalue with the largest absolute value is real, nonnegative and a corresponding eigenvector is nonnegative. 
The Perron-Frobenius theory was generalized to nonnegative operators $A$ with respect to a closed pointed generating cone $\mathbf{K}$ in finite and infinite dimensional Banach spaces [25, 24, 34, 3]. There is also a natural generalization of the Collatz-Wielandt characterizations to the spectral radius of $\rho(A)$ [10, 11].

The aim of this paper is to consider the Collatz-Wielandt type infmax problem for a pair of nonnegative operators $A, B: \mathbb{R}^{N_{1}} \rightarrow \mathbb{R}^{N_{2}}$, with respect to closed pointed generating cones $\mathbf{K}_{i} \subset \mathbb{R}^{N_{i}}$ for $i=1,2$ : $A \mathbf{K}_{1}, B \mathbf{K}_{1} \subseteq \mathbf{K}_{2}$. Denote by $\mathbf{K}_{i}^{o}$ the interior of $\mathbf{K}_{i}$. Let

$$
r(A, B, \mathbf{x})=\inf \left\{t, t \in[0, \infty], t B \mathbf{x}-A \mathbf{x} \in \mathbf{K}_{2}\right\} \quad \text { for } \mathbf{x} \in \mathbf{K}_{1} \backslash\{\mathbf{0}\} .
$$

We set $r(A, B, \mathbf{x})=\infty$ if $t B \mathbf{x}-A \mathbf{x} \notin \mathbf{K}_{2}$ for for each $t \geq 0$. Define

$$
\rho(A, B)=\inf \left\{r(A, B, \mathbf{x}), \mathbf{x} \in \mathbf{K}_{1}^{o}\right\} .
$$

In general, $\rho(A, B)$ can have any value in $[0, \infty]$. We call $\rho(A, B)$ the Collatz-Wielandt quotient.

We also consider the following variation of $\rho(A, B)$ :

$$
\hat{\rho}(A, B)=\inf \left\{r(A, B, \mathbf{x}), \mathbf{x} \in \mathbf{K}_{1} \backslash\{\mathbf{0}\}\right\} .
$$

We call $\hat{\rho}(A, B)$ the weak Collatz-Wielandt quotient. Clearly, $\hat{\rho}(A, B) \leq \rho(A, B)$. Even in the classical case, where $A \in \mathbb{R}_{+}^{m \times m}$ and $B$ is the identity matrix one may have the strict inequality $\hat{\rho}(A, I)<\rho(A, I)=\rho(A)$. A simple example is the following one. Assume that $A$ is a direct sum of $k$ irreducible matrices $A_{1}, \ldots, A_{k}$, where $\rho\left(A_{1}\right)>\rho\left(A_{2}\right)>\cdots>\rho\left(A_{k}\right)$. (So $A$ is block diagonal $\operatorname{diag}\left(A_{1}, \ldots, A_{k}\right)$.) Then $\rho(A, I)=\rho\left(A_{1}\right)$ and $\hat{\rho}(A, I)=\rho\left(A_{k}\right)$. See $\$ 3$. We show that if either $A$ or $B$ are positive then the Collatz-Wielandt quotient and the weak Collatz-Wielandt quotient are equal. Furthermore we have the following stability results. Suppose that we have two sequence of positive matrices $A_{l}$ and $B_{l}$ that converge to $A$ and $B$ respectively. Then $\lim _{l \rightarrow \infty} \rho\left(A, B_{l}\right)=\hat{\rho}(A, B)$, and $\lim _{l \rightarrow \infty} \rho\left(A_{l}, B\right)=\rho(A, B)$ provided that $B$ does not have a zero row. Thus the Collatz-Wielandt quotient and the weak Collatz-Wielandt quotient seem to be equally important quantities.

In the first part of this paper we consider the Collatz-Wielandt quotient for a pair of rectangular nonnegative matrices $A, B \in \mathbb{R}_{+}^{m \times n}$, i.e. :

$$
\rho(A, B):=\inf _{\mathbf{x}=\left(x_{1}, \ldots, x_{n}\right)^{\top}>\mathbf{0}} \max _{i \in[m]} \frac{(A \mathbf{x})_{i}}{(B \mathbf{x})_{i}} .
$$

(So $\mathbf{K}_{i}=\mathbb{R}_{+}^{N_{i}}$ for $i=1,2$ and $N_{1}=n, N_{2}=m$.)

We now give a simple model of commodity pricing, where the above Collatz-Wielandt ratio arises. (Another example in wireless networks discussed in [1, 2] is discussed in ,6]) Assume that we have $m$ producers of commodities which produce $n$ commodities. Each producer $i$ produces a subset of commodities $C(i) \subset[n]$. (We do not exclude the possibility that two producers produce the same commodity $j$.) Assume that the price of commodity $j$ is $x_{j}>0$. Then $\mathbf{x}=\left(x_{1}, \ldots, x_{n}\right)^{\top}>\mathbf{0}$ is the pricing vector. The expected value of the cost of the the producer $i$ for one unit of his products is $\sum_{j=1}^{n} a_{i j} x_{j}$. The expected value of the profit of the producer $i$ for one unit is $\sum_{j=1}^{n} b_{i j} x_{j}$. One can impose the obvious conditions that $a_{i j}=0$ if $j \in C(i)$, (the producer $i$ does not buy the commodity it produces), and $b_{i j}=0$ if $j \notin C(i)$, (the producer sell only the items it produces), Then the ratio of the profit to the expense for the producer $i$ is $\frac{(B \mathbf{x})_{i}}{(A \mathbf{x})_{i}}$. We call this ratio profit factor. In order that each producer will stay in business for the pricing vector $\mathbf{x}$ one needs to satisfy the minimum profit factor requirement: $\min _{i \in[m]} \frac{(B \mathbf{x})_{i}}{(A \mathbf{x})_{i}} \geq \beta$. Then the optimal pricing choice is the solution to the supremum problem

$$
\sup _{\mathbf{x}>\mathbf{0}} \min _{i \in[m]} \frac{(B \mathbf{x})_{i}}{(A \mathbf{x})_{i}}=\frac{1}{\rho(A, B)} .
$$

We are interested in a nontrivial case, where $\rho(A, B)<\infty$. It is easy to show that this inequality holds if and only the following condition is satisfied: For each zero row $i$ of $B$ the row $i$ of $A$ is zero.

We now summarize our results for the extremal problem (7). Assume that $\rho(A, B) \in(0, \infty)$. (It is easy to characterize the case $\rho(A, B)=0$.) Then there exists $\mathbf{y} \in \mathbb{R}_{+}^{n} \backslash\{\mathbf{0}\}$ such that $\rho(A, B)=r(A, B, \mathbf{y})$ with the following property: There exists a sequence $\mathbf{y}_{k}>\mathbf{0}$ for $k \in \mathbb{N}$ such that $\lim _{k \rightarrow \infty} \mathbf{y}_{k}=\mathbf{y}$ and $\lim _{k \rightarrow \infty} r\left(A, B, \mathbf{y}_{k}\right)=\rho(A, B)=r(A, B, \mathbf{y})$. Such $\mathbf{y}$ is called an optimal $\mathbf{y}$. An optimal vector $\mathbf{y}$ is called minimal optimal if $\mathbf{y}$ is an optimal vector, and there is no optimal vector $\mathbf{z}$ whose support is strictly contained in the support of $\mathbf{y}$. We call $\mathbf{y}$ a generalized Perron-Frobenius vector, abbreviated as GPF-eigenvector, if

$$
A \mathbf{y}=\rho(A, B) B \mathbf{y}, \quad \mathbf{y} \gtrless \mathbf{0} .
$$


Note that if (3) has an eigenvector $\mathbf{x}>\mathbf{0}$ with a corresponding $\lambda>0$, then $\rho(A, B) \leq \lambda$ and it is easy to give examples where $\rho(A, B)<\lambda$ and each optimal $\mathbf{y}$ is not a GPF-eigenvector. (See the example in the end of \$5) We next show, as briefly pointed in [2, that for any $\varepsilon \in(0,1)$, we can find one of the following: Either $\rho(A, B)<\varepsilon$ or we can find an approximation of $\rho(A, B, \varepsilon)$, such that $|\rho(A, B, \varepsilon)-\rho(A, B)| \leq \varepsilon \rho(A, B)$, in polynomial time. This follows from the well known fact that a solvability of linear system of equations is polynomial in the data 19,26 .

We show that each minimal optimal $\mathbf{y}$ has at most $m$ positive coordinates. The existence of an optimal $\mathbf{y}$ with at most $m+1$ positive coordinates in a general setting is shown in [2]. Furthermore, if there exists an optimal vector with $\ell \geq m$ positive coordinates, then the rank of the matrix $A^{\prime}-\rho(A, B) B^{\prime}$ is less than $m$. (Here $A^{\prime}, B^{\prime} \in \mathbb{R}_{+}^{m \times \ell}$ are the submatrices induced by $\ell$ positive entries of $\mathbf{y}$.) This result implies that for each minimal optimal $\mathbf{y}^{\prime}$ with $\ell$ positive coordinates, there is exists a minimal optimal $\mathbf{y}$ with the same support as $\mathbf{y}$ such that $(A \mathbf{y}-\rho(A, B) B \mathbf{y})_{i}=0$ for at least $\ell$ indices $i \in[m]$. That is, there exists a minimal optimal solution that is a GPF-eigenvector of the system $\tilde{A} \mathbf{y}=\rho(A, B) \tilde{B} \mathbf{y}$, where $\tilde{A}, \tilde{B}$ are the submatrices of $A, B$ obtained from $A, B$ by erasing a set of the of rows $\mathcal{I}$ in $A, B$ respectively. For the optimal commodity pricing model that we introduced above the above results have the following meaning: First each zero coordinate $j$ of $\mathbf{y}$ implies that the commodity $j$ is not produced. The producers corresponding to the set $\mathcal{I}$ have their profit ration above $\frac{1}{\rho(A, B)}$. For all other producers the profit ratio is $\frac{1}{\rho(A, B)}$. Similar results are shown for $\hat{\rho}(A, B)$.

We also give the following generalization of the main result in 2. Namely, if $B$ has no zero row and each column has one positive element, then there is an optimal solution which is a GPF-eigenvector. That is, in the wireless model of transmitters-receivers, where each receiver $i$ can obtain a signal from several transmitters, which can only transmit to the receiver $i$, there is a choice to pick exactly one transmitter $j(i)$. (However, if the system is not irreducible, as defined in 2], this choice would imply that some other transmitters to receiver $i^{\prime}$ should be shut off.)

The second part of this paper is a generalization of the above results to pairs of completely positive operators, which are frequently appear in quantum information theory as quantum channels. Denote by $\mathrm{H}_{n} \supset \mathrm{H}_{+, n} \supset \mathrm{H}_{+, 1, n}$ the real space of $n \times n$ Hermitian matrices, the cone of positive semidefinite matrices and the convex set of positive semidefinite matrices of trace one. Note that $\mathrm{H}_{+, n}$ is a pointed generating cone in $\mathrm{H}_{n} \equiv \mathbb{R}^{n^{2}}$. In quantum information theory (QIT), $\mathrm{H}_{+, 1, n}$ is the set of density matrices, (mixed states). Recall that $\mathcal{C}: \mathrm{H}_{n} \rightarrow \mathrm{H}_{m}$ is called a completely positive operator, abbreviated as CP-operator, if

$$
\mathcal{C}(X)=\sum_{j=1}^{k} T_{j} X T_{j}^{*}, \quad T_{j} \in \mathbb{C}^{m \times n}, j \in[k] .
$$

(Here $\mathbb{C}^{m \times n}$ is the space of $m \times n$ complex valued matrices and $T^{*}=\bar{T}^{\top}$ for $T \in \mathbb{C}^{m \times n}$.) Then $\mathcal{C}\left(\mathrm{H}_{+, n}\right) \subseteq$ $\mathrm{H}_{+, m}$, that is, $\mathcal{C}$ is a nonnegative operator with respect to the pair of cones $\mathrm{H}_{+, n}, \mathrm{H}_{+, m}$. In QIT $\mathcal{C}$ is called quantum channel if

$$
\sum_{j=1}^{k} T_{j}^{*} T_{j}=I_{n}
$$

That is, $\mathcal{C}$ is a quantum channel if and only if $\mathcal{C}$ is a $\mathrm{CP}$ trace preserving operator. In particular, $\mathcal{C}$ maps a density matrix to a density matrix. Quantum channel is one of the most significant notions in QIT 37, 21, 36, 20, 28, 22. The second main problem we discuss are $\rho(\mathcal{A}, \mathcal{B})$ and $\hat{\rho}(\mathcal{A}, \mathcal{B})$ for two CP-operators $\mathcal{A}, \mathcal{B}: \mathrm{H}_{+, n} \rightarrow \mathrm{H}_{+, m}$. The quantities $\rho(\mathcal{A}, \mathcal{B})$ and $\hat{\rho}(\mathcal{A}, \mathcal{B})$ could be viewed the quantum analog of the optimal commodity pricing assignment discussed above. We show that most of our results on $\hat{\rho}(A, B)$ generalize to $\hat{\rho}(\mathcal{A}, \mathcal{B})$, and some results on $\rho(A, B)$ generalize to $\rho(\mathcal{A}, \mathcal{B})$. We show that there exists weak optimal $Y \in \mathrm{H}_{+, n} \backslash\{0\}$ such that $r(\mathcal{A}, \mathcal{B}, Y)=\hat{\rho}(\mathcal{A}, \mathcal{B})$. A weak optimal $Y$ is called minimal if there is no optimal $Z$ such that range $Z$ is strictly contained in range $Y$. We show that a minimal weak optimal $Y$ has rank at most $m$. Assume that $Y^{\prime}$ is a minimal weak optimal with rank $\ell$. Then there exists a minimal weak optimal $Y$ satisfying range $Y^{\prime}=\operatorname{range} Y$, such that $\operatorname{rank}(\hat{\rho}(\mathcal{A}, \mathcal{B}) \mathcal{B}-\mathcal{A})(Y) \leq m-\ell$. In particular, if $\ell=m$ then $Y$ is a weak GPF-eigenvector. Assume that $\mathcal{B}$ is $\delta$-positive for a given rational $\delta>0$. (This assumption can be verified in polynomial time.) Then $\rho(\mathcal{A}, \mathcal{B})=\hat{\rho}(\mathcal{A}, \mathcal{B})$. Furthermore $\rho(\mathcal{A}, \mathcal{B})$ has an $\varepsilon \in(0,1)$ approximation in polynomial time in $\langle\mathcal{A}\rangle+\langle\mathcal{B}\rangle+\langle\delta\rangle+\langle\varepsilon\rangle$. (We need the assumption that $\mathcal{B}$ is $\delta$-positive because verifying 
SHMUEL FRIEDLAND, CHICAGO

the existence of a nonzero positive semidefinite matrix satisfying $(\mathcal{A}-t \mathcal{B})(X) \leq 0$ is a feasibility problem in semidefinite programming, which may be not polynomiallay solvable.)

We now survey briefly the content of the paper. Section 2 discusses basic properties of $\rho(A, B)$ and $\hat{\rho}(A, B)$. Section 3 discusses that classical case of the pair $A, B \in \mathbb{R}_{+}^{m \times n}$ where $m=n$ and $B$ is the idenity matrix $I$. We show that $\rho(A, I)=\rho(A)$. If $A$ is not irreducible than one may have the strict inequality $\hat{\rho}(A, I)<\rho(A, I)$. We characterize completely $\hat{\rho}(A, I)$. In Section 4 we give a polynomial time approximation algorithm to $\rho(A, B)$ and $\hat{\rho}(A, B)$. In Section 5 we give various properties of minimal optimal and minimal weak optimal vectors for the pair $A, B \in \mathbb{R}_{+}^{m \times n}$. In Section 6 we discuss WN-pairs $A, B \in \mathbb{R}_{+}^{m \times n}$ arising in wireless network. That is, $B$ has no zero row and one positive element in each column. Such pairs were introduced and studied in 1, 2, We give generalizations of the results in 1, 2, since we do not restrict ourselves to $S$-irreducible systems. Sections 7 , 8 and 9 are devoted to study of the Collatz-Wielandt quotients for pairs of completely positive operators.

\section{Preliminary Results}

For a positive integer $n$ let $\mathbf{1}_{n}=(1, \ldots, 1)^{\top} \in \mathbb{R}^{n}$. Let $S \subseteq[m], T \subseteq[n]$. Denote by $\mathbf{1}_{S}=\left(x_{1}, \ldots, x_{m}\right)^{\top} \in$ $\mathbb{R}_{+}^{m}$ the characterstic vector of $S$, i.e., $x_{i}=1$ if $i \in S$ and $x_{i}=0$ otherwise. So $\mathbf{1}_{\emptyset}=\mathbf{0}, \mathbf{1}_{[m]}=\mathbf{1}_{m}$. Assume that $F \in \mathbb{R}^{m \times n}$. Denote by $F(S, T)$ the matrix obtained from $F$ be deleting the rows of $F$ in the set $S$ and

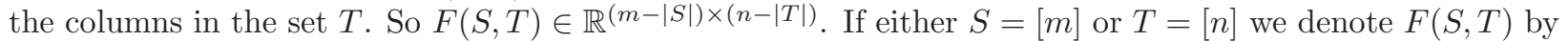
$\emptyset$. Denote by $F[S, T]$ the matrix $F([m] \backslash S,[n] \backslash T)$.

Assume that $A, B \in \mathbb{R}_{+}^{m \times n}$. For $\mathbf{x}=\left(x_{1}, \ldots, x_{n}\right)^{\top} \gtrless \mathbf{0}$ we define

$$
r(A, B, \mathbf{x})=\inf \{t, t \geq 0, A \mathbf{x} \leq t B \mathbf{x}\} .
$$

Note that $r(A, B, \mathbf{x}) \in[0, \infty]$. That is,$r(A, B, \mathbf{x})=\infty$ if and only if there exists $i \in[m]$ such that $(A \mathbf{x})_{i}>0$ and $(B \mathbf{x})_{i}=0$. Equivalently

$$
r(A, B, \mathbf{x})=\max \left\{\frac{(A \mathbf{x})_{i}}{(B \mathbf{x})_{i}}, i \in[m]\right\}
$$

Hence

$$
\rho(A, B)=\inf \{r(A, B, \mathbf{x}), \mathbf{x}>\mathbf{0}\}, \hat{\rho}(A, B)=\inf \left\{r(A, B, \mathbf{x}), \mathbf{x} \in \mathbb{R}_{+}^{n} \backslash\{\mathbf{0}\}\right\} .
$$

The following lemma is deduced straightforward.

Lemma 2.1. Assume that $A, B \in \mathbb{R}_{+}^{m \times n}$.

(1) $\rho(0, B)=\hat{\rho}(0, B)=0$.

(2) $\rho(A, B)=\infty$ if and only if $B$ has a zero row $i$, while the $i$-th row of $A$ is not zero.

(3) Let $S$ be a strict subset of $[m]$. Then for each $\mathbf{x} \geq \mathbf{0} r(A(S, \emptyset), B(S, \emptyset), \mathbf{x}) \leq r(A, B, \mathbf{x})$. In particular $\rho(A, B) \geq \rho(A(S, \emptyset), B(S, \emptyset))$ and $\hat{\rho}(A, B) \geq \hat{\rho}(A(S, \emptyset), B(S, \emptyset))$. Suppose furthermore that for each $i \in S$ the row $i$ of $A$ and $B$ are zero. Then for each $\mathbf{x} \geq \mathbf{0} r(A(S, \emptyset), B(S, \emptyset), \mathbf{x})=r(A, B, \mathbf{x})$. In particular, $\rho(A, B)=\rho(A(S, \emptyset), B(S, \emptyset))$ and $\hat{\rho}(A, B)=\hat{\rho}(A(S, \emptyset), B(S, \emptyset))$.

(4) Let $T$ be a strict subset of $[n]$ such that $A[[m], T]=B[[m], T]=0$. Then $\rho(A, B)=\rho(A(\emptyset, T), B(\emptyset, T))$ and $\hat{\rho}(A, B)=\hat{\rho}(A(\emptyset, T), B(\emptyset, T))$.

(5) Suppose that for each zero $i$ row of $B$ the row $i$ of $A$ is zero. Then $r\left(A, B, \mathbf{1}_{n}\right)<\infty$.

(6) There exists $\mathbf{x} \in \mathbb{R}_{+}^{n} \backslash\{\mathbf{0}\}$ such that $r(A, B, \mathbf{x})<\infty$ if and only if there exists a nonempty subset $T \subseteq[n]$ such that $r\left(A, B, \mathbf{1}_{T}\right)<\infty$.

(7) The weak Collatz-Wielandt quotient is positive if and only if the union of the supports of the rows of $A$ is $[n]$, i.e., $A^{\top} \mathbf{1}_{m}>\mathbf{0}$.

(8) Assume that $A_{1}, B_{1} \in \mathbb{R}_{+}^{m \times n}$ and $A_{1} \leq A, B \leq B_{1}$ then $\rho\left(A_{1}, B_{1}\right) \leq \rho(A, B)$ and $\hat{\rho}\left(A_{1}, B_{1}\right) \leq$ $\hat{\rho}(A, B)$.

The following lemma gives a lower bound on $\hat{\rho}(A, B)$ :

Lemma 2.2. Let $A=\left[a_{i j}\right], B=\left[b_{i j}\right] \in \mathbb{R}_{+}^{m \times n}$. Then

$$
\hat{\rho}(A, B) \geq \min _{j \in[n]} \frac{\sum_{i=1}^{m} a_{i j}}{\sum_{i=1}^{m} b_{i j}} .
$$


Proof. Clearly, it is enough to assume that $\hat{\rho}(A, B)<\infty$. Assume that $\mathbf{x} \gtrless \mathbf{0}$ and $r(A, B, \mathbf{x})<\infty$. Observe that

$$
r(A, B, \mathbf{x}) \geq \frac{\sum_{i=1}^{m}(A \mathbf{x})_{i}}{\sum_{i=1}^{m}(B \mathbf{x})_{i}}=\frac{\mathbf{1}_{m} A \mathbf{x}}{\mathbf{1}_{m} B \mathbf{x}}=\frac{\sum_{j=1}^{n}\left(\mathbf{1}_{m} A\right)_{j} x_{j}}{\sum_{j=1}^{n}\left(\mathbf{1}_{m} B\right)_{j} x_{j}} \geq \min _{j \in[n]} \frac{\left(\mathbf{1}_{m} A\right)_{j}}{\left(\mathbf{1}_{m} B\right)_{j}} .
$$

Lemma 2.3. Assume that $A, B \in \mathbb{R}_{+}^{m \times n}$. Let $\mathcal{I} \subseteq[m]$ be the set of the zero rows of $B$. Denote by $T$ be the union of the the supports of the the rows $i \in \mathcal{I}$ of $A$. Then one of the following conditions holds

(1) If $\mathcal{I}=\emptyset$ then $\rho(A, B) \leq r\left(A, B, \mathbf{1}_{n}\right)<\infty$.

(2) If $T=[n]$ then $\hat{\rho}(A, B)=\infty$

(3) Assume that $\mathcal{I} \neq \emptyset$ and $T$ is a strict subset of $[n]$, Then $\hat{\rho}(A, B)=\hat{\rho}(A(\mathcal{I}, T), B(\mathcal{I}, T))$.

Proof. (1) If $\mathcal{I}=\emptyset$ then $\left(B \mathbf{1}_{n}\right)_{i}>0$ for each $i$ and $r\left(A, B, \mathbf{1}_{n}\right)=\max _{i \in[m]} \frac{\left(A \mathbf{1}_{n}\right)_{i}}{\left(B \mathbf{1}_{n}\right)_{i}}<\infty$.

(2) Suppose that $T=[n]$. Let $\mathbf{x} \gtrless \mathbf{0}$. Then there exists $i \in \mathcal{I}$ such that $(A \mathbf{x})_{i}>0$ and $(B \mathbf{x})_{i}=0$. Hence $r(A, B, \mathbf{x})=\infty$ which yields that $\hat{\rho}(A, B)=\infty$.

(3) Suppose that $\mathcal{I} \neq \emptyset, T \subset[n]$. Let $\mathbf{x} \geqslant \mathbf{0}$ and assume that ( $\operatorname{supp} \mathbf{x}) \cap T \neq \emptyset$. Then there exists $i \in \mathcal{I}$ such that $(A \mathbf{x})_{i}>0$. As $(B \mathbf{x})_{i}=0$ it follows that $r(A, B, \mathbf{x})=\infty$. Hence to determine $\hat{\rho}(A, B)$ is enough to consider $\inf \{r(A, B, \mathbf{x}), \mathbf{x} \geqslant \mathbf{0}$, supp $\mathbf{x} \subseteq[n] \backslash T\}$. Note that if supp $\mathbf{x} \subseteq[n] \backslash T$ then $(A \mathbf{x})_{i}=(B \mathbf{x})_{i}=0$ for $i \in \mathcal{I}$. Therefore $\hat{\rho}(A, B)=\hat{\rho}(A(\mathcal{I}, T), B(\mathcal{I}, T))$.

The above lemma gives rise to a polynomial time algorithm to check if $\hat{\rho}(A, B)$ is finite or infinite:

Algorithm 2.4. Given $A=\left[a_{i j}\right], B=\left[b_{i j}\right] \in \mathbb{R}_{+}^{m \times n}$ set $S=[n], \mathcal{I}=\mathcal{J} \subseteq[m]$ the set of zero rows of $B$ and $T$ the union of the the supports of the the rows $i \in \mathcal{I}$ of $A$;

While $\mathcal{I} \neq \emptyset$ and $T \neq S$

Replace $A, B, S$ by $A(\mathcal{I}, T), B(\mathcal{I}, T), S \backslash T$;

Replace $\mathcal{I}$ by the set of zero rows of $B$;

Replace $T$ by the union of the the supports of the rows $i \in \mathcal{I}$ of $A$;

Replace $\mathcal{J}$ by $\mathcal{I} \cup \mathcal{J}$;

Else

If $\mathcal{I}=\emptyset$ then $\hat{\rho}(A, B) \leq r\left(A, B, 1_{S}\right)<\infty$ and stop;

If $T=S$ then $\hat{\rho}(A, B)=\infty$ and stop;

Denote by $\Pi_{n} \subset \mathbb{R}_{+}^{n}$ the set of probability vectors on $\mathbb{R}_{+}^{n}$. Let $\Pi_{n}^{o}$ be the interior of $\Pi_{n}$, i.e., all probability vectors with positive coordinates.

We now discuss some properties of $\rho(A, B)$ and $\hat{\rho}(A, B)$.

Lemma 2.5. Let $A, B \in \mathbb{R}_{+}^{m \times n}$.

(1) The function $r(A, B, \mathbf{x})$ is lower semicontinuous on $\mathbb{R}_{+}^{m \times n} \times \mathbb{R}_{+}^{m \times n} \times\left(\mathbb{R}_{+}^{n} \backslash\{\mathbf{0}\}\right)$.

(2) Let $T \subset[n]$ be a nonempty subset such that $B \mathbf{1}_{T}>\mathbf{0}$. Assume that $\mathbf{x} \in \mathbb{R}_{+}^{n}, \sup \mathbf{y}=T$. Suppose that the sequence $\mathbf{0}<\mathbf{x}_{k} \in \mathbb{R}^{n}, k \in \mathbb{N}$ converges to $\mathbf{x}$. Then $\lim _{k \rightarrow \infty} r\left(A, B, \mathbf{x}_{k}\right)=r(A, B, \mathbf{x})$. In particular $\rho(A[[m], T], B[[m], T]) \geq \rho(A, B)$.

(3) Assume that

$A=\left[\begin{array}{ll}A_{1} & A_{2}\end{array}\right], A_{1}=\left[\begin{array}{c}A_{11} \\ 0\end{array}\right], A_{2}=\left[\begin{array}{l}A_{12} \\ A_{22}\end{array}\right], B=\left[\begin{array}{ll}B_{1} & B_{2}\end{array}\right], B_{1}=\left[\begin{array}{c}B_{11} \\ 0\end{array}\right], B_{2}=\left[\begin{array}{c}B_{12} \\ B_{22}\end{array}\right]$,

where $A_{11}, B_{11} \in \mathbb{R}^{m^{\prime} \times \ell}, 1 \leq m^{\prime}<m, 1 \leq \ell<n$, and $B_{11} \mathbf{1}_{\ell}>\mathbf{0}$. Suppose that $\rho\left(A_{11}, B_{11}\right)<$ $\rho(A, B)$. Then $\rho\left(A_{22}, B_{22}\right)=\rho(A, B)$.

Proof. (1) Suppose that $A_{k}, B_{k} \in \mathbb{R}_{+}^{m \times n}, \mathbf{x}_{k} \in \mathbb{R}_{+}^{n} \backslash\{\mathbf{0}\}, k \in \mathbb{N}$ and assume that

$$
\lim _{k \rightarrow \infty} A_{k}=A, \quad \lim _{k \rightarrow \infty} B_{k}=B, \quad \lim _{k \rightarrow \infty} \mathbf{x}_{k}=\mathbf{x} \in \mathbb{R}_{+}^{n} \backslash\{\mathbf{0}\} .
$$

Suppose that $t=\liminf _{k \rightarrow \infty} r\left(A_{k}, B_{k}, \mathbf{x}_{k}\right) \in[0, \infty]$. If $t=\infty$ then $r(A, B, \mathbf{x}) \leq t$. Assume that $t \in[0, \infty)$. By passing to subsequences we can assume without loss of generality that $\lim _{k \rightarrow \infty} r\left(A_{k}, B_{k}, \mathbf{x}_{k}\right)=t$. As $A_{k} \mathbf{x}_{k} \leq r\left(A_{k}, B_{k}, \mathbf{x}_{k}\right) \mathbf{x}_{k}$ we deduce that $A \mathbf{x} \leq t B \mathbf{x}$. Hence $r(A, B, \mathbf{x}) \leq t$. 
(2) As $B \mathbf{1}_{T}>\mathbf{0}$ and $\sup \mathbf{x}=T$ we deduce that $B \mathbf{x}>\mathbf{0}$. Hence

$$
\rho(A, B) \leq \lim _{k \rightarrow \infty} r\left(A, B, \mathbf{x}_{k}\right)=\lim _{k \rightarrow \infty} \max _{i \in[m]} \frac{\left(A \mathbf{x}_{k}\right)_{i}}{\left(B \mathbf{x}_{k}\right)_{i}}=\max _{i \in[m]} \frac{(A \mathbf{x})_{i}}{(B \mathbf{x})_{i}}=r(A, B, \mathbf{x}) .
$$

Let $\mathbf{z} \in \mathbb{R}^{|T|}$ be the projection of $\mathbf{x}$ on its support. Then $\mathbf{z}>\mathbf{0}$ and $r(A, B, \mathbf{x})=r(A[[m], T], B[[m], T], \mathbf{z})$. Hence $\rho(A[[m], T], B[[m], T]) \geq \rho(A, B)$.

(3) Assume that $\rho\left(A_{11}, B_{11}\right)<t_{0}=\rho(A, B)$. Suppose to the contrary that $\rho\left(A_{22}, B_{22}\right)<t_{0}$. Let $\mathbf{0}<\mathbf{z} \in$ $\mathbb{R}^{\ell}, \mathbf{0}<\mathbf{u} \in \mathbb{R}^{n-\ell}$ such that $r\left(A_{11}, B_{11}, \mathbf{z}\right)<t_{0}, r\left(A_{22}, B_{22}, \mathbf{u}\right)<t_{0}$. For $s>0$ let $\mathbf{x}(s)=\left(\mathbf{z}^{\top}, s \mathbf{u}^{\top}\right)^{\top}$. As $B_{11} \mathbf{z}>\mathbf{0}$ there exists $K>0$ such that $B_{11} \mathbf{z} \geq K A_{12} \mathbf{u}$. Then

$$
\begin{aligned}
& A_{11} \mathbf{z}+s A_{12} \mathbf{u} \leq r\left(A_{11}, B_{11}, \mathbf{z}\right) B_{11} \mathbf{z}+s K B_{11} \mathbf{z}=\left(r\left(A_{11}, B_{11}+s K\right) B_{11} \mathbf{z}\right. \\
& A_{22} s \mathbf{u} \leq r\left(A_{22}, B_{22}\right) s \mathbf{u} .
\end{aligned}
$$

Thus for small enough $s$ we have the inequality $r(A, B, \mathbf{x}(s))<t_{0}$, which is a contradiction.

Lemma 2.6. Let $m, n \geq 1$ be integers. Assume that $A, B \in \mathbb{R}_{+}^{m \times n}$. Then

(1) There exists $\mathbf{y} \in \mathbb{R}_{+}^{n} \backslash\{\mathbf{0}\}$ such that $\hat{\rho}(A, B)=r(A, B, \mathbf{y})$. (Such $\mathbf{y}$ is called a weak optimal $\mathbf{y}$.)

(2) There exists $\mathbf{y} \in \mathbb{R}_{+}^{n} \backslash\{\mathbf{0}\}$ such that $\rho(A, B)=r(A, B, \mathbf{y})$ with the following property: There exist a sequence $\mathbf{y}_{k}>\mathbf{0}$ for $k \in \mathbb{N}$ such that $\lim _{k \rightarrow \infty} \mathbf{y}_{k}=\mathbf{y}$ and $\lim _{k \rightarrow \infty} r\left(A, B, \mathbf{y}_{k}\right)=\rho(A, B)=r(A, B, \mathbf{y})$. (Such $\mathbf{y}$ is called an optimal $\mathbf{y}$.)

Proof. (1) If $\hat{\rho}(A, B)=\infty$ then each $\mathbf{x} \in \Pi_{n}$ is weak optimal. Assume that $\hat{\rho}(A, B)<\infty$. Choose a sequence of $\mathbf{y}_{k} \in \Pi_{n}$, such that $t_{k}:=r\left(A, B, \mathbf{y}_{k}\right) \in(0, \infty), k \in \mathbb{N}, t_{k} \geq t_{k+1}$ for $k \in \mathbb{N}$, such that $\lim _{k \rightarrow \infty}=\hat{\rho}(A, B)$. Pick up a subsequence of $\mathbf{y}_{k}$ which converges to $\mathbf{y} \in \Pi_{n}$. Part (1) of Lemma 2.5 yields that $r(A, B, \mathbf{y}) \leq \hat{\rho}(A, B)$. Thus $r(A, B, \mathbf{y})=\hat{\rho}(A, B)$ and $\mathbf{y}$ is weak optimal.

(2) If $\rho(A, B)=\infty$ then each $\mathbf{x} \in \Pi_{n}^{o}$ is optimal. Assume that $\rho(A, B)<\infty$. Without loss of generality we may assume that $B$ does not have a zero row. We show by induction on $n$ that there exists an optimal y. For $n=1$ this claim is trivial. Assume that the claim holds for $n \leq N$. Suppose that $n=N+1$. There exists a sequence of $\mathbf{y}_{k} \in \Pi_{n}^{o}$, such that $t_{k}:=r\left(A, B, \mathbf{y}_{k}\right) \in(0, \infty), k \in \mathbb{N}, t_{k} \geq t_{k+1}$ for $k \in \mathbb{N}$, such that $\lim _{k \rightarrow \infty}=\rho(A, B)$. Pick up a subsequence of $\mathbf{y}_{k}$ which converges to $\mathbf{w} \in \Pi_{n}$. Part (1) of Lemma 2.5 yields that $r(A, B, \mathbf{w}) \leq \rho(A, B)$. If $\rho(A, B)=0$ we deduce that $\mathbf{w}$ is optimal. Assume that $\rho(A, B)>0$. If $r(A, B, \mathbf{w})=\rho(A, B)$ then $\mathbf{w}$ is minimal. Assume that $r(A, B, \mathbf{w})<\rho(A, B)$. Hence $T=\operatorname{supp} \mathbf{w}$ is a strict subset of $[n]$. Furthermore part (2) of Lemma 2.5 yields that $B \mathbf{1}_{T}$ is not positive.

By relabeling the elements of $[n]$ we can assume that $T=[\ell]$ for some $\ell \in[n-1]$. Let $\mathcal{K}=\{\ell+1, \ldots, n\}$ and denote

$$
A_{1}=A[[m],[\ell]], B_{1}=B[[m],[\ell]], A_{2}=A[[m], \mathcal{K}], B_{2}=B[[m], \mathcal{K}] .
$$

Let $\mathcal{I}$ be the set of zero rows of $B_{1}$. As $B_{1} \mathbf{1}_{\ell}$ is not positive $|\mathcal{I}| \geq 1$. As $r(A, B, \mathbf{w})<\infty$ we deduce that $\mathcal{I}$ is a subset of zero rows of $A_{1}$. Let $\mathbf{0}<\mathbf{z} \in \mathbb{R}^{\ell}$ be the projection of $\mathbf{w}$ on its support. Relabel the rows of $A$ and $B$ such that $\mathcal{I}=\left\{m^{\prime}+1, \ldots, m\right\}$.

Assume first that $m^{\prime}=0$, i.e., $\mathcal{I}=[m]$. So $A_{1}=B_{1}=0$. Part 4 of of Lemma 2.1 yields that $\rho(A, B)=\rho\left(A_{2}, B_{2}\right)$. As $|\mathcal{K}|=n-\ell<n$ we can apply the induction hypothesis to $\left(A_{2}, B_{2}\right)$ to deduce the existence of an optimal $\mathbf{u} \in \Pi_{n-\ell}, \operatorname{supp} \mathbf{y} \subseteq \mathcal{K}$. That is, there exists a sequence $\mathbf{0}<\mathbf{u}_{k} \in \mathbb{R}^{n-\ell}, k \in \mathbb{N}$ such that $\lim _{k \rightarrow \infty} \mathbf{u}_{k}=\mathbf{u}$ and $\lim _{k \rightarrow \infty} r\left(A_{2}, B_{2}, \mathbf{u}_{k}\right)=\rho\left(A_{2}, B_{2}\right)$. Choose a sequence $\mathbf{0}<\mathbf{z}_{k} \in \mathbb{R}^{\ell}$ such that $\lim _{k \rightarrow \infty} \mathbf{z}_{k}=\mathbf{0}$. Let $\mathbf{v}_{k}=\left(\mathbf{z}_{k}^{\top}, \mathbf{u}_{k}^{\top}\right)>\mathbf{0}$ for $k \in \mathbb{N}$. Clearly $r\left(A, B, \mathbf{v}_{k}\right)=r\left(A_{2}, B_{2}, \mathbf{u}_{k}\right)$. Hence $\left.\mathbf{y}=\left(\mathbf{0}^{\top}, \mathbf{u}^{\top}\right)^{\top}\right)$ is optimal.

Assume that $m^{\prime}=m-|\mathcal{I}| \in[m-1]$. It now follows that the conditions of part (3) of Lemma 2.5 holds. The induction hypothesis yields that there exists $\mathbf{u} \in \mathbb{R}_{+}^{n-\ell} \backslash\{\mathbf{0}\}$ such that $r\left(A_{22}, B_{22}, \mathbf{u}\right)=\rho\left(A_{22}, B_{22}\right)=$ $\rho(A, B)$. Furthermore, there exists a sequence $\mathbf{0}<\mathbf{v}_{k} \in \mathbb{R}^{n-\ell}, k \in \mathbb{N}$ such that $\lim _{k \rightarrow \infty} \mathbf{v}_{k}=\mathbf{v}$ and $\lim _{k \rightarrow \infty} r\left(A_{2}, B_{2}, \mathbf{v}_{k}\right)=\rho\left(A_{2}, B_{2}\right)$. For $s>0$ let $\mathbf{v}(s)=\left(\mathbf{z}^{\top}, s \mathbf{v}\right)^{\top}$. As in the proof of of part (3) of Lemma 2.5 we deduce $r(A, B, \mathbf{v}(s)) \leq \max \left(r\left(A_{11}, B_{11}, \mathbf{z}\right)+K s, \rho(A, B)\right)$. Choose $s_{0}>0$ such that $r\left(A_{11}, B_{11}, \mathbf{z}\right)+$ $K s_{0}<\rho(A, B)$. Then $r\left(A, B, \mathbf{v}\left(s_{0}\right)\right)=\rho\left(A_{2}, B_{2}\right)=\rho(A, B)$. Set $\mathbf{v}_{k}=\left(\mathbf{z}^{\top}, s_{0} \mathbf{u}_{k}^{\top}\right)^{\top}>\mathbf{0}, k \in \mathbb{N}$. So $\lim _{k \rightarrow \infty} \mathbf{v}_{k}=\mathbf{v}\left(t_{0}\right)$. Choose $K^{\prime}>K$ such that $r\left(A_{11}, B_{11}, \mathbf{z}\right)+K^{\prime} s_{0}<\rho(A, B)$. As $\lim _{k \rightarrow \infty} \mathbf{u}_{k} \rightarrow \mathbf{u}$ it follows that there exist $N$ such that $A_{12} \mathbf{u}_{k} \leq K^{\prime} B \mathbf{z}$ for $k>N$. Therefore $r\left(A, B, \mathbf{v}_{k}\right)=\rho\left(A_{2}, B_{2}, \mathbf{u}_{k}\right)$ for $k>N$. This implies that $\mathbf{v}\left(s_{0}\right)$ is optimal. 
We call $\mathbf{y}$ a minimal (weak) optimal if $\mathbf{y}$ is (weak) optimal and there is no (weak) optimal $\mathbf{w}$ such that the support of $\mathbf{w}$ is strictly contained in the support of $\mathbf{y}$. A vector $\mathbf{y} \geq \mathbf{0}$ is called a weak GPF-eigenvector if $A \mathbf{y}=\hat{\rho}(A, B) \mathbf{y}$.

The next lemma discusses connections between $\rho(A, B)$ and $\hat{\rho}(A, B)$.

Lemma 2.7. Let $A, B \in \mathbb{R}_{+}^{m, n}$. Then

(1) $\hat{\rho}(A, B) \leq \rho(A, B)$.

(2) Let $\mathbf{y}$ be weak optimal. If either $A \mathbf{y}>\mathbf{0}$ or $B \mathbf{y}>\mathbf{0}$ then $\rho(A, B)=\hat{\rho}(A, B)$ and $\mathbf{y}$ is optimal.

(3) Assume that either $A>0$ or $B>0$. Then $\rho(A, B)=\hat{\rho}(A, B)$ and each weak optimal $\mathbf{y}$ is optimal.

(4) Assume that $0<B_{l} \in \mathbb{R}^{m \times n}$ for $l \in \mathbb{N}$ and $\lim _{l \rightarrow \infty} B_{l}=B$. Then $\lim _{l \rightarrow \infty} \rho\left(A, B_{l}\right)=\hat{\rho}(A, B)$.

(5) Assume that $0<A_{l} \in \mathbb{R}^{m \times n}$ for $l \in \mathbb{N}$ and $\lim _{l \rightarrow \infty} A_{l}=A$. If $B$ does not have a zero row then $\lim _{l \rightarrow \infty} \rho\left(A_{l}, B\right)=\rho(A, B)$.

Proof. (1) The inequality $\hat{\rho}(A, B) \leq \rho(A, B)$ is clear.

(2) Let $\mathbf{y} \in \Pi_{n}$ be weak optimal. So $r(A, B, \mathbf{y})=\hat{\rho}(A, B)$. Assume first that $B \mathbf{y}>0$. For $t>0$ define $\mathbf{y}(t)=\mathbf{y}+t \mathbf{1}_{n}$. Note that $\lim _{t \searrow 0} \mathbf{y}(t)=\mathbf{y}$. As $(B \mathbf{y})_{i}>0$ for $i \in[n]$ it follows that $\lim _{t \searrow_{0}} \frac{(A \mathbf{y}(t))_{i}}{(B \mathbf{y}(t))_{i}}=\frac{(A \mathbf{y})_{i}}{(B \mathbf{y})_{i}}$. Hence

$$
\rho(A, B) \leq \lim _{t \searrow 0} r(A, B, \mathbf{y}(t))=r(A, B, \mathbf{y})=\hat{\rho}(A, B) \Rightarrow \rho(A, B)=\hat{\rho}(A, B),
$$

and $\mathbf{y}$ is optimal.

Assume second that $A \mathbf{y}>0$. Suppose that $B$ has a zero row. Then $\rho(A, B)=\hat{\rho}(A, B)=\infty$ and $\mathbf{y}$ is optimal. Assume now that $B$ does not have a zero row. Then $\rho(A, B)<\infty$. As $A \mathbf{y} \leq \hat{\rho}(A, B) B \mathbf{y}$ it follows that $\hat{\rho}(A, B)>0$ and $B \mathbf{y}>\mathbf{0}$. Therefore the first case yields $\rho(A, B)=\hat{\rho}(A, B)$ and $\mathbf{y}$ is optimal.

(3) This claim follows from part 2.

(4) Assume that we have a sequence $\mathbf{x}_{l} \in \Pi_{n}$ such that $r\left(A, B_{l}\right)=r\left(A, B_{l}, \mathbf{x}_{l}\right)$ for each $l \in \mathbb{N}$. Denote $t=\limsup r\left(A, B_{l}\right), s=\liminf r\left(A, B_{l}\right)$. Let $B_{l}=C_{l}+D_{l}, C_{l}, D_{l} \geq 0$, where supp $C_{l}=\operatorname{supp} B$ and supp $C_{l} \cap \operatorname{supp} D_{l}=\emptyset$. Clearly, $\lim _{l \rightarrow \infty} C_{l}=B$ and $\lim _{l \rightarrow \infty} D_{l}=0$. Fix $\varepsilon>0$. Then there exists $M(\varepsilon)$ such that $B \leq(1+\varepsilon) C_{l}$ for $l>M(\varepsilon)$. In particular, $B \leq(1+\varepsilon) B_{l}$ for $l>M(\varepsilon)$. Hence

$$
\frac{1}{1+\varepsilon} r\left(A, B_{l}, \mathbf{x}\right)=r\left(A,(1+\varepsilon) B_{l}, \mathbf{x}\right) \leq r(A, B, \mathbf{x}) .
$$

Therefore

$$
\frac{1}{1+\varepsilon} \rho\left(A, B_{l}\right)=\frac{1}{1+\varepsilon} \hat{\rho}\left(A, B_{l}\right)=\hat{\rho}\left(A,(1+\varepsilon) B_{l}\right) \leq \hat{\rho}(A, B) \text { for } l>M(\varepsilon) .
$$

As $\varepsilon>0$ was chose arbitrary it follows that $t \leq \hat{\rho}(A, B)$.

There exists a subsequence $1 \leq l_{1}<l_{2}<\cdots$ such that $\lim _{k \rightarrow \infty} \rho\left(A, B_{l_{k}}\right)=s$ and $\lim _{k \rightarrow \infty} \mathbf{x}_{l_{k}}=\mathbf{x} \in \Pi_{n}$. The inequality $A \mathbf{x}_{l_{k}} \leq r\left(A, B_{l_{k}}\right) B_{l_{k}} \mathbf{x}_{l_{k}}$ yield $A \mathbf{x} \leq s B \mathbf{x}$. (We may have that $s=\infty$.) Suppose first that $s<\infty$. Hence $s \geq \hat{\rho}(A, B)$. Combine that with the inequality $t \leq \hat{\rho}(A, B)$ to deduce that $s=t=\hat{\rho}(A, B)$. Assume second that $s=\infty$. Then $t=\infty$ and the inequality $t \leq \hat{\rho}(A, B)$ yields that $\hat{\rho}(A, B)=\infty$.

(5) Denote $t=\limsup r\left(A_{l}, B\right), s=\liminf r\left(A_{l}, B\right)$. Let $A_{l}=C_{l}+D_{l}, C_{l}, D_{l} \geq 0$, where supp $C_{l}=\operatorname{supp} A$ and supp $C_{l} \cap \operatorname{supp} D_{l}=\emptyset$. Clearly, $\lim _{l \rightarrow \infty} C_{l}=A$ and $\lim _{l \rightarrow \infty} D_{l}=0$. Fix $\varepsilon \in(0,1)$ in the rest of the proof. Then there exists $M(\varepsilon)$ such that $(1-\varepsilon) C_{l} \leq A \leq(1+\varepsilon) C_{l}$ for $l>M(\varepsilon)$. In particular, $A \leq(1+\varepsilon) A_{l}$ for $l>M(\varepsilon)$. Hence for $\mathbf{x} \gtrless \mathbf{0}$

$$
(1+\varepsilon) r\left(A_{l}, B, \mathbf{x}\right)=r\left((1+\varepsilon) A_{l}, B, \mathbf{x}\right) \geq r(A, B, \mathbf{x}) .
$$

Therefore

$$
(1+\varepsilon) \rho\left(A_{l}, B\right)=\rho\left((1+\varepsilon) A_{l}, B\right) \geq \rho(A, B) \text { for } l>M(\varepsilon) .
$$

As $\varepsilon>0$ was chosen arbitrary it follows that $s \geq \rho(A, B)$. As $B$ does not have zero row it follows that $\rho(A, B) \leq r\left(A, B, \mathbf{1}_{n}\right)<\infty$. Let $\mathbf{x}(\varepsilon) \in \Pi_{n}^{o}$ satisfy $A \mathbf{x}(\varepsilon) \leq(\rho(A, B)+\varepsilon) B \mathbf{x}(\varepsilon)$. As $B$ does not have a zero row it follows that $B \mathbf{x}(\varepsilon)>\mathbf{0}$. Since $\lim _{l \rightarrow \infty} D_{l}=0$ there exists $L(\varepsilon) \geq M(\varepsilon)$ such that $D_{l} \mathbf{x}(\varepsilon) \leq \varepsilon B \mathbf{x}(\varepsilon)$ for $l>L(\varepsilon)$. Then

$$
\begin{aligned}
& A_{l} \mathbf{x}(\varepsilon)=C_{l} \mathbf{x}(\varepsilon)+D_{l} \mathbf{x}(\varepsilon) \leq(1-\varepsilon)^{-1} A \mathbf{x}(\varepsilon)+\varepsilon B \mathbf{x}(\varepsilon) \leq \\
& \left((1-\varepsilon)^{-1}(\rho(A, B)+\varepsilon)+\varepsilon\right) B \mathbf{x}(\varepsilon) \text { for } l>L(\varepsilon)
\end{aligned}
$$


That is, $\rho\left(A_{l}, B\right) \leq\left((1-\varepsilon)^{-1}(\rho(A, B)+\varepsilon)\right.$ for $l>L(\varepsilon)$. Therefore $t \leq\left((1-\varepsilon)^{-1}(\rho(A, B)+\varepsilon)+\varepsilon\right)$. As $\varepsilon$ was an arbitrary number in the open interval $(0,1)$ it follows that $t \leq \rho(A, B)$. Combine that with the inequality $s \geq \rho(A, B)$ to deduce that $s=t=\rho(A, B)$.

\section{The Classical Case}

In this section we discuss the case where $m=n$ and $B$ is the identity matrix $I$. We first recall some basic results on directed graphs $\vec{G}=(V, \vec{E})$. Here $V$ is a finite set of vertices and $\vec{E} \subseteq V \times V$ is the set of diedges of $\vec{G}$. An ordered tuple $(v, w) \in \vec{E}$ is a diedge from $v$ to $w$. The diedge $(v, v)$ is called a loop. A dipath $\vec{P}$ in $\vec{G}$ is an ordered set of diedges $\left\{\left(v_{1}, v_{2}\right),\left(v_{2}, v_{3}\right), \ldots,\left(v_{p}, v_{p+1}\right)\right\}$ for a positive integer $p$. A dipath $\vec{P}$ is closed if $v_{p+1}=v_{1}$. The digraph $\vec{G}$ is called acyclic or diforest if $\vec{G}$ does not have a closed path. The digraph $\vec{G}$ is called strongly connected if for any two distinct vertices $v, w \in V$ there is a dipath in $\vec{G}$ from $v$ to $w$. (A digraph on one vertex with no diedge is strongly connected.)

For $W \subseteq V$ we define the induced subdigraph $\vec{G}(W)=(W, \vec{E}(W))$, where $\vec{E}(W)$ is the set of diedges in $\vec{E}$ that connects two vertices in $W$. Assume that $\vec{G}$ is not strongly connected. Then the subgraph $\vec{G}(W)$ is called strongly connected component of $\vec{G}$ if the subgraph $\vec{G}(W)$ is strongly connected but $\vec{G}(U)$ is not strongly connected for each $U$ that strictly contains $W$. Let $V=\cup_{i=1}^{k} V_{i}$ be the partition of $V$ corresponding to the strongly connected components of $\vec{G}$. That is $\vec{G}\left(V_{i}\right)$ for $i \in[k]$ are all strongly connected components of $\vec{G}$. The reduced digraph $\vec{G}_{\text {red }}=\left(V_{\text {red }}, \vec{E}_{\text {red }}\right)$ of $\vec{G}$ is defined as follows: First $V_{\text {red }}=\left\{\left\{V_{1}\right\}, \ldots,,\left\{V_{k}\right\}\right\}$. Second a diedge $\left(\left\{V_{i}\right\},\left\{V_{j}\right\}\right)$ is in $\vec{E}_{\text {red }}$ if $i \neq j$ and there is a diedge in $\vec{E}$ from $V_{i}$ to $V_{j}$. For a strongly connected digraph $\vec{G}$ we let $V_{\text {red }}=\{\{V\}\}$ and $\vec{E}_{\text {red }}=\emptyset$. It is straightforward to show that $\vec{G}_{\text {red }}$ is acyclic. With each digraph we associated an undirected graph $G=(V, E)$, where undirected edge $\{i, j\}$ is in $E$ if either $(i, j)$ or $(j, i)$ in $\vec{E}$. Then $G$ is a union of its connected components $G\left(W_{j}\right), j \in[c]$, where each $V_{i}$ is a subset of some $W_{j}$. Clearly, each $W_{j}$ is union of some subsets $V_{1}, \ldots, V_{k}$. The subset $W_{j}$ induces a subdigraph $\vec{G}\left(W_{j}\right)$. Observe that $\vec{G}\left(W_{j}\right)$ induces a reduced digraph $\vec{G}\left(W_{j}\right)_{\text {red }}$, which is a subdigraph of the reduced graph of $\vec{G}_{r e d}$. The subdigraph $\vec{G}\left(W_{j}\right)_{\text {red }}$ is called a ditree of $\vec{G}_{r e d}$. A vertex $\left\{V_{p}\right\}$ is called a source of $\vec{G}_{r e d}$ if there is no $\left\{V_{q}\right\}$ such that there is a diedge in $\vec{G}_{r e d}$ from $\left\{V_{q}\right\}$ to $\left\{V_{p}\right\}$. A vertex $\left\{V_{p}\right\}$ is called a sink of $\vec{G}_{\text {red }}$ if there is no $\left\{V_{q}\right\}$ such that there is a diedge in $\vec{G}_{r e d}$ from $\left\{V_{p}\right\}$ to $\left\{V_{q}\right\}$. Clearly, each ditree $\vec{G}\left(W_{j}\right)_{\text {red }}$ contains at least one source $\left\{V_{p}\right\}$ and one $\operatorname{sink}\left\{V_{q}\right\}$. Denote by $\mathcal{R}(\vec{G}) \subseteq[k]$ all indices $p \in[k]$ such that $\left\{V_{p}\right\}$ is a source.

Let $A=\left[a_{i j}\right] \in \mathbb{R}_{+}^{m \times m}$. One associates with $A$ the digraph $\vec{G}(A)=([m], \vec{E}(A))$. A diedge $(i, j)$ is in $\vec{E}(A)$ if and only if $a_{i j}>0$. $A$ is called irreducible if $\vec{G}(A)$ is strongly connected. Let $G(A)$ be the induced undirected graph. Then after renaming the indices $A$ is pemutationally similar to a block diagonal matrix $\operatorname{diag}\left(A_{1}, \ldots, A_{c}\right)$ if and only if $G(A)$ has $c$-connected components. Each $A_{j}$ corresponds to $\vec{G}\left(W_{j}\right)$. For each nonzero subset $U \subseteq[m]$ denote by $A[U]$ the submatrix $\left[a_{i j}\right]_{i, j \in U}$. Each $A_{j}$ is can be assumed to be in the Frobenius normal form [12, Theorem 6.4.4]. It is a block upper triangular form, where each diagonal block is an irreducible matrix $A\left[V_{i}\right]$, where $V_{i} \subseteq W_{j}$. (The order of the diagonal blocks depends on the labeling of the vertices of the ditree $\vec{G}\left(W_{j}\right)_{\text {red }}$. One such labeling is given in [12, Theorem 6.4.4].) We assume that the top diagonal block of $A\left[W_{j}\right]$ is $A\left[V_{p}\right]$ where $\left\{V_{p}\right\}$ is a source in the ditree $\vec{G}\left(W_{j}\right)_{\text {red }}$ and the bottom diagonal block is $A\left[V_{q}\right]$ where $\left\{V_{q}\right\}$ is a sink in $\vec{G}\left(W_{j}\right)_{\text {red }}$. Furthermore, every source $\left\{V_{p}\right\}$ in can be chosen to be the top diagonal block in $A\left[W_{j}\right]$. In particular

$$
\rho(A)=\max _{i \in[k]} \rho\left(A\left[V_{i}\right]\right), \quad \rho\left(A\left[W_{j}\right]\right)=\max _{V_{i} \subseteq W_{j}} \rho\left(A\left[V_{i}\right]\right) .
$$

We first bring the well known result due to Wielandt 39.

Lemma 3.1. Let $A \in \mathbb{R}_{+}^{m \times m}$ be an irreducible matrix. Then $\rho(A, I)=\hat{\rho}(A, I)=\rho(A)$. Furthermore, in characterization (2) equality holds if and only if $\mathrm{x}$ is the PF-eigenvector of $A$.

Proof. The equality $\rho(A, I)=\rho(A)$, i.e., the characterization (2) was proved by Wielandt [39]. Wielandt also showed that equality in (2) holds if and only if $\mathbf{x}$ is the PF-eigenvector of $A$. The equality $\rho(A, I)=\hat{\rho}(A, I)$ follows from the following observation: Assume that $\mathbf{x}=\left(x_{1}, \ldots, x_{m}\right)^{\top} \geqslant 0$ and $1 \leq|\operatorname{supp} \mathbf{x}|<m$. Then $r(A, I, \mathbf{x})=\infty$. Indeed, let $\mathcal{I}=[m] \backslash \operatorname{supp} \mathbf{x}$. As $\vec{G}(A)$ is irreducible there exists $(i, j) \in \vec{E}(A)$ such that $i \in \mathcal{I}$ and $j \in \operatorname{supp} \mathbf{x}$. Hence $a_{i j}>0$. Therefore $x_{i}=0$ and $(A \mathbf{x})_{i}>0$, which yield that $\frac{(A \mathbf{x})_{i}}{x_{i}}=\infty$. 
Theorem 3.2. Assume that $A \in \mathbb{R}_{+}^{m \times m}$. Then

(1) $\rho(A, I)=\rho(A)$, and $A \mathbf{y}=\rho(A) \mathbf{y}$ for some $\mathbf{y} \gtrless \mathbf{0}$.

(2) There exists $\mathbf{x}=\left(x_{1}, \ldots, x_{m}\right)^{\top}>\mathbf{0}$ such that $\rho(A)=\max _{i \in[m]} \frac{(A \mathbf{x})_{i}}{x_{i}}$ if and only if the following condition hold: Let $\vec{G}_{\text {red }}$ be the induced reduced graph by $A$, with the set of vertices $\left\{\left\{V_{1}\right\}, \ldots,\left\{V_{k}\right\}\right\}$. The equality $\rho\left(A\left[V_{j}\right]\right)=\rho(A)$ implies that $\left\{V_{j}\right\}$ is a sink of $\vec{G}_{r e d}$.

(3) The vector $\mathbf{x} \in \Pi_{m}^{o}$ such that $\rho(A)=\max _{i \in[m]} \frac{(A \mathbf{x})_{i}}{x_{i}}$ is unique if and only if $A$ is irreducible.

(4) $\hat{\rho}(A, I)=\min _{i \in \mathcal{R}(\vec{G}(A))} \rho\left(A\left[V_{i}\right]\right)$ and $A \mathbf{w}=\hat{\rho}(A, I) \mathbf{w}$ for some $\mathbf{w} \gtrless \mathbf{0}$.

Proof. (1) Recall the Perron's result that claims that a positive square matrix $C$ has a positive eigenvector u corresponding to $\rho(C)$. Assume that $B \in \mathbb{R}_{+}^{m \times m}$. Let $J \in \mathbb{R}_{+}^{m \times m}$ be a matrix whose all entries are 1. Set $B_{l}=B+\frac{1}{l} J$ for each positive integer $l$. Perron's theorem implies the existence of $\mathbf{u}_{l} \in \Pi_{m}^{o}$ such that $B_{l} \mathbf{u}_{l}=\rho\left(B_{l}\right) \mathbf{u}_{l}$. Since $\Pi_{m}$ is compact there is a subsequence of $\left\{\mathbf{u}_{l}\right\}$ which converges to $\mathbf{u} \in \Pi_{m}$. Clearly, $\lim _{l \rightarrow \infty} \rho\left(B_{l}\right)=\rho(B)$. Hence $B \mathbf{u}=\rho(B) \mathbf{u}$. Choose $B=A$. Then $r\left(A, I, \mathbf{u}_{l}\right)<\rho\left(A_{l}, I . \mathbf{u}_{l}\right)=\rho\left(A_{l}\right)$. Therefore $\rho(A, I) \leq \rho(A)$. Furthermore $A$ has a nonnegative eigenvector $\mathbf{y}$ corresponding to $A$. Let $\mathbf{x}>\mathbf{0}$. We show that $r(A, I, \mathbf{x}) \geq \rho(A)$. Assume that $A^{\top} \mathbf{u}=\rho(A) \mathbf{u}$, where $\mathbf{u} \gtrless \mathbf{0}$. Then $\mathbf{u}^{\top}(r(A, I, \mathbf{x}) \mathbf{x}) \geq \mathbf{u}^{\top} A \mathbf{x}=$ $\rho(A) \mathbf{u}^{\top} \mathbf{x}$. As $\mathbf{x}>\mathbf{0}$ we deduce that $\mathbf{u}^{\top} \mathbf{x}>0$. Hence $r(A, I) \geq \rho(A)$. (This argument is in 8 .) Hence $\rho(A, I) \geq \rho(A)$ and $\rho(A, I)=\rho(A)$.

(2) Assume that there exists $\mathbf{x}=\left(x_{1}, \ldots, x_{m}\right)^{\top}>\mathbf{0}$ such that

$\rho(A)=\max _{i \in[m]} \frac{(A \mathbf{x})_{i}}{x_{i}}$. Let $\left\{\left\{V_{1}\right\}, \ldots,\left\{V_{k}\right\}\right\}$ be the vertices of the reduced graph induced by $A$. Suppose that $\rho(A)=\rho\left(A\left[V_{j}\right]\right)$. Let $\mathbf{z}=\left(z_{1}, \ldots, z_{l}\right)^{\top} \in \mathbb{R}_{+}^{\left|V_{j}\right|}$ be the subvector of $\mathbf{x}$ restricted to the set $V_{j}$. Hence

$$
\rho(A)=\rho\left(A\left[V_{j}\right]\right) \leq \max _{i \in\left[\left|V_{j}\right|\right]} \frac{\left(A\left[V_{j}\right] \mathbf{z}\right)_{i}}{z_{i}} \leq \max _{i \in V_{j}} \frac{(A \mathbf{x})_{i}}{x_{i}} \leq \rho(A) .
$$

Therefore all the inequalities are equalities. The first equality and Lemma 3.1 yield that $A\left[V_{j}\right] \mathbf{z}=\rho\left(A\left[V_{j}\right]\right) \mathbf{z}$. The second equality yields that $V_{j}$ is a sink.

Vice versa assume that $\rho\left(A\left[V_{j}\right]\right)=\rho(A)$ if and only if $\left\{V_{j}\right\}$ is a sink of $\vec{G}_{r e d}$. We now show that there exists $\mathbf{x}=\left(x_{1}, \ldots, x_{m}\right)^{\top}>\mathbf{0}$ such that equality holds in (2). Without loss of generality we may assume that $\rho(A)>0$. (Otherwise $A=0$.) Let $\mathcal{C}_{0}\left(\vec{G}_{\text {red }}\right) \subseteq\left\{\left\{V_{1}\right\}, \ldots,\left\{V_{k}\right\}\right\}$ be the set of the sink vertices in $\vec{G}_{r e d}$. Assume that $\left\{V_{j}\right\}$ is a sink. Then the restriction of $\mathbf{x}$ to $V_{j}$ is the PF-eigenvector $\mathbf{x}_{j}>\mathbf{0}$ of $A\left[V_{j}\right]$. If $\mathcal{C}_{0}\left(\vec{G}_{r e d}\right)=\left\{\left\{V_{1}\right\}, \ldots,\left\{V_{k}\right\}\right\}$ we easily deduce that equality holds in (2) for this $\mathbf{x}$. If not let us consider the subsets $\mathcal{C}_{l}\left(\vec{G}_{\text {red }}\right) \subset\left\{\left\{V_{1}\right\}, \ldots,\left\{V_{k}\right\}\right\}$ for $l=0,1, \ldots, p$, which is a partition of $\left\{\left\{V_{1}\right\}, \ldots,\left\{V_{k}\right\}\right\}$, defined as follows. For $l \geq 1$ the set $\mathcal{C}_{l}\left(\vec{G}_{\text {red }}\right) \subset\left\{\left\{V_{1}\right\}, \ldots,\left\{V_{k}\right\}\right\}$ consists of vertices in $\vec{G}_{\text {red }}$ with the maximal path length $l$ in the digraph $\vec{G}_{\text {red }}$ starting at these vertices. Thus the diedges in $\vec{G}_{\text {red }}$ from $\mathcal{C}_{l}\left(\vec{G}_{r e d}\right)$ go only to $\mathcal{C}_{r}\left(\vec{G}_{r e d}\right)$ for $r=0,1, \ldots, l-1$, and each $\left\{V_{j}\right\} \in \mathcal{C}_{l}\left(\vec{G}_{r e d}\right)$ has at least one diedge. (This corresponds to the Frobenius normal form given in [12, Theorem 6.4.4].) Suppose that we already determined the restriction of $\mathbf{x}$ to $\left\{V_{q}\right\} \in \mathcal{C}_{r}\left(\vec{G}_{r e d}\right)$ for $r \leq l-1$, which is denoted by $\mathbf{x}_{q}$, where $\mathbf{x}_{q}>0$. We now show how to determine $\mathbf{x}_{j}$ for each $\left\{V_{j}\right\} \in \mathcal{C}_{l}\left(\vec{G}_{r e d}\right)$. Recall that $\rho\left(A\left[V_{j}\right]\right)<\rho(A)$. Choose $t_{j} \in\left(\rho\left(A\left[V_{j}\right]\right), \rho(A)\right]$. We now determine $\mathbf{x}_{j}$ by the condition $(A \mathbf{x})\left[V_{j}\right]=t_{j} \mathbf{x}_{j}$. Denote by $A\left[V_{j}, V_{q}\right]$ the restriction of $A$ to rows in the set $V_{j}$ and columns in the set in $V_{q}$. Then the above condition is equivalent to

$$
\left(t_{j} I_{V_{j}}-A\left[V_{j}\right]\right) \mathbf{x}_{j}=\sum_{V_{q} \in \cup_{r=0}^{l-1} \mathcal{C}_{r}\left(\vec{G}_{r e d}\right)} A\left[V_{j}, V_{q}\right] \mathbf{x}_{q}
$$

Since all $\mathbf{x}_{q}>\mathbf{0}$ for $V_{q} \in \cup_{r=0}^{l-1} \mathcal{C}_{r}\left(\vec{G}_{r e d}\right)$ and at least one of $A\left[V_{j}, V_{q}\right]$ is a nonzero nonnegative matrix it follows that the right hand side in the above equality is a nonzero nonnegative vector. As $t_{j}>\rho\left(A\left(V_{j}\right)\right)$ and $A\left[V_{j}\right]$ is irreducible it follows that $\left(t_{j} I_{V_{j}}-A\left[V_{j}\right]\right)^{-1}$ is a positive matrix [12, Lemma 6.4.3]. Therefore

$$
\mathbf{x}_{j}=\left(t_{j} I_{V_{j}}-A\left(V_{j}\right)\right)^{-1} \sum_{V_{q} \in \cup_{r=0}^{l-1} \mathcal{C}_{r}\left(\vec{G}_{r e d}\right)} A\left[V_{j}, V_{q}\right] \mathbf{x}_{q}>\mathbf{0} .
$$

This shows that the constructed $\mathbf{x}$ is a positive vector. It is left to show that $\rho(A)=\max _{i \in[m]} \frac{(A \mathbf{x})_{i}}{x_{i}}$. Assume that $i \in V_{j}$. Then our construction gives that $\frac{(A \mathbf{x})_{i}}{x_{i}}=t_{j} \leq \rho(A)$. For a sink $\left\{V_{j}\right\}$ such that $\rho\left(A\left(V_{j}\right)\right)=\rho(A)$ we have that $t_{j}=\rho(A)$. Hence $\rho(A)=\max _{i \in[m]} \frac{(A \mathbf{x})_{i}}{x_{i}}$. 
(3) The proof of 2 shows that $\mathbf{x} \in \Pi_{m}^{o}$ is unique if and only if $A$ is an irreducible matrix.

(4) Clearly, it is enough to show the equality $\hat{\rho}(A, I)=\min _{i \in \mathcal{R}(\vec{G}(A))} \rho\left(A\left[V_{i}\right]\right)$ in the case where $\vec{G}(A)_{\text {red }}$ is a ditree, that is, $G(A)$ is a connected graph. Let $\left\{V_{i}\right\}$ be a source of $\vec{G}(A)_{\text {red }}$. So we can choose a Frobenius normal form so that the irreducible matrix $A\left[V_{i}\right]$ appears in the first diagonal block of the Frobenius normal form $F$ of $A$. Let $A\left[V_{i}\right] \mathbf{z}=\rho\left(A\left[V_{i}\right]\right) \mathbf{z}$ where $\mathbf{z}>0$. Extend $\mathbf{z}$ to $\mathbb{R}_{+}^{m}$ by addind zero entries for indices $\left\{\left|V_{i}\right|+1, \ldots, m\right\}$ to obtain the vector $\mathbf{v} \in \mathbb{R}_{+}^{m}$. Then $F \mathbf{v}=\rho\left(A\left[U_{i}\right]\right) \mathbf{v}$. Rename the name of the indices to deduce that $A \mathbf{w}=\rho\left(A\left[U_{i}\right]\right) \mathbf{w}$. Hence $r(A, I, \mathbf{w})=\rho\left(A\left[U_{i}\right]\right)$ and $\hat{\rho}(A, I) \leq \rho\left(A\left[U_{i}\right]\right)$. This shows that $\hat{\rho}(A, I) \leq \alpha=\min _{i \in \mathcal{R}(\vec{G}(A))} \rho\left(A\left[V_{i}\right]\right)$.

It is left to show the reverse inequality $r(A, I, \mathbf{x}) \geq \alpha$ for each $\mathbf{x} \geq \mathbf{0}$ such that $r(A, I, \mathbf{x})<\infty$. Suppose first that $\mathbf{x}>\mathbf{0}$. The above arguments yield that $r(A, I, \mathbf{x}) \geq \rho(A) \geq \alpha$. Assume now that supp $\mathbf{x}$ is a strict subset of $[m]$. Let $\mathcal{I} \subseteq[k]$ the the set of all $i \in[k]$ such that $\operatorname{supp} \mathbf{x} \cap V_{i} \neq \emptyset$. We claim that for each $i \in \mathcal{I}$ we have the equality supp $\mathbf{x} \cap V_{i}=V_{i}$. Indeed assume that $\operatorname{supp} \mathbf{x} \cap V_{i}$ is a strict subset of $V_{i}$. Let $\mathbf{x}_{i}=\mathbf{x}\left[V_{i}\right] \in \mathbb{R}_{+}^{\left|V_{i}\right|}$ be the restriction of $\mathbf{x}$ to $V_{i}$. Thus $\mathbf{x}_{i} \gtrless 0$. Since $A\left[V_{i}\right]$ is an irreducible matrix, the proof of Lemma 3.1 yield that there exists $p \in V_{i} \backslash \operatorname{supp} \mathbf{x}$ such that $\left(A\left[V_{i}\right] \mathbf{x}_{i}\right)_{p}>0$. As $(A \mathbf{x})_{p} \geq\left(A\left[V_{i}\right] \mathbf{x}_{i}\right)_{p}$ it follows that $\frac{(A \mathbf{x})_{p}}{x_{p}}=\frac{t}{0}=\infty$. So $r(A, I, \mathbf{x})=\infty$ contrary to our assumption. Assume that $\left\{V_{i}\right\}$ is a source in $\vec{G}(A)_{\text {red }}$. Clearly $r(A, I, \mathbf{x}) \geq r\left(A\left[V_{i}\right], I, \mathbf{x}_{i}\right)$. Lemma 3.1 yields that $r\left(A\left[V_{i}\right], I, \mathbf{x}_{i}\right) \geq \rho\left(A\left[V_{i}\right]\right)$. Hence $r\left(A\left[V_{i}\right], I, \mathbf{x}_{i}\right) \geq \alpha$. Assume that $\left\{V_{i}\right\}$ is not a source. Hence there is $j \in[k]$ such that $(j, i) \in \vec{E}\left(\vec{G}(A)_{\text {red }}\right)$. We claim that supp $\mathbf{x} \cap V_{j}=V_{j}$. Suppose not. So supp $\mathbf{x} \cap V_{j}=\emptyset$. From the definition of the reduced graph $\vec{G}(A)_{\text {red }}$ it follows there is $p \in V_{j}$ and $q \in V_{i}$ such that $a_{p q}>0$. Hence $(A \mathbf{x})_{p}>0$ and $x_{p}=0$. Thus $r(A, I, \mathbf{x})=\infty$ which contradicts our assumption. Thus supp $\mathbf{x} \cap V_{j}=V_{j}$. Repeating this argument a number of steps we deduce that there is a source vertex $\left\{V_{l}\right\}$ in $\vec{G}(A)_{\text {red }}$ such that supp $\mathbf{x} \cap V_{l}=V_{l}$. The previous arguments yield that $r(A, I, \mathbf{x}) \geq \rho\left(A\left[V_{l}\right]\right) \geq \alpha$.

We remark that part 1 of this theorem is well known, part 2 is close to [12, Theorem 6.4.5], part 3 is perhaps known, and part 4 seems to be new.

Example 3.3. Let $A=\left[\begin{array}{ccc}a & 1 & 1 \\ 0 & 1 & 1 \\ 0 & 0 & 1+a\end{array}\right]$, where $a \in(0,1]$, and $B=I_{3}$. Then

(1) $\rho\left(A, I_{3}\right)=\rho(A)=1+a, \mathbf{x}=\left(1+2 a^{-1}, 2 a^{-1}, 1\right)^{\top}$ is optimal and $\mathbf{y}=(1,1,0)^{\top}$ is minimal optimal.

(2) $\hat{\rho}(A, I)=a$ and $\mathbf{y}=(1,0,0)^{\top}$ is the unique weak minimal vector in $\Pi_{3}$.

Proof. (1) It is straightford to show that $r(A, I, \mathbf{x})=(1+a)$, hence $\mathbf{x}$ is optimal. Take $\mathbf{y}(t)=(1,1, t)^{\top}$ for $t>0$. Then $r(A, B, \mathbf{y}(t))=1+a+t$. Let $t \rightarrow 0$ to deduce that $\mathbf{y}$ is optimal. Suppose to the contrary that $\mathbf{y}$ is not minimal optimal. Thus either $\mathbf{u}=(1,0,0)^{\top}$ or $\mathbf{v}=(0,1,0)^{\top}$ is minimal optimal. But $r(A, I, \mathbf{u})=a$ and $r(A, I, \mathbf{v})=\infty$. Contradiction.

(2) Part 4 of Theorem 3.2 yields that $\hat{\rho}(A, I)=a$. It is straightforward to show that $\mathbf{y}=(1,0,0)^{\top}$ is the unique weak minimal vector in $\Pi_{3}$.

\section{Polynomial approximation of $\rho(A, B)$ And $\hat{\rho}(A, B)$}

We first recall the fundamental result that a feasibility of a system of linear inequalities and minimization of a linear function are polynomial. For simplicity we state a variant of this fact in the following setting that we need.

Lemma 4.1. Let $C \in \mathbb{Q}^{m \times n}, \mathbf{b}=\left(b_{1}, \ldots, b_{m}\right)^{\top} \in \mathbb{Q}^{m}, \mathbf{c}=\left(c_{1}, \ldots, c_{n}\right)^{\top} \in \mathbb{Q}^{n}$. Then one can find in polynomial time in $\langle C\rangle+\langle\mathbf{b}\rangle$ if the the following polytope is empty or not

$$
C \mathbf{x} \geq \mathbf{b}, \quad \mathbf{x}=\left(x_{1}, \ldots, x_{n}\right)^{\top} \geq \mathbf{0}, \sum_{i=1}^{n} x_{i}=1 .
$$

Furthermore, if the above polytope is nonempty then the minimum of the linear function $\mathbf{c}^{\top} \mathbf{x}$ over this polytope can be found in polynomial time.

This result is well known, see for example [26, 19. To be precise, [26, Corollary 2.3.7] assumes for simplicity that the system (13) is solvable, and then one can find the maximum or minimum of $\mathbf{c}^{\top} \mathbf{x}$, where $\mathbf{c} \in \mathbb{Q}^{n}$ is 
given and $\mathrm{x}$ satisfies (13). To apply [26. Corollary 2.3.7] for solvable system we consider the following linear programing problems $L P_{j}$ for $j \in[m]$. Let $\mathbf{c}_{j}^{\top}$ be the $j-t h$ row of $C$. Let $C_{j} \in \mathbb{Q}^{(j-1) \times n}, \mathbf{b}_{j} \in \mathbb{Q}^{j-1}$ be the matrix and the column obtained from $C$ and $\mathbf{b}$ by deleting the $m-j+1$ rows $j, \ldots, m$ respectively. Assume that the system

$$
C_{j} \mathbf{x} \geq \mathbf{b}_{j}, \quad \mathbf{x}=\left(x_{1}, \ldots, x_{n}\right)^{\top} \geq \mathbf{0}, \sum_{i=1}^{n} x_{i}=1
$$

is solvable for $j+1=\ell \in[n]$. (This is trivially true for $\ell=1$.) Now consider the maximum problem $\max \mathbf{c}_{\ell}^{\top} \mathbf{x}$ over the set given by (14) for $j=\ell$. Assume that the maximum is achieved at $\mathbf{x}_{\ell}$. Then the polytope given by (14) for $j=\ell+1$ is nonempty if and only if $\mathbf{c}^{\top} \mathbf{x}_{\ell} \geq b_{\ell}$. Hence by running at most $m$ linear programming problems we can determine in polynomial time if the system (13) is feasible or not.

Theorem 4.2. Let $A, B \in \mathbb{Q}_{+}^{m \times n} \backslash\{0\}$. Assume that $\rho(A, B)<\infty$. Then for any $\varepsilon \in(0,1) \cap \mathbb{Q}$ one of the following statements can be verified in polynomial time in $\langle A\rangle+\langle B\rangle+\langle\varepsilon\rangle$ :

(1) The Collatz-Wielandt quotient satisfies $\rho(A, B)<\varepsilon$.

(2) The Collatz-Wielandt quotient is positive and one can find $\tilde{\rho}(A, B) \in \mathbb{Q}_{+} \backslash\{0\}$, such that

$$
\tilde{\rho}(A, B) \leq \rho(A, B) \leq(1+\varepsilon) \tilde{\rho}(A, B) .
$$

Proof. Let $t_{0}=r\left(A, B, \mathbf{1}_{n}\right)<\infty$ and $N=\left\lceil\log _{2} \varepsilon^{-1}\right\rceil+1$. Set $k=1, t_{k}=\frac{1}{2} t_{k-1}$ and $C=t_{k} B-A$. Consider the system (13) with $\mathbf{b}=\mathbf{0}$. Assume first that this system is solvable. Let $\mu_{i, k}$ be the maximum of $x_{i}$ for the system (13) with $\mathbf{b}=\mathbf{0}$ for $i \in[n]$. Assume first that $\mu_{i, k}>0$ for each $i \in[n]$. We claim that $\rho(A, B) \leq t_{k}$. Indeed, assume that $\mu_{i, k}=x_{i, i, k}$ where $\mathbf{x}_{i, k}=\left(x_{1, i, k}, \ldots, x_{n, i, k}\right)^{\top} \in \mathbb{Q}^{n}$ satisfies the system (13) with $\mathbf{b}=\mathbf{0}$. Set $\mathbf{x}=\frac{1}{n} \sum_{i=1}^{n} \mathbf{x}_{i, k}$ to deduce that $r(A, B, \mathbf{x}) \leq t_{k}$. Assume that for $k=2, \ldots, N$ we have the inequality $\rho(A, B) \leq t_{k}$. Then $\rho(A, B) \leq t_{N}<\varepsilon$ and we showed the condition (1).

Suppose now that for the smallest value $k \in[N]$ one of the following conditions hold: Either the system (13) with $C=t_{k} B-A$ and $\mathbf{b}=\mathbf{0}$ is not solvable or $\mu_{i, k}=0$ for some $i \in[n]$. Then $0<t_{k} \leq \rho(A, B)$. Set $l=0$ $f_{l}=t_{k}, g_{l}=t_{k-1}$. Then $f_{l} \leq \rho(A, B) \leq g_{l}$. If $\frac{g_{l}-f_{l}}{f_{l}} \leq \varepsilon$ then $\tilde{\rho}(A, B)=f_{l}$. If not set $M=\left\lceil\log _{4 / 3} \frac{g_{l}-f_{0}}{f_{0} \varepsilon}\right\rceil$ and apply now the bisection algorithm: Let $h_{l}=\frac{f_{l}+g_{l}}{2}$ and $C=h_{l} B-A$. Consider the system (13) with $\mathbf{b}=\mathbf{0}$. Assume first that this system is solvable. Let $\mu_{i, l}$ be the minimum of $x_{i}$ for the system (13) with $\mathbf{b}=\mathbf{0}$ for $i \in[n]$. Suppose that $\mu_{i, l}>0$ for each $i$. Then $f_{l+1}=f_{l}, g_{l+1}=\frac{f_{l}+g_{l}}{2}$. If $\mu_{i, l}=0$ for some $i$ or the system (13) with $\mathbf{b}=\mathbf{0}$ is not solvable set $f_{l+1}=\frac{f_{l}+g_{l}}{2}, g_{l+1}=g_{l}$. Clearly $f_{l+1} \leq \rho(A, B) \leq g_{l+1}$. Continue this bisection to $l=M$. Then $\tilde{\rho}(A, B)=f_{M}$ and the condition (2) holds.

We now discuss briefly an approximation to $\hat{\rho}(A, B)$. First use use Algorithm 2.4 to determine if $\hat{\rho}(A, B)<$ $\infty$. Assume that $\hat{\rho}(A, B)<\infty$. Next assume that $\hat{\rho}(A, B)>0$, that is, the condition 7 of Lemma 2.1 holds. Then the right hand side of (12) as a positive lower bound for $\hat{\rho}(A, B)$. Use Algorithm 2.4 to find a nonempty subset $S \subseteq[n]$ such that $r\left(A, B, \mathbf{1}_{S}\right)<\infty$. Thus $r\left(A, B, \mathbf{1}_{S}\right)$ is an upper bound on $\hat{\rho}(A, B)$. Next apply a simplified version of the bisection algorithm used in the proof of Theorem 4.2. (without considering the minimum problem), to deduce:

Proposition 4.3. Let $A, B \in \mathbb{Q}_{+}^{m \times n} \backslash\{0\}$. Assume that $0<\hat{\rho}(A, B)<\infty$. Then for any $\varepsilon \in(0,1) \cap \mathbb{Q}$ one can find $\bar{\rho}(A, B) \in \mathbb{Q}_{+} \backslash\{0\}$ in polynomial time in $\langle A\rangle+\langle B\rangle+\langle\varepsilon\rangle$, such that

$$
\bar{\rho}(A, B) \leq \hat{\rho}(A, B) \leq(1+\varepsilon) \bar{\rho}(A, B) .
$$

\section{Minimal optimal solutions}

We first discuss weak optimal solutions which are easier to characterize.

Theorem 5.1. Let $m, n$ be positive integers. Assume that $A=\left[a_{i j}\right], B=\left[b_{i j}\right] \in \mathbb{R}_{+}^{m \times n}$. Suppose that $\hat{\rho}(A, B)<\infty$.

(1) Assume that $\mathbf{y} \in \mathbb{R}_{+}^{n} \backslash\{\mathbf{0}\}$ is weak optimal. Then at least one coordinate of $(A-\hat{\rho}(A, B) B) \mathbf{y}$ is zero.

(2) Assume that there exists a weak optimal vector $\mathbf{y} \in \mathbb{R}_{+}^{n}$ with $\ell$ positive coordinates. Let $A^{\prime}, B^{\prime} \in \mathbb{R}^{m \times \ell}$ be the submatrices of $A$ and $B$ respectively induced by the positive coordinates of $\mathbf{y}$. If $\ell \geq m$ then $\operatorname{rank}\left(A^{\prime}-\hat{\rho}(A, B) B^{\prime}\right)<m$.

(3) A minimal weak optimal $\mathbf{y}$ has at most $m$ positive coordinates. 
(4) Let $\mathbf{y}$ be a minimal weak optimal with $m$ positive coordinates. Then $\mathbf{y}$ is a weak GPF-vector. Furthermore $\operatorname{rank}\left(A^{\prime}-\hat{\rho}(A, B) B^{\prime}\right)=m-1$.

(5) Let $\mathbf{y}^{\prime}$ be a minimal weak optimal with $\ell<m$ positive coordinates. Then there exists a minimal weak optimal $\mathbf{y}$, satisfying supp $\mathbf{y}=\operatorname{supp} \mathbf{y}^{\prime}$ with the following property: Let $\mathcal{K}=\left\{k \in[m],(A \mathbf{y})_{k}=\right.$ $\left.\hat{\rho}(A, B)(B \mathbf{y})_{k}\right\}$. Then $|\mathcal{K}| \geq \ell$.

Proof. (1) Let $D(t)=A-t B$ and $t_{0}=\hat{\rho}(A, B)$. As $r(A, B, \mathbf{y})=t_{0}$ it follows that $C\left(t_{0}\right) \mathbf{y} \leq \mathbf{0}$. Suppose to the contrary that $C\left(t_{0}\right) \mathbf{y}<\mathbf{0}$. Then $t_{0}>0$. Furthermore, there exists $0 \leq t_{1}<t_{0}$ such that $C\left(t_{1}\right) \mathbf{y} \leq \mathbf{0}$. Hence $r(A, B, \mathbf{y}) \leq t_{1}<t_{0}$ contrary to our assumption.

(2) Assume that $\mathbf{y}$ is weak optimal vector which has $\ell$ positive coordinates. Let $A^{\prime}, B^{\prime} \in \mathbb{R}^{m \times \ell}$ be defined as in the theorem. Assume that $\mathbf{0}<\mathbf{z} \in \mathbb{R}^{\ell}$ is the subvector of $\mathbf{y}$ induced by its positive coordinates. Let $C(t)=A^{\prime}-t B^{\prime}$ and $t_{0}=\hat{\rho}(A, B)$. Then $C\left(t_{0}\right) \mathbf{z}=-\mathbf{w}, \mathbf{w} \in \mathbb{R}_{+}^{m}$. Assume $\ell \geq m$ and $\operatorname{rank} C\left(t_{0}\right)=m$. Then there exists a $m \times m$ submatrix of $C\left(t_{0}\right)$ which is nonsingular. By permuting the columns of $C\left(t_{0}\right)$ we can assume the following. Let $A^{\prime}=\left[A_{1} A_{2}\right], B^{\prime}=\left[\begin{array}{ll}B_{1} & B_{2}\end{array}\right]$ where $A_{1}, B_{1} \in \mathbb{R}^{m \times m}$ and $\operatorname{det}\left(A_{1}-t_{0} B_{1}\right) \neq 0$. Denote $C_{1}(t)=A_{1}-t B_{1}, C_{2}(t)=A_{2}-t C_{2}$. Assume that $\mathbf{z}^{\top}=\left(\mathbf{u}^{\top}, \mathbf{v}^{\top}\right), \mathbf{0}<\mathbf{u} \in \mathbb{R}^{m}, \mathbf{0}<\mathbf{v} \in \mathbb{R}^{\ell-m}$. Thus $C_{1}\left(t_{0}\right) \mathbf{u}=-\left(C_{2}\left(t_{0}\right) \mathbf{v}+\mathbf{w}\right)$. Since $\operatorname{det} C_{1}\left(t_{0}\right) \neq 0$, there exists $\varepsilon>0$ such that $\operatorname{det} C_{1}(t) \neq 0$ for $\left|t-t_{0}\right|<\varepsilon$. Observe that $\mathbf{u}=-C_{1}\left(t_{0}\right)^{-1}\left(C_{2}\left(t_{0}\right) \mathbf{v}+\mathbf{w}\right)>\mathbf{0}$. Let $\mathbf{u}(t)=-C_{1}(t)^{-1}\left(C_{2}(t) \mathbf{v}+\mathbf{w}\right)$ for $t \in\left(t_{0}-\varepsilon, t_{0}+\varepsilon\right)$. Then $\mathbf{u}(t)$ is continuous in the interval $\left(t_{0}-\varepsilon, t_{0}+\varepsilon\right)$. Hence there exists $\varepsilon_{1} \in(0, \varepsilon)$ such that $\mathbf{u}(t)>\mathbf{0}$ for $\left|t-t_{0}\right| \leq \varepsilon_{1}$. Set for $t_{1}=t_{0}-\varepsilon_{1}$ and $\mathbf{z}^{\prime}=\left(\mathbf{u}\left(t_{1}\right)^{\top}, \mathbf{v}^{\top}\right)^{\top}$. Thus $C\left(t_{1}\right) \mathbf{z}^{\prime}=-\mathbf{w}$, which implies that $r\left(A^{\prime}, B^{\prime}, \mathbf{z}^{\prime}\right) \leq t_{1}<\hat{\rho}\left(A^{\prime}, B^{\prime}\right)=\hat{\rho}(A, B)$. This contradicts the definition of $\hat{\rho}\left(A^{\prime}, B^{\prime}\right)$. Hence rank $C\left(t_{0}\right)<m$. (3) Assume to the contrary that $\mathbf{y}$ is a minimal weak optimal solution with $\ell>m$ positive coordinates. Let $A^{\prime}, B^{\prime}, C(t), \mathbf{z}, \mathbf{w}, t_{0}$ be defined as in part 2 of the proof. We showed that rank $C\left(t_{0}\right)<m$. Hence $\operatorname{dim} \operatorname{ker} C\left(t_{0}\right) \geq 2$. Choose $\mathbf{x} \in \operatorname{ker} C\left(t_{0}\right) \backslash\{\mathbf{0}\}$ such that $\mathbf{x}$ has at least one negative coordinate and one positive coordinate. So $\mathbf{y}$ is not proportional to $\mathbf{x}$. Let $\mathbf{z}(s)=\mathbf{z}+s \mathbf{x}$ for $s \geq 0$. Note that $C\left(t_{0}\right) \mathbf{z}(s)=-\mathbf{w}$. Let $s_{0}>0$ be the biggest $s$ such that $\mathbf{z}(s) \geq \mathbf{0}$. Then $\mathbf{z}\left(s_{0}\right) \geqslant \mathbf{0}, \mathbf{z}\left(s_{0}\right)$ has at least one zero component and $C\left(t_{0}\right) \mathbf{z}\left(s_{0}\right)=-\mathbf{w}$. Thus $r\left(A^{\prime}, B^{\prime}, \mathbf{z}\left(s_{0}\right)\right) \leq t_{0}=\hat{\rho}\left(A^{\prime}, B^{\prime}\right)$. Hence $r\left(A^{\prime}, B^{\prime}, \mathbf{z}\left(s_{0}\right)\right)=t_{0}$, which contradicts the minimality of $\mathbf{y}$.

(4) Assume that $\mathbf{y}$ a minimal weak optimal with $m$ positive coordinates. Use the notations of parts 2 and 3 of the proof. We claim that $C\left(t_{0}\right) \mathbf{z}=0$. Suppose not. By part 2 rank $C\left(t_{0}\right)<m$. Let $\mathbf{x} \in \operatorname{ker} C\left(t_{0}\right) \backslash\{\mathbf{0}\}$. So $\mathbf{y}$ is not proportional to $\mathbf{x}$. By considering $\pm \mathbf{x}$ we may assume that $\mathbf{x}$ has at least one negative coordinate. Define $\mathbf{z}\left(s_{0}\right)$ as in part (3) to deduce that $\mathbf{y}$ is not minimal. So $C\left(t_{0}\right) \mathbf{z}=0$. Assume to the contrary that rank $C\left(t_{0}\right)<m-1$. Choose $\mathbf{x} \in \operatorname{ker} C\left(t_{0}\right)$ to have positive and negative coordinates. Then we conclude as above that $\mathbf{z}$ is not minimal.

(5) Let $\mathbf{y}^{\prime}$ be a minimal weak optimal with $\ell<m$ positive coordinates. Part 1 of the theorem yields that $C\left(t_{0}\right) \mathbf{y}^{\prime}$ has at least on zero coordinate. Thus if $\ell=1$ part 5 of the theorem is trivial.

Assume that $\ell>1$. Consider all minimal weak optimal $\tilde{\mathbf{y}}$ such that $\operatorname{supp} \tilde{\mathbf{y}}=\operatorname{supp} \mathbf{y}^{\prime}$. Let $\mathcal{K}(\tilde{\mathbf{y}})=$ $\left\{k \in[m],(A \tilde{\mathbf{y}})_{k}=\hat{\rho}(A, B)(B \tilde{\mathbf{y}})_{k}\right\}$. Choose a minimal weak optimal y such that $|\mathcal{K}(\mathbf{y})|=p$ is maximal. We claim that $p \geq \ell$. Suppose not. Let $\mathcal{K}=\mathcal{K}(\mathbf{y})$. Assume the notations of part 2. Let $\tilde{A}=A^{\prime}[\mathcal{K},[n]], \tilde{B}=$ $B^{\prime}[\mathcal{K},[n]], \tilde{C}(t)=C(t)[\mathcal{K},[n]]$. Hence $\left.\tilde{C}\left(t_{0}\right)\right) \mathbf{z}=\mathbf{0}$. Suppose first that rank $\tilde{C}\left(t_{0}\right) \leq \ell-2$. Hence there exists $\mathbf{u} \in \mathbb{R}^{\ell}$ satisfying $\tilde{C}\left(t_{0}\right) \mathbf{u}=0$ such that $\mathbf{u}$ has positive and negative coordinates. Thus $\mathbf{z}$ and $\mathbf{u}$ are linearly independent. Let $\mathbf{v} \in \mathbb{R}^{m}$ be the extension of $\mathbf{u}$ by adding zero coordinates. In particular, $\mathbf{v}$ has a zero coordinate where $\mathbf{y}$ has zero coordinate. Let $s \geq 0$ and consider $\mathbf{z}(s)=\mathbf{z}+s \mathbf{u}$ and $\mathbf{y}(s)=\mathbf{y}+s \mathbf{v}$. Let $s_{1}>0$ be the smallest value such that $\mathbf{z}\left(s_{1}\right) \geq \mathbf{0}$ and $z\left(s_{1}\right)$ has at least one zero coordinate. Since $\mathbf{y}$ was minimal we deduce that $\mathbf{y}\left(s_{1}\right)$ is not weak optimal. That is, there exists $s_{2} \in\left(0, s_{1}\right)$ with the following property: There exists $j \in[m] \backslash \mathcal{K}$ such that $\left(A \mathbf{y}\left(s_{2}\right)\right)_{j}=t_{0}\left(B \mathbf{y}\left(s_{2}\right)\right)_{j}$ and $\left(A \mathbf{y}\left(s_{2}\right)\right)_{k} \leq t_{0}\left(B \mathbf{y}\left(s_{2}\right)\right)_{k}$ for $k \in[m] \backslash\{\mathcal{K} \cup\{j\}\}$. Clearly $\left(A \mathbf{y}\left(s_{2}\right)\right)_{k}=t_{0}\left(B \mathbf{y}\left(s_{2}\right)\right)_{k}$ for $k \in \mathcal{K} \cup\{j\}$. So $\mathbf{y}\left(s_{2}\right)$ is optimal and $\left|\mathcal{K}\left(\mathbf{y}\left(s_{2}\right)\right)\right|>|\mathcal{K}|=|\mathcal{K}(\mathbf{y})|$. This contradicts the choice of $\mathbf{y}$.

It is left to consider the case where $|\mathcal{K}|=\ell-1$ and rank $\tilde{C}\left(t_{0}\right)=\ell-1$. We proceed similarly as in the proof of (2). Permute the columns of $C(t)$ such that $\tilde{C}(t)=\left[\tilde{C}_{1}(t) \tilde{C}_{2}(t)\right] \in \mathbb{R}^{(\ell-1) \times \ell}$ and $\tilde{C}_{1}\left(t_{0}\right) \in \mathbb{R}^{(\ell-1) \times(\ell-1)}$ is a nonsingular matrix. Therefore $\tilde{C}_{1}(t)$ is nonsingular for $\left|t-t_{0}\right|<\varepsilon$ for some $\varepsilon>0$. Assume that $\mathbf{z}^{\top}=\left(\mathbf{u}^{\top}, \mathbf{v}^{\top}\right)$. Thus $\mathbf{u}=-\left(\tilde{C}\left(t_{0}\right)\right)^{-1} \tilde{C}_{1}\left(t_{0}\right) \mathbf{v}$. As $\mathbf{u}>0$ it follows that $\mathbf{u}(t)=-(\tilde{C}(t))^{-1} \tilde{C}_{1}(t) \mathbf{v}>0$ for $\left|t-t_{0}\right|<\varepsilon^{\prime}$ for some $\varepsilon^{\prime} \in(0, \varepsilon)$. Let $\mathbf{w}(t)^{\top}=\left(\mathbf{u}(t)^{\top}, \mathbf{v}^{\top}\right)$. As $\left(A^{\prime} \mathbf{w}\left(t_{0}\right)\right)_{j}<\left(t_{0}-\varepsilon_{1}\right)\left(B^{\prime} \mathbf{w}\left(t_{0}\right)\right)_{j}$ for $j \in[m] \backslash \mathcal{K}$ and some $\varepsilon_{1}>0$, there exists $t^{\prime} \in\left(t_{0}-\varepsilon^{\prime}, t_{0}\right)$ such that $\tilde{C}\left(t^{\prime}\right) \mathbf{w}(t)=\mathbf{0}$ and $\left(A^{\prime} \mathbf{w}\left(t^{\prime}\right)\right)_{j}<t^{\prime}\left(B^{\prime} \mathbf{w}\left(t^{\prime}\right)\right)_{j}$ for 
$j \in[m] \backslash \mathcal{K}$. That is, $\hat{\rho}\left(A^{\prime}, B^{\prime}\right) \leq t^{\prime}$. This contradicts our assumption that $\hat{\rho}\left(A^{\prime}, B^{\prime}\right)=t_{0}$. Therefore $|\mathcal{K}| \geq \ell$.

Parts (4)-(5) and their proof yield:

Corollary 5.2. Let $m, n$ be positive integers. Assume that $A, B \in \mathbb{R}_{+}^{m \times n}$. Suppose that $\hat{\rho}(A, B)<\infty$. Then there exists a minimal weak optimal $\mathbf{y} \in \mathbb{R}_{+}^{n}$ with at most $m$ positive coordinates, and a minimal $\mathcal{I} \subset[m]$, possibly an empty set, with the following properties:

$$
A(\mathcal{I}, \emptyset) \mathbf{y}=\hat{\rho}(A, B) B(\mathcal{I}, \emptyset) \mathbf{y}, \quad m-|\mathcal{I}| \geq|\operatorname{supp} \mathbf{y}| .
$$

Furthermore if $m-|\mathcal{I}|=|\operatorname{supp} \mathbf{y}|$ then the only nonzero solution of $(A(\mathcal{I}), \emptyset)-\hat{\rho}(A, B) B(\mathcal{I}, \emptyset)) \mathbf{x}=\mathbf{0}$ whose nonzero coordinates lie in supp $\mathbf{y}$ are multiples of $\mathbf{y}$.

We now show that some similar results apply to optimal vectors.

Theorem 5.3. Let $m, n$ be positive integers. Assume that $A=\left[a_{i j}\right], B=\left[b_{i j}\right] \in \mathbb{R}_{+}^{m \times n}$. Suppose that $\rho(A, B)<\infty$.

(1) Assume that $\mathbf{y} \in \mathbb{R}_{+}^{n} \backslash\{\mathbf{0}\}$ is optimal. Then at least one coordinate of $(A-\rho(A, B) B) \mathbf{y}$ is zero.

(2) Assume that there exists an optimal vector $\mathbf{y} \in \mathbb{R}_{+}^{n}$ with $\ell$ positive coordinates. Let $A^{\prime}, B^{\prime} \in \mathbb{R}^{m \times \ell}$ be the submatrices of $A$ and $B$ respectively induced by the positive coordinates of $\mathbf{y}$. If $\ell \geq m$ then $\operatorname{rank}\left(A^{\prime}-\rho(A, B) B^{\prime}\right)<m$.

(3) There exists an optimal $\mathbf{y}$ has at most $m$ positive coordinates.

Proof. (1) Part 2 of Lemma 2.6 yields the existence of a sequence $\mathbf{y}_{l} \in \Pi_{n}^{o}$ such that $\lim _{l \rightarrow \infty} \mathbf{y}_{l}=\mathbf{y}$, and $\lim _{l \rightarrow \infty} r\left(A, B, \mathbf{y}_{l}\right)=r(A, B, \mathbf{y})=\rho(A, B)$. Clearly, at least one coordinates of $\left(A-r\left(A, B, \mathbf{y}_{l}\right) B\right) \mathbf{y}_{l}$ is zero. Hence there exists an infinite subsequence $\left\{l_{p}\right\}, p \in \mathbb{N}$ such that a fixed coordinate of $\left(A-r\left(A, B, \mathbf{y}_{l}\right) B\right) \mathbf{y}_{l_{p}}$ is zero. Letting $p \rightarrow \infty$ we deduce the claim.

(2) Let $t_{0}=\rho(A, B)$. Assume first that $B \mathbf{y}>\mathbf{0}$. Then part (3) of Lemma2.5yields that $r(A, B, \mathbf{x}) \geq \rho(A, B)$ for each $\mathbf{x} \in \mathbb{R}_{+}^{n}, \sup \mathbf{x}=\sup \mathbf{y}$. Then we proceed as in the proof of part 2 of Theorem [5.1] using the notations and the results in this proof. Rename the columns of $A$ and $B$ so that the first $\ell$ coordinates of $\mathbf{y}$ are positive. Let $A^{\prime}=A[[m],[\ell]], B^{\prime}=B[[m],[\ell]], C(t)=A^{\prime}-t B^{\prime}$, and $\mathbf{0}<\mathbf{z} \in \mathbb{R}^{\ell}$ the projection of $\mathbf{y}$ on its first $\ell$ coordinates. As $B \mathbf{y}=B^{\prime} \mathbf{z}>\mathbf{0}$ it follows that we can choose $t_{1}<t_{0}$ such that $B^{\prime} \mathbf{z}^{\prime}>\mathbf{0}$. Hence $r\left(A^{\prime}, B^{\prime}, \mathbf{z}^{\prime}\right) \leq t_{1}<t_{0}$ which contradicts the claim that $r\left(A^{\prime}, B^{\prime}, \mathbf{z}^{\prime}\right) \geq t_{0}$. Hence rank $C\left(t_{0}\right)<m$.

Assume that $\mathcal{I} \neq \emptyset$ is the set of zero coordinates of $B \mathbf{y}$. That is $\mathcal{I}$ is the set of the zero rows of $B^{\prime}$. As $\rho(A, B)<\infty$ we deduce that the set of zero rows of $A^{\prime}$ contains $\mathcal{I}$. Clearly, rank $C\left(t_{0}\right)<m$.

(3) We prove the claim by induction on $n$. For $n=1$ the vector $\mathbf{y}=(1)$ is optimal, hence $m \geq \ell=1$. Assume that the claim holds for $n \leq N$ and suppose that $n=N+1$. Let $\mathbf{y}$ be a minimal optimal with vector with the minimal number of positive coordinates $\ell$. Assume the conventions of parts (1)-(2) of the proof. Suppose to the contrary that $\ell>m$. Then we proceed to the proof of part (3) of Theorem 5.1. Let $t_{0}$ and $\mathbf{0}<\mathbf{z} \in \mathbb{R}^{\ell}$ be as in part 2. Let $\mathcal{J}=\{\ell+1, \ldots, n\}$. (If $\ell=n$ then $\mathcal{J}=\emptyset$.) Assume that $\mathbf{x} \in \operatorname{ker} C\left(t_{0}\right) \backslash\{\mathbf{0}\}$ such that $\mathbf{x}$ has at least one negative coordinate and one positive coordinate. Set $\mathbf{z}(s)=\mathbf{z}+s \mathbf{x}$ and choose $s_{0}>0$ the biggest $s$ such that $\mathbf{z}\left(s_{0}\right) \geq \mathbf{0}$. Then $\mathbf{z}^{\prime}=\mathbf{z}\left(s_{0}\right) \geqslant \mathbf{0}$ and $\mathbf{z}^{\prime}$ has at least one zero coordinate. Clearly, $r\left(A^{\prime}, B^{\prime}, \mathbf{z}^{\prime}\right) \leq r\left(A^{\prime}, B^{\prime}, \mathbf{z}\right)=t_{0}$.

Suppose first that $r\left(A^{\prime}, B^{\prime}, \mathbf{z}^{\prime}\right)<t_{0}$. Part (2) of Lemma 2.5 yields that $B^{\prime} \mathbf{z}^{\prime}$ is not a positive vector. Hence $B^{\prime}$ has at least one zero row. Let $\mathcal{I}$ be the set of zero rows of $B$. As $r\left(A^{\prime}, B^{\prime}, \mathbf{z}^{\prime}\right)<t_{0}$ it follows that the set of zero rows of $A$ contains $\mathcal{I}$. Hence $\hat{\rho}\left(A^{\prime}, B^{\prime}\right) \leq r\left(A^{\prime}, B^{\prime}, \mathbf{z}^{\prime}\right)<t_{0}$.

Assume first that $|\mathcal{I}|=m$. Then $A^{\prime}=B^{\prime}=0$. If $\mathcal{J}=\emptyset$ then $A=A^{\prime}, B=B^{\prime}$, and a vector $(1,0, \ldots, 0)^{\top}$ is a minimal optimal vector, contrary to our assumption. Hence $|\mathcal{J}| \geq 1$. Clearly, $\rho(A, B)=\rho(A[[m],[\mathcal{J}]], B[[m],[\mathcal{J}]])$ and an optimal vector of $(A[[m],[\mathcal{J}]], B[[m],[\mathcal{J}]])$ can be extended trivially, by adding zero coordinates, to an optimal vector of $(A, B)$. The induction hypothesis on the pair $(A[[m],[\mathcal{J}]], B[[m],[\mathcal{J}]])$ yields the existence of an optimal $\mathbf{w} \in \mathbb{R}_{+}^{n-\ell}$ satisfying $|\sup \mathbf{w}| \leq m$. Extend trivially $\mathbf{w}$ to $\mathbb{R}_{+}^{n}$ to obtain an optimal vector $\mathbf{y}$ with $\sup \mathbf{y} \leq m$, contrary to our assumption.

Assume that $|\mathcal{I}|=m-m^{\prime} \in[m-1]$. Rename the rows of $A$ and $B$ such that $\mathcal{I}=\left\{m^{\prime}+1, \ldots, m\right\}$. Let $C=A\left[\left[m^{\prime}\right],[\ell]\right], D=B\left[\left[m^{\prime}\right],[\ell]\right]$. Clearly $\hat{\rho}(C, D)=\hat{\rho}\left(A^{\prime}, B^{\prime}\right)<t_{0}$. Let us consider a weak minimal vector $\mathbf{w} \in \mathbb{R}_{+}^{\ell}$ corresponding to $(C, D)$, with the minimal number of positive coordinates. Theorem 5.1 yields that $p=|\sup \mathbf{w}| \leq m^{\prime}$. Rename the first $\ell$ columns of $A$ and $B$ such that the first $p$ coordinates of $\mathbf{w}$ are positive. Let $\mathbf{0}<\mathbf{u} \in \mathbb{R}^{p}$ be the projection of $\mathbf{w}$ on its first $p$-coordinates. Let $C^{\prime}=C\left[\left[m^{\prime}\right],[p]\right], D^{\prime}=D\left[\left[m^{\prime}\right],[p]\right]$ 
Observe that $\hat{\rho}(C, D)=r(C, D, \mathbf{w})=r\left(C^{\prime}, D^{\prime}, \mathbf{u}\right)=\hat{\rho}\left(C^{\prime}, D^{\prime}\right)<t_{0}$. Let $\mathcal{I}^{\prime}$ be the set of zero rows of $D^{\prime}$. We claim that $m^{\prime} \geq p+\left|\mathcal{I}^{\prime}\right|$. Recall the set of zero rows in $C^{\prime}$ contains $\mathcal{I}^{\prime}$. Let $A_{11}=C^{\prime}\left(\mathcal{I}^{\prime}, \emptyset\right), B_{11}=D^{\prime}\left(\mathcal{I}^{\prime}, \emptyset\right)$. Clearly, $\hat{\rho}\left(C^{\prime}, D^{\prime}\right)=\hat{\rho}\left(A_{11}, B_{11}\right)$. Furthermore, $\mathbf{x} \in \mathbb{R}_{+}^{p}$ is a weak optimal vector of $\left(A_{11}, B_{11}\right)$ if and only if it is a weak optimal vector of $\left(C^{\prime}, D^{\prime}\right)$. Note that each weak optimal vector of $\left(C^{\prime}, D^{\prime}\right)$ can be trivially extended to a weak optimal vector of $(C, D)$. Since a weak optimal vector of $(C, D)$ has at least $p$ positive coordinates, it follows that each optimal vector of $\left(A_{11}, B_{11}\right)$ is positive. Hence $\rho\left(A_{11}, B_{11}\right)=\hat{\rho}\left(A_{11}, B_{11}\right)<t_{0}$.

Apply Theorem 5.1 to minimal optimal vectors of $\left(A_{11}, B_{11}\right)$ to deduce the inequality $m^{\prime}-\left|\mathcal{I}^{\prime}\right| \geq p$. Let $m^{\prime \prime}=m^{\prime}-\left|\mathcal{I}^{\prime}\right|$ rearrange the first $m^{\prime}$ rows of $A$ and $B$ such that $\mathcal{I}=\left\{m^{\prime \prime}+1, \ldots, m^{\prime}\right\}$. Let $\mathcal{K}=\{p+1, \ldots, n\}$. Observe

$$
\begin{aligned}
& A=\left[\begin{array}{ll}
A_{1} & A_{2}
\end{array}\right], A_{1}=A[[m],[p]]=\left[\begin{array}{c}
A_{11} \\
0
\end{array}\right], A_{2}=A[[m],[\mathcal{K}]]=\left[\begin{array}{c}
A_{12} \\
A_{22}
\end{array}\right], \\
& B=\left[\begin{array}{ll}
B_{1} & B_{2}
\end{array}\right], B_{1}=B[[m],[p]]=\left[\begin{array}{c}
B_{11} \\
0
\end{array}\right], B_{2}=B[[m],[\mathcal{K}]]=\left[\begin{array}{c}
B_{12} \\
B_{22}
\end{array}\right], \\
& A_{11}, B_{11} \in \mathbb{R}_{+}^{m^{\prime \prime} \times p}, \quad A_{22}, B_{22} \in \mathbb{R}^{\left(m-m^{\prime \prime}\right) \times(n-p)} .
\end{aligned}
$$

As $\mathcal{I}^{\prime}$ was the set of zero rows of $D^{\prime}$ we deduce that $B_{11}$ has no zero rows. Hence $B_{11} \mathbf{1}_{p}>\mathbf{0}$. Thus the conditions of part (3) of Lemma 2.5 hold. Thus $\rho\left(A_{22}, B_{22}\right)=t_{0}$. We now can apply the induction hypothesis on the optimal vector of the pair $\left(A_{22}, B_{22}\right)$. That is, there exists an optimal $\mathbf{v} \in \mathbb{R}_{+}^{n-p}$ satisfying $|\sup \mathbf{u}| \leq m-m^{\prime \prime}$. We now use the arguments of the proof of part (2) of Lemma 2.6 to deduce that $\mathbf{y}=\left(\mathbf{u}^{\top}, s_{1} \mathbf{v}^{\top}\right)^{\top}$ is an optimal for the pair $(A, B)$ for some $s_{1}>0$. Note that $|\sup \mathbf{y}|=|\sup \mathbf{u}|+|\sup \mathbf{v}| \leq$ $p+m-m^{\prime \prime} \leq m^{\prime \prime}+\left(m-m^{\prime \prime}\right)=m$, which contradicts our assumption.

It is left to discuss the case where $r\left(A^{\prime}, B^{\prime}, \mathbf{z}\left(s_{0}\right)\right)=\rho(A, B)$. Let $\hat{\mathbf{x}} \in \mathbb{R}^{n}$ be the trivial extensions of $\mathbf{x}$ to $\mathbb{R}^{n}$. Then $A \hat{\mathbf{x}}=t_{0} \hat{\mathbf{x}}$. Let $\mathbf{y}(s)=\mathbf{y}+s \hat{\mathbf{x}} \in \mathbb{R}^{n}$. Then $\left(A-t_{0} B\right) \mathbf{y}(s)=\left(A-t_{0} B\right) \mathbf{y} \leq \mathbf{0}$. Hence $r(A, B, \mathbf{y}(s)) \leq t_{0}$ for $\mathbf{y}(s) \geq \mathbf{0}$. This holds for $s \in\left[0, s_{0}\right]$. Note that our assumption is that $r\left(A, B, \mathbf{y}\left(s_{0}\right)\right)=t_{0}$. We claim that $\mathbf{y}\left(s_{0}\right)$ is optimal. Recall that there exists a sequence $\mathbf{0}<\mathbf{y}_{k} \in \mathbb{R}^{n}$ such that $\lim _{k \rightarrow \infty} \mathbf{y}_{k}=\mathbf{y}, \lim _{k \rightarrow \infty} r\left(A, B, \mathbf{y}_{k}\right)=r(A, B, \mathbf{y})=t_{0}>0$. Let $\mathbf{y}_{l}(s)=\mathbf{y}_{l}+s \hat{\mathbf{x}}$. Then

$$
\left(A-t_{0} B\right) \mathbf{y}_{k}(s)=\left(A-t_{0} B\right) \mathbf{y}_{k} \leq\left(r\left(A, B, \mathbf{y}_{k}\right)-t_{0}\right) \mathbf{y}_{k}, \quad k \in \mathbb{N} .
$$

Let $\mathbf{y}_{k}=\tilde{\mathbf{y}}_{k}+\mathbf{v}_{k}$, where $\tilde{\mathbf{y}}_{k}$ is obtained by replacing the positive coordinates of $\mathbf{y}_{k}$ with zero coordinates in the places $\mathbf{y}$ has zero coordinates. Then $\mathbf{v}_{k}=\mathbf{y}_{k}-\tilde{\mathbf{y}}_{k} \geq \mathbf{0}$. The coordinates of $\mathbf{v}_{k}$ are positive where the coordinates of $\tilde{\mathbf{y}}_{k}$ are zero, and the coordinates of $\mathbf{v}_{k}$ are zero where the coordinates of $\tilde{\mathbf{y}}_{k}$ are positive. Thus $\mathbf{y}_{k}(s)=\mathbf{y}(s)+\left(\tilde{\mathbf{y}}_{k}-\mathbf{y}\right)+\mathbf{v}_{k}$. Fix $s \in\left(0, s_{0}\right)$. We claim that there exists $K(s)>1$ and $N(s)>0$ such that for $k>N(s)$ we have the inequality $\mathbf{y}_{k} \leq K(s) \mathbf{y}_{k}(s)$. Indeed as $\mathbf{y}(s)$ has positive coordinates where $\mathbf{y}$ has positive coordinates it follows that there exists $K(s)>1$ such that $\mathbf{y} \leq \frac{K(s)}{2} \mathbf{y}(s)$. Clearly $\mathbf{v}_{k} \leq K(s) \mathbf{v}_{k}$ for all $k \in \mathbb{N}$. As $\lim _{k \rightarrow \infty}\left(\tilde{\mathbf{y}}_{k}-\mathbf{y}\right)=\mathbf{0}$ it follows that there exists $N(s)$ such that $\left(\tilde{\mathbf{y}}_{k}-\mathbf{y}\right) \leq \frac{K(s)}{2} \mathbf{y}(s)$ for $k>N(s)$. Hence $\mathbf{y}_{k} \leq K(s) \mathbf{y}_{k}(s)$ and $\mathbf{y}_{k}(s)>\mathbf{0}$ for $k>N(s)$. Therefore $r\left(A, B, \mathbf{y}_{k}(s)\right) \geq t_{0}$ for $k>N(s)$. Use (18) to deduce that $r\left(A, B, \mathbf{y}_{k}(s)\right) \leq r\left(A, B, \mathbf{y}_{k}\right)$ for $k>N(s)$.

Choose a sequence an increasing sequence $0<s_{1}<s_{2}<\cdots$ which converges to $s_{0}$. Choose an increasing subsequence $l_{j}, j \in \mathbb{N}$ such that $l_{j}>N\left(s_{j}\right),\left|\mathbf{y}_{l_{j}}\left(s_{j}\right)-\mathbf{y}\left(s_{j}\right)\right|<\frac{1}{j}$. and $r\left(A, B, \mathbf{y}_{j}\left(s_{j}\right)\right) \in\left[t_{0}, t_{0}+\frac{1}{j}\right]$. So $\lim _{j \rightarrow \infty} \mathbf{y}_{l j}\left(s_{j}\right)=\mathbf{y}\left(s_{0}\right)$ and $\mathbf{y}\left(s_{0}\right)$ is optimal. This contradicts our assumption that $\mathbf{y}$ is optimal vector with the minimum number of positive coordinates.

We now give a simple example of two positive invertible stochastic matrices $A, B \in \mathbb{R}_{+}^{2 \times 2}$ for which there is a unique optimal $\mathbf{y} \in \Pi_{2}$ with one positive coordinate.

Proposition 5.4. Let

$$
A=\left[\begin{array}{cc}
a & 1-a \\
b & 1-b
\end{array}\right], B=\left[\begin{array}{cc}
1-a & a \\
1-b & b
\end{array}\right], \quad 0<b<a<\frac{1}{2} .
$$

Then $\rho(A, B)=\hat{\rho}(A, B)=\frac{a}{1-a}<1$ and $\mathbf{z}=(1,0)^{\top}$ is the unique optimal vector in $\Pi_{2}$, which is not a $G P F$-eigenvector.

Proof. As $a, b \in\left(0, \frac{1}{2}\right)$ it follows that

$$
\min _{\mathbf{x} \in \Pi_{2}} \frac{(A \mathbf{x})_{1}}{(B \mathbf{x})_{1}}=\frac{(A \mathbf{z})_{1}}{(B \mathbf{z})_{1}}=\frac{a}{1-a}, \quad \min _{\mathbf{x} \in \Pi_{2}} \frac{(A \mathbf{x})_{2}}{(B \mathbf{x})_{2}}=\frac{(A \mathbf{z})_{2}}{(B \mathbf{z})_{2}}=\frac{b}{1-b},
$$


where $\mathbf{z}=(1,0)^{\top} \in \Pi_{2}$ is the unique vector that minimizes both ratios. As $\frac{b}{1-b}<\frac{a}{1-a}$ we deduce that $\rho(A, B)=\frac{a}{1-a}$ and $\mathbf{z}$ is a unique optimal in $\Pi_{2}$. Clearly, $\rho(A, B)<1$.

Note that rank $(A-\rho(A, B) B)=2$, which does not contradict part 2 of Theorem 5.1 as $\ell=1<m=2$. Observe that $A \mathbf{1}_{2}=B \mathbf{1}_{2}$, that is 1 is the eigenvalue of the generalized eigenvalue problem (3) with a corresponding positive eigenvector $\mathbf{1}_{2}$. Note that the second eigenvalue of (3) is $\lambda=-1$ with a corresponding eigenvector $(1,-1)^{\top}$.

Recall that for $A \in \mathbb{R}_{+}^{n \times n}$ we have that $\rho\left(A^{\top}\right)=\rho(A)$. For a pair of $A, B \in \mathbb{R}_{+}^{m \times n}$ such equality does not always hold. For a pair of matrices given in Proposition 5.4 we have $\rho\left(A^{\top}, B^{\top}\right)=\frac{1-a}{a}>\rho(A, B)$. Indeed, observe that $\frac{\left(A^{\top} \mathbf{x}\right)_{1}}{(B \mathbf{x})_{1}}<\frac{\left(A^{\top} \mathbf{x}\right)_{2}}{(B \mathbf{x})_{2}}$ for each $\mathbf{x} \in \Pi_{2}$. The minimum of the bigger ratio is achieved for $\mathbf{z}=(1,0)^{\top}$, which yields the equality $\rho\left(A^{\top}, B^{\top}\right)=\frac{1-a}{a}$.

\section{A SPECIAL $B$ APPEARING IN A WIRELESS NETWORK}

Definition 6.1. A pair $A, B \in \mathbb{R}_{+}^{m \times n}$ is called a WN-pair, (a wireless network pair), if $n \geq m, B$ has no zero row and each column of $B$ has exactly one positive entry.

A WN-pair was considered in [2]. It has the following interpretation in a wireless network [2, Introduction]. Each row $i$ in $A=\left[a_{i j}\right]$ corresponds to the entity $i$ (receiver), and each nonzero element in the row $i$ of $B=\left[b_{i j}\right]$ corresponds to an affector (transmitter) of the entity $i$. In the classical case, $m=n$ and $B$ is a diagonal matrix with positive diagonal. That is, each entity $i$ has one affector located at the entry $(i, i)$ of $B$. In more general case the entity $i$ may have several affectors corresponding to the positive entries in the row $i$ of $B$. The assumption that each column $B$ has one positive entry means that two different entities do not share a common affector. In view of the wireless network interpretation of the entries of $A$ and $B$, it is assumed in [2] that $a_{i j} b_{i j}=0$ for each pair $(i, j)$. In our treatment we drop this assumption.

Note that if $m=n$ then $B$ is called a monomial matrix. So $B=P D$, where $P$ is an $m \times m$ permutation matrix and $D$ is an $m \times m$ diagonal matrix with positive diagonal entires. Hence $B^{-1}=D^{-1} P^{\top}$.

The following theorem gives an explicit formula for $\hat{\rho}(A, B)$ of a WN-pair.

Theorem 6.2. Assume that $A, B \in \mathbb{R}_{+}^{m \times n}$ is a WN-pair. Let $\mathcal{E}(A, B) \subset \Pi_{n}$ be a finite set of vectors $\mathbf{w}$ that satisfy the following five conditions:

(1) The vector $\mathbf{w} \in \Pi_{n}$ has $\ell \leq m$ nonzero coordinates.

(2) Let $\mathcal{I}$ be the set of zero rows of $B[[m]$, supp $\mathbf{w}]$. Then $|\mathcal{I}|=m-\ell$. (Hence $B[[m] \backslash \mathcal{I}$, supp $\mathbf{w}]$ is monomial.)

(3) $A[\mathcal{I}$, supp $\mathbf{w}]=0$.

(4) The matrix $C(\mathbf{w})=B[[m] \backslash \mathcal{I} \text {, supp } \mathbf{w}]^{-1} A[[m] \backslash \mathcal{I}$, supp $\mathbf{w}]$ is irreducible.

(5) Let $\mathbf{0}<\mathbf{z} \in \mathbb{R}^{\ell}$ be the projection of $\mathbf{w}$ on supp $\mathbf{w}$. Then the vector $\mathbf{z}$ is the PF-eigenvector of $C(\mathbf{w})$. The above conditions imply that $\rho(C(\mathbf{w}))=r(A, B, \mathbf{w})$ and $A \mathbf{w}=\rho(C(\mathbf{w})) B \mathbf{w}$. Furthermore

$$
\hat{\rho}(A, B)=\min \{r(A, B, \mathbf{w}), \mathbf{w} \in \mathcal{E}(A, B)\}
$$

In particular, $\mathbf{y}$ is a minimal weak optimal if and only if $\mathbf{y} \in \mathcal{E}(A, B)$ and $\mathbf{y}$ minimizes the right hand side of (19). Furthermore, each minimal weak optimal is a weak GPF-eigenvector.

Proof. We first justify that the assumption $|\mathcal{I}|=m-l$ in part (2) implies that $B_{11}=B[[m] \backslash \mathcal{I}$, supp w is a monomial matrix. Since each column of $B$ has exactly one nonzero entries it follows that $B_{1}=B[[\mathrm{~m}]$, supp w] has $\ell$ nonzero entries. Since $|\mathcal{I}|=m-\ell$ it follows that $B_{11}$ has $\ell$ nonzero rows. That is, each row and column of $B_{11}$ has exactly one nonzero element. We next show that the conditions (1)-(5) imply that $A \mathbf{w}=\rho(C) B \mathbf{w}$. Let $A_{1}=A[[m]$, supp w $]$ and $A_{11}=A[[m] \backslash \mathcal{I}$, supp $w]$. Since $\mathbf{z}$ is a PF-eigenvector of $C(\mathbf{w})$ it follows that $A_{11} \mathbf{z}=\rho(C(\mathbf{w})) B_{11} \mathbf{z}$. Let $A_{21}=A\left[\mathcal{I}\right.$, supp w], $B_{21}=B\left[\mathcal{I}\right.$, supp w]. As $A_{21}=B_{21}=0$ we deduce that $A_{1} \mathbf{w}=\rho(C(\mathbf{w})) B_{1} \mathbf{w}$, which is equivalent to $A \mathbf{w}=\rho(C) B \mathbf{w}$. Hence $r(A, B, \mathbf{w})=\rho(C(\mathbf{w}))$.

In particular, $\hat{\rho}(A, B) \leq r(A, B, \mathbf{w})$. Denote by $\rho_{1}(A, B)$ the minimum in (19). Then $\hat{\rho}(A, B) \leq \rho_{1}(A, B)$. To show the equality (19) it is enough to show that a minimal weak optimal $\mathbf{y}$ is in $\mathcal{E}(A, B)$. We show this claim by induction on $m$.

For $m=1$ the equality (19) trivially holds. Assume that each minimal weak optimal $\mathbf{y}$ is in $\mathcal{E}(A, B)$ for $m \leq M$. Suppose that $m=M+1$. Assume that $\mathbf{y} \in \Pi_{n}$ is a minimal weak optimal vector with the $\ell=|\operatorname{supp} \mathbf{y}|$. Part 3 of Theorem 5.1 yields that $\ell \leq m$. Set $\mathbf{0}<\mathbf{z}$ be the projection of $\mathbf{y}$ on its support. 
Let $\mathcal{J}=[n] \backslash \operatorname{supp} \mathbf{y}, A^{\prime}=A(\emptyset, \mathcal{J})$ and $B^{\prime}=B(\emptyset, \mathcal{J})$. Then $\hat{\rho}(A, B)=\hat{\rho}\left(A^{\prime}, B^{\prime}\right)=r\left(A^{\prime}, B^{\prime}, \mathbf{z}\right)$. Denote by $\mathcal{I}$ the set of the zero rows of $B^{\prime}$. As $\hat{\rho}(A, B)<\infty$ it follows that $\mathcal{I}$ is a set of zero rows of $A^{\prime}$. Let $\tilde{A}=A(\mathcal{I}, \mathcal{J}), \tilde{B}=B(\mathcal{I}, \mathcal{J}) \in \mathbb{R}_{+}^{m^{\prime} \times l}$. Thus $\hat{\rho}(A, B)=\hat{\rho}(\tilde{A}, \tilde{B})=r(\tilde{A}, \tilde{B}, \mathbf{z})$. As $B$ does not have a zero column it follows that $B^{\prime}$ does not have a zero column. As $\mathcal{I}$ is the set of zero rows of $B^{\prime}$ it follows that $\tilde{B}$ does not have zero columns or zero rows. As each column of $\tilde{B}$ has one positive element it follows that $\tilde{B}$ has exactly $\ell$ nonzero entries. Hence $m^{\prime} \leq \ell$.

The equality $\hat{\rho}(A, B)=\hat{\rho}(\tilde{A}, \tilde{B})=r(\tilde{A}, \tilde{B}, \mathbf{z})$ yields that $\mathbf{z}$ is a weak optimal solution for $\hat{\rho}(\tilde{A}, \tilde{B})=\hat{\rho}(A, B)$. The assumption that $\mathbf{y}$ was minimal weak optimal yields that $\mathbf{z}$ a minimal weak optimal for $(\tilde{A}, \tilde{B})$.

Assume first that $m^{\prime}<m$. We apply the induction hypothesis to $\tilde{A}, \tilde{B}$ and a minimal $\mathbf{z}$ to deduce that $m^{\prime}=\ell$. So $\tilde{B}$ is a monomial matrix, $\hat{\rho}(\tilde{A}, \tilde{B})=\rho(C)$ and $\tilde{A} \mathbf{z}=\rho(C) \tilde{B} \mathbf{z}$. The induction hypothesis yields that $C$ is irreducible. Hence $\mathbf{y} \in \mathcal{E}(A, B)$ as we claimed.

It is left to discuss the case where $m^{\prime}=m=\ell$. In this case we use part 4 of Theorem 5.1, So $\mathbf{y}$ is a weak GPF-eigenvector. In particular $\tilde{A} \mathbf{z}=\hat{\rho}(A, B) \tilde{B} \mathbf{z}$. Since $m^{\prime}=m$ it follows that $\tilde{B}$ is a monomial matrix. Observe next that $\rho(C)=\hat{\rho}(\tilde{A}, \tilde{B})=\hat{\rho}(C, I)$. Furthermore, $\mathbf{z}>\mathbf{0}$ is a minimal weak optimal vector of $(C, I)$. Apply now part (4) of Theorem 3.2 A minimal weak optimal vector of $(C, I)$ is supported on $V_{i} \subseteq[m]$ which corresponds to a source in the reduced graph $\vec{G}_{r e d}$. Furthermore $A\left[V_{i}\right]$ is irreducible. Since $\mathbf{z}>\mathbf{0}$ it follows that $V_{i}=[m]$ and $C$ is irreducible. Hence $\mathbf{y} \in \mathcal{E}(A, B)$.

The following notion of $S$-irreducibility was introduced in [2]:

Definition 6.3. A WN-pair $A, B \in \mathbb{R}_{+}^{m \times n}$ is called $S$-irreducible if the following condition holds: For each subset $\mathcal{K} \subseteq[n]$ of cardinality $m$, such that $B[[m], \mathcal{K}]$ is a monomial matrix, the matrix $B[[m], \mathcal{K}]^{-1} A[[m], \mathcal{K}]$ is irreducible.

Note that if $n=m$ then $S$-irreducibility is equivalent to the irreducibility of $B^{-1} A$. The following proposition gives a sufficient condition for an $S$-irreducible pair:

Proposition 6.4. Let $A=\left[a_{i j}\right], B=\left[b_{i j}\right] \in \mathbb{R}_{+}^{m \times n}$ be a WN-pair. Assume that $a_{i j}>0$ if $b_{i j}=0$. Then the pair $A, B$ is irreducible.

Proof. Let $\mathcal{K} \subseteq[n]$ of cardinality $m$, such that $B_{1}=B[[m], \mathcal{K}]$ is a monomial matrix. Let $A_{1}=A[[m], \mathcal{K}]$. So $A_{1}$ has positive elements where the elements of $B_{1}$ are zero. Therefore all off-diagonal entries of $C=B_{1}^{-1} A_{1}$ are positive, and $C$ is irreducible.

The following theorem gives an explicit formula for $\rho(A, B)$ of WN-pair.

Theorem 6.5. Let $A, B \in \mathbb{R}_{+}^{m \times n}$ be a WN-pair. Denote by $\mathcal{M}(B)$ the subset of all $\mathcal{K} \subseteq[n]$ of cardinality $m$ such that the matrix $B[[m], \mathcal{K}]$ is monomial. Then

$$
\rho(A, B)=\min \left\{\rho\left(B[[m], \mathcal{K}]^{-1} A([m], \mathcal{K}]\right), \mathcal{K} \in \mathcal{M}(B)\right\}
$$

For each $\mathcal{K} \in \mathcal{M}(B)$ such that $\rho(A, B)=\rho\left(B[[m], \mathcal{K}]^{-1} A([m], \mathcal{K}]\right)$ there exists an optimal $\mathbf{y} \in \Pi_{n}$ with the following property: The support of $\mathbf{y}$ is contained in $\mathcal{K}$ and $\mathbf{y}$ is a GPF-vector. Assume that WN-pair is $S$-irreducible. Then each such $\mathbf{y}$ is minimal optimal.

Proof. Assume first that the pair $A, B$ is $S$-irreducible. Let $\mathcal{E}(A, B)$ be defined as in the Theorem 6.2. We claim that $|\operatorname{supp} \mathbf{w}|=m$ for each $\mathbf{w} \in \mathcal{E}(A, B)$. Assume to the contrary that $\ell=|\operatorname{supp} \mathbf{w}|<m$. After relabeling the elements of the set $[n]$ we can assume that supp $\mathbf{w}=[\ell]$. Let $\mathcal{I}$ be zero set of $B_{1}=B[[m],[\ell]]$. Recall that $B_{11}=B[[m] \backslash \mathcal{I},[\ell]]$ is monomial, and $A_{21}=A[\mathcal{I},[\ell]]=0$. Relabel the elements of $[m]$ such that $\mathcal{I}=\{\ell+1, \ldots, m\}$. Since $B$ does not have zero rows there is a subset $\mathcal{J}$ of $[n]$ of cardinality $m-\ell$ such that $B[\mathcal{I}, \mathcal{J}]$ is a monomial matrix. Clearly, $[\ell] \cap \mathcal{J}=\emptyset$. Let $\mathcal{K}=[\ell] \cup \mathcal{J}$. Then $B_{1}=B[[m], \mathcal{K}]$ is a monomial matrix, which is direct sum of $B_{11}$ and $B[\mathcal{I}, \mathcal{J}]$. Let $A_{1}=A[[m], \mathcal{K}]$. Since $A, B$ is $S$-ireducible it follows that $C=B_{1}^{-1} A_{1}$ is irreducible. This contradicts the fact that $C[\mathcal{I},[\ell]]=0$.

Thus for each $\mathbf{w} \in \mathcal{E}(A, B)$ we have that $|\operatorname{supp} \mathbf{w}|=m$. Let $\mathbf{z} \in \Pi_{m}^{o}$ be the projection of $\mathbf{w}$ on supp w. Then $C(\mathbf{w})=B[[m] \text {, supp } \mathbf{w}]^{-1} A[[m]$, supp $\mathbf{w}]$ is irreducible, and $\mathbf{z}$ is the PF-vector of $C(\mathbf{w})$. Hence $B \mathbf{w}=B[[m]$, supp $\mathbf{w}] \mathbf{z}>\mathbf{0}$. Theorem 6.2 yields that $\rho(C(\mathbf{w}))=r(A, B, \mathbf{w})$.

Vice versa, each $\mathcal{K} \in \mathcal{M}(B)$ induces $\mathbf{w} \in \mathcal{E}(A, B)$ as follows. Let $\mathbf{z} \in \Pi_{m}^{o}$ the PF-eigenvector of $C=$ $\left.B[[m], \mathcal{K}]^{-1} A([m], \mathcal{K}]\right)$. Then $\mathbf{w} \in \Pi_{n}$ is obtained from $\mathbf{z}$ by adding zero coordinates. As $C$ is irreducible we deduce that $\mathbf{w} \in \mathcal{E}(A, B)$. 
Recall that $\hat{\rho}(A, B)$ is given by (19). Assume that $\mathbf{y}$ is a minimal weak optimal. So $\mathbf{y} \in \mathcal{E}(A, B)$ and $B \mathbf{y}>\mathbf{0}$. Lemma 2.7 yields that $\mathbf{y}$ is minimal optimal. Hence $\rho(A, B)=\hat{\rho}(A, B)$. Characterization (20) follows from (19). Furthermore $\mathbf{y}$ is GPF-vector. This proves the theorem in the case where $A, B$ is $S$ irreducible.

Assume now that $A, B$ is not $S$-irreducible. Let $J \in \mathbb{R}^{m \times n}$ be a matrix whose all entries are 1 . For $l \in \mathbb{N}$ denote let $A_{l}=A+\frac{1}{l} J$. So $A_{l}>0$, and Proposition 6.4 implies that the pair $A_{l}, B$ is $S$-irreducible. Fix $\mathcal{K} \in \mathcal{M}(B)$. Let $\left.C=B[[m], \mathcal{K}]^{-1} A([m], \mathcal{K}]\right)$ and $\left.C_{l}=B[[m], \mathcal{K}]^{-1} A_{l}([m], \mathcal{K}]\right)$. Observe that $A_{l+1} \leq A_{l}$ for $l \in \mathbb{N}$. Hence $C_{l+1} \leq C_{l}$ for $l \in \mathbb{N}$, and $\lim _{l \rightarrow \infty} C_{l}=C$. Therefore $\rho\left(C_{l}\right), l \in \mathbb{N}$ is a decreasing sequence which converges to $\rho(C)$. Apply characterization (20) to $\rho\left(A_{l}, B\right)$. Let $\rho_{1}(A, B)$ be the right hand side of (20). It now follows that $\lim _{l \rightarrow \infty} \rho\left(A_{l}, B\right)=\rho_{1}(A, B)$. Part 5 of Lemma 2.7yields that $\rho(A, B)=\rho_{1}(A, B)$. Hence the characterization (20) holds.

Assume that $\mathcal{K} \in \mathcal{M}(B)$ and $\rho(A, B)=\rho\left(B[[m], \mathcal{K}]^{-1} A([m], \mathcal{K}]\right)$. For each $l \in \mathbb{N}$ let $\mathbf{w}_{l} \in \Pi_{n}$ be be the vector induced by the PF-eigenvector $\mathbf{0}<\mathbf{z}_{l} \in \Pi_{m}^{o}$ of $\left.B[[m], \mathcal{K}]^{-1} A_{l}([m], \mathcal{K}]\right)$. Let $r_{l}=\rho\left(B[[m], \mathcal{K}]^{-1} A_{l}([m], \mathcal{K}]\right)$. Then $A_{l} \mathbf{w}_{l}=r_{l} B \mathbf{w}_{l}$. Pick a convergent subsequence $\mathbf{w}_{l_{k}} \rightarrow \mathbf{y}, k \rightarrow \infty$. Then $A \mathbf{y}=\rho(A, B) B \mathbf{y}$.

We claim that $\mathbf{y}$ is optimal. Choose $\mathbf{x}_{l} \in \Pi_{n}^{o}$ such that $r\left(A_{l}, B, \mathbf{x}_{l}\right) \leq\left(\rho\left(A_{l}, B\right)+\frac{1}{l}\right) \mathbf{x}_{l}$. Clearly, $\rho(A, B) \leq$ $\rho\left(A_{l}, B\right) \leq r_{l}$. Set $\mathbf{v}_{l}=\left(1-\frac{1}{l}\right) \mathbf{y}+\frac{1}{l} \mathbf{x}_{l}>\mathbf{0}$. Then $\lim _{k \rightarrow \infty} \mathbf{v}_{l_{k}}=\mathbf{y}$, and $\lim _{k \rightarrow \infty} r\left(A_{l}, B, \mathbf{x}_{l}\right)=\rho(A, B)$. Hence $\mathbf{y}$ is optimal.

To summarize, if a WN-pair is $S$-irreducible then each minimal optimal y, which is a GPF-vector, and corresponds to an optimal choice of one transmitter for each receiver. If a WN-pair is not $S$-irreducible there exist an optimal $\mathbf{y}$, which is a GPF-vector, and corresponds to an optimal choice of one transmitter for each receiver. However, for some receivers all their transmitters may shut off. This can happen in the classical case where $m=n$ and $B=I_{m}$. For example:

$$
A=\left[\begin{array}{llll}
0 & 1 & 1 & 1 \\
1 & 0 & 1 & 1 \\
0 & 0 & 0 & 1 \\
0 & 0 & 1 & 0
\end{array}\right] .
$$

Then the only optimal $\mathbf{y} \in \Pi_{4}$ is $\mathbf{y}=\frac{1}{2}(1,1,0,0)^{\top}$.

\section{A PAIR OF CP-OPERATORS}

Recall that $\mathbb{C}^{n}$ is equipped with the standard inner product $\langle\mathbf{x}, \mathbf{y}\rangle=\mathbf{y}^{*} \mathbf{x}$. Given a finite dimensional inner product space over $\mathbb{C}$, with a product $\langle\cdot, \cdot\rangle$, we denote by $\mathrm{S}(\mathbf{V}) \supset \mathrm{S}_{+}(\mathbf{V}) \supset \mathrm{S}_{+, 1}(\mathbf{V})$ the real space of self adjoint operators $A: \mathbf{V} \rightarrow \mathbf{V}$, the cone of positive semidefinite operators and the convex set of all positive semidefinite operators with trace 1. By fixing an orthonormal basis $\mathbf{e}_{1}, \ldots, \mathbf{e}_{n}$ in $\mathbf{V}$ we identify $\mathrm{S}(\mathbf{V}), \mathrm{S}_{+}(\mathbf{V}), \mathrm{S}_{+, 1},(\mathbf{V})$ with $\mathrm{H}_{n}, \mathrm{H}_{+, n}, \mathrm{H}_{+, 1, n}$ respectively.

On $\mathbb{C}^{n \times n}$ we have the standard inner product $\langle U, V\rangle=\operatorname{tr} V^{*} U$. For $X \in \mathrm{H}_{n}$ we denote by $\lambda_{1}(A) \geq \cdots \geq$ $\lambda_{n}(X)$ the $n$-real eigenvalues of $X$ counted with their multiplicities. Recall that for $X, Y \in \mathrm{H}_{n}$ we say that $Y \succeq X$ if $Y-X \in \mathrm{H}_{+, n}$, i.e., $Y-X$ is positive semidefinite. Denote by $\mathrm{H}_{++, n}$ the interior of the cone $\mathrm{H}_{+, n}$, i.e., the open set of positive definite $n \times n$ Hermitian matrices. Then $Y \succ X$ if $Y-X \in \mathrm{H}_{++, n}$. Let $\mathrm{H}_{++, 1, n}=\mathrm{H}_{+, 1, n} \cap \mathrm{H}_{++, n}$. Denote by $C P(n, m)$ the cone of completely positive operators from $\mathrm{H}_{n}$ to $\mathrm{H}_{m}$, given by (9). In the rest of the paper we assume that $\mathcal{A}, \mathcal{B} \in C P(n, m)$. Then we can define $\rho(\mathcal{A}, \mathcal{B})$ and $\hat{\rho}(\mathcal{A}, \mathcal{B})$ as in (44), (15) and (6) with respect to the cones $\mathbf{K}_{1}=\mathrm{H}_{+, n}, \mathbf{K}_{2}=\mathrm{H}_{+, m}$. We call $Y \in \mathrm{H}_{+, n} \backslash\{0\}$ weak optimal if $\hat{\rho}(\mathcal{A}, \mathcal{B})=r(\mathcal{A}, \mathcal{B}, Y)$. We call $Y \in \mathrm{H}_{+, n} \backslash\{0\}$ optimal if the following conditions hold: First, $\rho(\mathcal{A}, \mathcal{B})=r(\mathcal{A}, \mathcal{B}, Y)$. Second, there exists a sequence $X_{l} \in \mathrm{H}_{++, n}$ such that $\lim _{l \rightarrow \infty} X_{l}=Y$ and $\lim _{l \rightarrow \infty} r\left(\mathcal{A}, \mathcal{B}, X_{l}\right)=r(\mathcal{A}, \mathcal{B}, Y)$. We say that $Y$ is a generalized Perron-Frobenius vector or weak generalized Perron-Frobenius vector if

$$
\mathcal{A}(Y)=\rho(A, B) \mathcal{B}(Y), \text { or } \mathcal{A}(Y)=\hat{\rho}(A, B) \mathcal{B}(Y), \quad Y \in \mathrm{H}_{+, n} \backslash\{0\},
$$

respectively.

Given a pair $\mathcal{C}, \mathcal{D} \in C P(n, m)$ we say that $\mathcal{C} \succeq \mathcal{D}$ or $\mathcal{C} \succ \mathcal{D}$ if for each $X \in \mathrm{H}_{+, 1, n}$ we have that $\mathcal{C}(X) \succeq \mathcal{D}(X)$ or $\mathcal{C}(X) \succ \mathcal{D}(X)$ respectively. If $\mathcal{D}=0$ the $\mathcal{C} \succ 0$ is called a positive CP-operator. An example of positive CP-operator $\mathcal{I}(n, m)$ is the operator $\mathcal{I}(n, m)(Z)=(\operatorname{tr} Z) I_{m}$ for any $Z \in \mathbb{C}^{n \times n}$. (We will justify briefly why $\mathcal{I}(n, m)$ is completely positive in the next section.) 
In this paper we will concentrate on $\hat{\rho}(\mathcal{A}, \mathcal{B})$ since this quantity is much easier to deal with. When the proofs of our results for CP-pair very similar for the matrix pair $A, B \in \mathbb{R}_{+}^{m \times n}$ we will omit the proofs.

For $\mathcal{A}, \mathcal{B} \in C P(n, m)$ and $X \in \mathrm{H}_{n,+, 1}$ we give a formula to compute $r(\mathcal{A}, \mathcal{B}, X)$. To do that we need to recall the classical definition of the Rayleigh quotient for $A, B \in \mathrm{H}_{+, m}$ [12, §4.4]:

Lemma 7.1. Let $A, B \in H_{+, m}$. Define

$$
\lambda(A, B)=\sup _{\mathbf{x} \in \mathbb{C}^{m} \backslash\{\mathbf{0}\}} \frac{\mathbf{x}^{*} A \mathbf{x}}{\mathbf{x}^{*} B \mathbf{x}} .
$$

Then the above supremum is achieved for some $\mathbf{y} \in \mathbb{C}^{m} \backslash\{\mathbf{0}\}$ :

(1) $\lambda(A, B)=\infty$ if and only if $\operatorname{ker} B$ is not a subset of $\operatorname{ker} A$. Then $\mathbf{y} \in \operatorname{ker} B \backslash \operatorname{ker} A$.

(2) If $A=B=0$ then $\lambda(A, B)=0$ and $\mathbf{y}$ is any nonzero vector in $\mathbb{C}^{m}$.

(3) Assume that $\operatorname{ker} B \subseteq \operatorname{ker} A$ and $\operatorname{dim} \operatorname{ker} B<m$. Let $\mathbf{V} \subseteq \mathbb{C}^{m}$ be the orthogonal complement of $\operatorname{ker} B$. Then $\mathbf{V}$ is an invariant subspace of $A$ and $B$. Denote by $A_{1}, B_{1}$ the restricitions of $A, B$ to $\mathbf{V}$. Then $B_{1}^{-1} A_{1}$ is a diagonalizable operator in $\mathbf{V}$, with nonnegative eigenvalues. Furthermore

$$
\lambda(A, B)=\lambda\left(A_{1}, B_{1}\right)=\rho\left(B_{1}^{-1} A_{1}\right) .
$$

Morevover, a maximizing $\mathbf{y}$ of the quotient (22) can be chosen to be an eigenvector of $B_{1}^{-1} A_{1}$ corresponding to $\rho\left(B_{1}^{-1} A_{1}\right)$.

In particular

$$
\lambda(A, B)=\inf \{t \geq 0, t B \succeq A\} .
$$

Proof. Parts (1) and (2) are straightforward. We now prove (3). Suppose first that ker $B=\{\mathbf{0}\}$. So $B \in \mathrm{H}_{++, m}$. Let $C=\sqrt{B}$ be the unique root of $B$ such that $C \in \mathrm{H}_{++, m}$. Set $\mathbf{x}=C^{-1} \mathbf{z}$. Then $\frac{\mathbf{x}^{*} A \mathbf{x}}{\mathbf{x}^{*} B \mathbf{x}}=\frac{\mathbf{z}^{*} C^{-1} A C^{-1} \mathbf{z}}{\mathbf{z}^{*} \mathbf{z}}$. Thus the supremum (22) is the maximum characterization of the maximum eigenvalue of $C^{-1} A C^{-1} \in \mathrm{H}_{+, m}$. So

$$
\lambda(A, B)=\lambda\left(C^{-1} A C^{-1}, I_{m}\right)=\rho\left(C^{-1} A C^{-1}\right)=\rho\left(C^{-2} A\right)=\rho\left(B^{-1} A\right) .
$$

As $C^{-1} A C^{-1} \in \mathrm{H}_{+, m}$ has nonnegative eigenvalues it follows that $B^{-1} A$ is diagonizable with nonnegative eigenvalues.

Suppose now that $1 \leq \operatorname{dim} \operatorname{ker} B<m$. Let $\mathbf{V}=\operatorname{ker} B^{\perp}$. Then $A \mathbf{V} \subseteq \mathbf{V}=B \mathbb{C}^{n}$. Suppose that $\mathbf{x} \in \operatorname{ker} B \backslash\{\mathbf{0}\}$. Then $\frac{\mathbf{x}^{*} A \mathbf{x}}{\mathbf{x}^{*} B \mathbf{x}}=\frac{0}{0}=0$. Assume that $\mathbf{x} \in \mathbb{C}^{m} \backslash \operatorname{ker} B$. Then $\mathbf{x}=\mathbf{u}+\mathbf{v}$, where $\mathbf{u} \in \mathbf{V} \backslash\{\mathbf{0}\}$ and $\mathbf{v} \in \operatorname{ker} B$. Clearly $\frac{\mathbf{x}^{*} A \mathbf{x}}{\mathbf{x}^{*} B \mathbf{x}}=\frac{\mathbf{u}^{*} A \mathbf{u}}{\mathbf{u}^{*} B \mathbf{u}}=\frac{\mathbf{u}^{*} A_{1} \mathbf{u}}{\mathbf{u}^{*} B_{1} \mathbf{u}}$. Apply the previous arguments to $A_{1}, B_{1} \in \mathrm{S}_{+}(\mathbf{V})$ to deduce that $\lambda(A, B)=\lambda\left(A_{1}, B_{1}\right)=\rho\left(B_{1}^{-1} A_{1}\right)$.

The characterization (24) follows straightforward from (22).

Corollary 7.2. Let $\mathcal{A}, \mathcal{B} \in C P(n, m)$ and $X \in H_{+, n} \backslash\{0\}$. Then $r(\mathcal{A}, \mathcal{B}, X)=\lambda(\mathcal{A}(X), \mathcal{B}(X))$.

Lemma 7.3. Let $\mathcal{A}, \mathcal{B} \in C P(n, m)$. Then

(1) $\hat{\rho}(\mathcal{A}, \mathcal{B}) \leq \rho(\mathcal{A}, \mathcal{B})$.

(2) Assume that $\mathcal{A}_{1}, \mathcal{B}_{1} \in C P(n, m)$ and $\mathcal{A}_{1} \preceq \mathcal{A}, \mathcal{B} \preceq \mathcal{B}_{1}$. Then $\rho\left(\mathcal{A}_{1}, \mathcal{B}_{1}\right) \leq \rho(\mathcal{A}, \mathcal{B})$ and $\hat{\rho}\left(\mathcal{A}_{1}, \mathcal{B}_{1}\right) \leq$ $\hat{\rho}(\mathcal{A}, \mathcal{B})$.

(3) There exists a weak optimal $Y \in H_{n,+, 1}$.

(4) Assume that there exists a weak optimal $Y$ such that either $\mathcal{B}(Y) \succ 0$ or $\mathcal{A}(Y) \succ 0$. If $\rho(\mathcal{A}, \mathcal{B})<\infty$ then $\rho(\mathcal{A}, \mathcal{B})=\hat{\rho}(\mathcal{A}, \mathcal{B})$.

(5) Suppose that $\rho(\mathcal{A}, \mathcal{B})<\infty$, and either $\mathcal{A} \succ 0$ or $\mathcal{B} \succ 0$. Then $\rho(\mathcal{A}, \mathcal{B})=\hat{\rho}(A, B)$.

(6) Assume that $0 \prec \mathcal{D}_{l} \in C P(n, m)$ for $l \in \mathbb{N}$ and $\lim _{l \rightarrow \infty} \mathcal{D}_{l}=0$. Then $\lim _{l \rightarrow \infty} \rho\left(\mathcal{A}, \mathcal{B}+\mathcal{D}_{l}\right)=\hat{\rho}(\mathcal{A}, \mathcal{B})$.

(7) Assume that $0 \prec \mathcal{D}_{l} \in C P(n, m)$ for $l \in \mathbb{N}$ and $\lim _{l \rightarrow \infty} \mathcal{D}_{l}=0$. If $\mathcal{B}\left(I_{n}\right) \succ 0$ then $\lim _{l \rightarrow \infty} \rho(\mathcal{A}+$ $\left.\mathcal{D}_{l}, \mathcal{B}\right)=\rho(\mathcal{A}, \mathcal{B})$.

Proof. (1) Trivial.

(2) Straightforward from the definitions.

(3). As in the proof of part (1) of Lemma 2.6

(4) As in the proof of part (2) of Lemma 2.7

(5) As in the proof of part (3) of Lemma 2.7 
(6) We use similar arguments to the proof of part (4) of Lemma 2.7 with the following modifications. Let $\mathcal{B}_{l}=\mathcal{C}_{l}+\mathcal{D}_{l}$, where $\mathcal{C}_{l}=\mathcal{B}$. Then the arguments of the part (4) of Lemma 2.7 apply.

(7) We use similar arguments to the proof of part (5) of Lemma 2.7 with the following modifications. Let $\mathcal{A}_{l}=\mathcal{C}_{l}+\mathcal{D}_{l}$, where $\mathcal{C}_{l}=\mathcal{A}$. Then the arguments of the part (5) of Lemma 2.7 apply.

Let $\mathcal{C}: \mathrm{H}_{n} \rightarrow H_{m}$ be a linear operator. Then there exists a dual operator $\mathcal{C}^{\vee}: \mathrm{H}_{m} \rightarrow \mathrm{H}_{n}$ which is defined as follows. Recall that on $\mathrm{H}_{n}$ one has the inner product $\langle X, Z\rangle=\operatorname{tr} X Z$, where $\operatorname{tr} W$ is the trace of the matrix $W \in \mathbb{C}^{n \times n}$. Then $\mathcal{C}^{\vee}: \mathrm{H}_{m} \rightarrow \mathrm{H}_{n}$ is defined uniquely by the property $\langle\mathcal{C}(X), Z\rangle=\left\langle X, \mathcal{C}^{\vee}(Z)\right\rangle$ for all $X \in \mathrm{H}_{n}$ and $Z \in \mathrm{H}_{m}$. Assume that $\mathcal{C}$ is CP-operator given by (9). Then $\mathcal{C}$ is called unital if $\mathcal{C}\left(I_{n}\right)=I_{m}$. Recall that $\mathcal{C}^{\vee}$ is also completely positive and given by $C^{\vee}(Y)=\sum_{j=1}^{k} T_{j}^{*} Y T_{j}$. Thus $\mathcal{C}$ is a quantum channel if and only if $\mathcal{C}^{\vee}$ is unital.

The following Lemma is an analog of Lemma 2.2

Lemma 7.4. Let $\mathcal{A}, \mathcal{B} \in C P(n, m)$. Then

(1) $\hat{\rho}(\mathcal{A}, \mathcal{B})=0$ if and only if $\mathcal{A}^{\vee}\left(I_{m}\right)$ is not positive definite.

(2) Assume that $\mathcal{A}^{\vee}\left(I_{m}\right)$ is positive definite. Then

$$
\hat{\rho}(\mathcal{A}, \mathcal{B}) \geq \rho\left(\mathcal{A}^{\vee}\left(I_{m}\right)^{-1} \mathcal{B}^{\vee}\left(I_{m}\right)\right)^{-1} .
$$

In particular, if $\mathcal{A}$ and $\mathcal{B}$ are quantum channels then $\rho(\mathcal{A}, \mathcal{B}) \geq 1$.

Proof. (1) Clearly, $\hat{\rho}(\mathcal{A}, \mathcal{B})=0$ if and only if there exists $X \in \mathrm{H}_{+, 1, n}$ such that $\mathcal{A}(X)=0$. Recall that $Y \in \mathrm{H}_{m,+}$ is zero if and only if $\left\langle Y, I_{m}\right\rangle=0$. So $\mathcal{A}(X)=0$ if and only if $\left\langle\mathcal{A}(X), I_{m}\right\rangle=\left\langle X, \mathcal{A}^{\vee}\left(I_{m}\right)\right\rangle=0$. Hence $\mathcal{A}^{\vee}\left(I_{m}\right) \nsucc 0$.

Vice versa, assume that $\mathcal{A}^{\vee}\left(I_{m}\right) \nsucc 0$. Therefore there exists a vector $\mathbf{x} \in \mathbb{C}^{n}, \mathbf{x}^{*} \mathbf{x}=1$, such that $\mathcal{A}^{\vee}\left(I_{m}\right) \mathbf{x}=\mathbf{0}$. In particular,

$$
0=\mathrm{x}^{*} \mathcal{A}^{\vee}\left(I_{m}\right) \mathrm{x}=\left\langle\mathrm{xx}^{*}, \mathcal{A}^{\vee}\left(I_{m}\right)\right\rangle=\left\langle\mathcal{A}\left(\mathrm{xx}^{*}\right), I_{m}\right\rangle \Rightarrow \mathcal{A}\left(\mathrm{xx}^{*}\right)=0 .
$$

(2) Assume that $\mathcal{A}^{\vee}\left(I_{m}\right) \succ 0$. Let $X \in \mathrm{H}_{n,+, 1}$. Assume that rank $X=r \in[n]$. Then spectral decomposiiton of $X$ is $X=\sum_{i=1}^{r} \lambda_{i} \mathbf{x}_{i} \mathbf{x}_{i}^{*}$, where each $\lambda_{i}>0$ and $\mathbf{x}_{j}^{*} \mathbf{x}_{i}=\delta_{i j}$ for $i, j \in[r]$. As $\mathcal{A}(X) \leq r(\mathcal{A}, \mathcal{B}, X) \mathcal{B}(X)$ it follows that

$$
\left\langle X, \mathcal{A}^{\vee}\left(I_{m}\right)\right\rangle=\left\langle\mathcal{A}(X), I_{m}\right\rangle \leq r(\mathcal{A}, \mathcal{B}, X)\left\langle\mathcal{B}(X), I_{m}\right\rangle=r(\mathcal{A}, \mathcal{B}, X)\left\langle X, \mathcal{B}^{\vee}\left(I_{m}\right)\right\rangle
$$

Hence

$$
\begin{aligned}
& r(\mathcal{A}, \mathcal{B}, X)^{-1} \leq \frac{\left\langle X, \mathcal{B}^{\vee}\left(I_{m}\right)\right\rangle}{\left\langle X, \mathcal{A}^{\vee}\left(I_{m}\right)\right\rangle}=\frac{\sum_{i=1}^{r} \lambda_{i} \mathbf{x}_{i}^{*} \mathcal{B}^{\vee}\left(I_{m}\right) \mathbf{x}_{i}}{\sum_{i=1}^{r} \lambda_{i} \mathbf{x}_{i}^{*} \mathcal{A}^{\vee}\left(I_{m}\right) \mathbf{x}_{i}} \\
& \leq \max _{i \in[r]} \frac{\mathbf{x}_{i}^{*} \mathcal{B}^{\vee}\left(I_{m}\right) \mathbf{x}_{i}}{\mathbf{x}_{i}^{*} \mathcal{A}^{\vee}\left(I_{m}\right) \mathbf{x}_{i}} \leq \lambda\left(\mathcal{B}^{\vee}\left(I_{m}\right), \mathcal{A}^{\vee}\left(I_{m}\right)\right)=\rho\left(\mathcal{A}^{\vee}\left(I_{m}\right)^{-1} \mathcal{B}^{\vee}\left(I_{m}\right)\right) .
\end{aligned}
$$

This establishes (25).

Assume that $\mathcal{A}$ and $\mathcal{B}$ are quantum channels. Then $\mathcal{A}^{\vee}\left(I_{m}\right)=\mathcal{B}^{\vee}\left(I_{m}\right)=I_{n}$. Hence $\rho\left(\mathcal{A}^{\vee}\left(I_{m}\right)^{-1} \mathcal{B}^{\vee}\left(I_{m}\right)\right)=$ 1 and then $\hat{\rho}(\mathcal{A}, \mathcal{B}) \geq 1$.

\section{Polynomial approximation of $\rho(\mathcal{A}, \mathcal{B})$ For $\delta$-Positive $\mathcal{B}$}

In this section we assume that $\mathcal{A}, \mathcal{B} \in C P(n, m)$. Suppose furthermore that $0<\rho(\mathcal{A}, \mathcal{B})<\infty$. We now want to apply the bisection algorithm as in the proof of Theorem 4.2. To this end, for a given $t>0$ we need to decide if the intersection $(t \mathcal{B}-\mathcal{A})\left(\mathrm{H}_{+, 1, n}\right) \cap \mathrm{H}_{+, m}$ is empty or not.

Let $X \in \mathrm{H}_{n}$ and $Z \in \mathrm{H}_{m}$. The square of the distance between $t \mathcal{B}(X)-\mathcal{A}(X)$ and $Z$ is given by the following quadratic convex function:

$$
f_{t}(X, Z)=\langle t \mathcal{B}(X)-\mathcal{A}(X)-Z, t \mathcal{B}(X)-\mathcal{A}(X)-Z\rangle, \quad X \in \mathrm{H}_{n}, Z \in \mathrm{H}_{m} .
$$

We assume that $(X, Z)$ are in the cone $\mathrm{H}_{+, n} \times \mathrm{H}_{+, m}$ subject to the linear constrain $\operatorname{tr} X=1$. Note that $f_{t}(X, Z) \geq 0$. Finding the distance between the two convex sets $(t \mathcal{B}-\mathcal{A})\left(\mathrm{H}_{+, 1, n}\right)$ and $\mathrm{H}_{+, m}$ is equivalent to the minimization problem

$$
\mu_{0}(t)=\min \left\{f_{t}(X, Z), X \in \mathrm{H}_{+, 1, n}, Z \in \mathrm{H}_{+, m}\right\} .
$$

This minimization problem can be dealt with by the standard interior point methods [5. Fix $\tau>0$. Assume that we found an approximation $\mu(t) \in \mathbb{Q}_{++}$of $\mu_{0}(t)$ by an interior method within precision $\tau$ in polynomial 
time in the data. If $\mu(t) \geq 2 \tau$ then $\operatorname{dist}\left((t \mathcal{B}-\mathcal{A})\left(\mathrm{H}_{+, 1, n}\right), \mathrm{H}_{+, m}\right) \geq \tau$. Hence $\rho(\mathcal{A}, \mathcal{B})>t$. Suppose that $\mu(t)<2 \tau$. How can we estimate from above $\rho(A, B)$ ? Recall that $\mu(t)=f_{t}(X(t), Z(t))$. So

$$
\begin{aligned}
& t \mathcal{B}(X(t))=\mathcal{A}(X(t))+Z(t)+W, \\
& X(t) \in \mathrm{H}_{++, 1, n}, Z(t) \in \mathrm{H}_{++, m}, W \in \mathrm{H}_{m},\|W\|^{2}=\operatorname{tr} W^{2}=\mu(t)<2 \tau .
\end{aligned}
$$

To find an upper bound for $\rho(\mathcal{A}, \mathcal{B})$ from (28) we need to assume a positivity condition on $\mathcal{B}$.

Definition 8.1. Assume that $m, n$ are two positive integers and $\delta \geq 0$. Then

(1) Denote by $\mathcal{I}(n, m): \mathrm{H}_{n} \rightarrow \mathrm{H}_{m}$ the linear transformation $\mathcal{I}(n, m)(X)=(\operatorname{tr} X) I_{m}$ for $X \in \mathrm{H}_{n}$.

(2) A real linear transformation $\mathcal{L}: \mathrm{H}_{n} \rightarrow \mathrm{H}_{m}$ is called $\delta$-positive if $\mathcal{L}-\delta \mathcal{I}(n, m)$ is completely positive.

Let $\mathcal{L}: \mathrm{H}_{n} \rightarrow \mathrm{H}_{m}$ be a real linear transformation. Since any $F \in \mathbb{C}^{n \times n}$ is of the form $F=X+\mathbf{i} Y$, where $X, Y \in \mathrm{H}_{n}$ and $(\mathbf{i})^{2}=-1$, it follows that $\mathbb{C}^{n \times n}$ is the complexification of $\mathrm{H}_{n}$. Hence $\mathcal{L}$ extends to linear operators $\hat{\mathcal{L}}: \mathbb{C}^{n \times n} \rightarrow \mathbb{C}^{m \times m}$ over $\mathbb{C}$ by letting $\hat{\mathcal{L}}(\mathbf{i} X)=\mathbf{i} \mathcal{L}(X)$ for $X \in \mathrm{H}_{n}$. We will identify $\hat{\mathcal{L}}$ with $\mathcal{L}$ and no confusion will arise. Note that for $U \in \mathbb{C}^{n \times n}$ we have that $\mathcal{L}\left(U^{*}\right)=\mathcal{L}(U)^{*}$. Associate with $\mathcal{L}$ the following block Hermitian matrix of dimension $m n$ :

$$
Z(\mathcal{L})=\left[\begin{array}{cccc}
\mathcal{L}\left(\mathbf{e}_{1} \mathbf{e}_{1}^{*}\right) & \mathcal{L}\left(\mathbf{e}_{1} \mathbf{e}_{2}^{*}\right) & \cdots & \mathcal{L}\left(\mathbf{e}_{1} \mathbf{e}_{n}^{*}\right) \\
\mathcal{L}\left(\mathbf{e}_{2} \mathbf{e}_{1}^{*}\right) & \mathcal{L}\left(\mathbf{e}_{1} \mathbf{e}_{2}^{*}\right) & \cdots & \mathcal{L}\left(\mathbf{e}_{2} \mathbf{e}_{n}^{*}\right) \\
\vdots & \vdots & \vdots & \vdots \\
\mathcal{L}\left(\mathbf{e}_{n} \mathbf{e}_{1}^{*}\right) & \mathcal{L}\left(\mathbf{e}_{n} \mathbf{e}_{2}^{*}\right) & \cdots & \mathcal{L}\left(\mathbf{e}_{n} \mathbf{e}_{n}^{*}\right)
\end{array}\right], \mathbf{e}_{i}=\left(\delta_{1 i}, \ldots, \delta_{n i}\right)^{\top}, i \in[n]
$$

Denote by $\lambda_{m n}(Z(\mathcal{L}))$ the smallest eigenvalue of $Z(\mathcal{L})$. The following lemma follows from Choi's characterization of completely positive operators [6, 13].

Lemma 8.2. Let $\delta \geq 0$. A real linear transformation $\mathcal{L}: H_{n} \rightarrow H_{m}$ is $\delta$-positive if and only if $\lambda_{m n}(Z(\mathcal{L})) \geq$ $\delta$.

Proof. Recall Choi's theorem [6] that $\mathcal{L}-\delta \mathcal{I}(n, m)$ is completely positive if and only if $\lambda_{m n}(Z(\mathcal{L}-\delta \mathcal{I}(n, m))) \geq$ 0 . Clearly, for $U \in \mathbb{C}^{n \times n}$ we have that $\mathcal{I}(n, m)(U)=(\operatorname{tr} U) I_{m}$. Hence $Z(\mathcal{I}(n, m))=I_{m n}$, and $Z(\mathcal{L}-$ $\delta \mathcal{I}(n, m))=Z(\mathcal{L})-\delta I_{m n}$. Thus $\lambda_{m n}(Z(\mathcal{L}-\delta \mathcal{I}(n, m)))=\lambda_{m n}(Z(\mathcal{L}))-\delta \geq 0$ if and ony if $\lambda_{m n}(Z(\mathcal{L})) \geq \delta$.

Corollary 8.3. Let $\delta \geq 0$ and assume that $\mathcal{L}: H_{n} \rightarrow H_{m}$ is $\delta$-positive. Then $\mathcal{L}$ is completely positive. In particular $\mathcal{I}(n, m) \in C P(n, m)$.

Let $\mathcal{C}: \mathbb{C}^{n \times n} \rightarrow \mathbb{C}^{m \times m}$ be a linear transformation over $\mathbb{C}$. Then $\mathcal{C}$ is called rationally represented if the entries each matrix $\mathcal{L}\left(\mathbf{e}_{i} \mathbf{e}_{j}^{*}\right)$ are Gaussian rationals, denoted as $\mathbb{Q}+\mathbf{i} \mathbb{Q}$. Assume that $\mathcal{C}$ is rationally represented. Denote by $\langle\mathcal{C}\rangle=\sum_{i, j=1}^{n}\left\langle\mathcal{L}\left(\mathbf{e}_{i} \mathbf{e}_{j}^{*}\right)\right\rangle$ the complexity of $\mathcal{C}$.

Theorem 8.4. Let $\mathcal{A}, \mathcal{B} \in C P(n, m)$ be rationally represented. Assume furthermore that $\mathcal{B}$ is $\delta$-positive for a given rational $\delta>0$. Then $\hat{\rho}(\mathcal{A}, \mathcal{B})=\rho(\mathcal{A}, \mathcal{B})$. Suppose that $\rho(\mathcal{A}, \mathcal{B})>0$. Then for any $\varepsilon \in(0,1) \cap \mathbb{Q}$ one can find $\tilde{\rho}(\mathcal{A}, \mathcal{B}) \in \mathbb{Q}_{++}$, in polynomial time in $\langle\mathcal{A}\rangle+\langle\mathcal{B}\rangle+\langle\delta\rangle+\langle\varepsilon\rangle$, such that

$$
\tilde{\rho}(\mathcal{A}, \mathcal{B}) \leq \rho(\mathcal{A}, \mathcal{B}) \leq(1+\varepsilon) \tilde{\rho}(\mathcal{A}, \mathcal{B})
$$

Proof. As $\mathcal{B}\left(I_{n}\right) \succeq \delta\left(\operatorname{tr} I_{n}\right) I_{m}=\delta n I_{m}$, we deduce that $r\left(\mathcal{A}, \mathcal{B}, I_{n}\right) \leq(n \delta)^{-1} \rho\left(\mathcal{A}\left(I_{n}\right)\right)<t_{0} \in \mathbb{Q}_{++}$. Hence $\rho(\mathcal{A}, \mathcal{B})<t_{0}$. As $\mathcal{B} \succ 0$ part 5 of Lemma 7.3 yields that $\hat{\rho}(A, B)=\rho(A, B)$. As $\hat{\rho}(\mathcal{A}, \mathcal{B})>0$ Lemma 7.4 yields that $\mathcal{A}^{\vee}\left(I_{m}\right) \succ 0$. Hence $\rho(\mathcal{A}, \mathcal{B})=\hat{\rho}(\mathcal{A}, \mathcal{B}) \geq \rho\left(\mathcal{A}^{\vee}\left(I_{m}\right)^{-1} \mathcal{B}^{\vee}\left(I_{m}\right)\right)^{-1}>s_{0} \in \mathbb{Q}_{++}$. Note that the values of $s_{0}, t_{0}$ are based on the eigenvalue computations of $\lambda(A, B)$, given by (22), whose approximation is polynomial 18 .

We now start a bisection problem as in the proof of Theorem 4.2. Suppose that we know that $\rho(\mathcal{A}, \mathcal{B}) \in$ $\left[s_{k}, t_{k}\right]$, where $s_{k}, t_{k} \in \mathbb{Q}_{++}$. Let $t=\frac{s_{k}+t_{k}}{2}$. Consider the minimum problem (27). If $\mu(t) \geq 2 \tau$ then $\rho(\mathcal{A}, \mathcal{B})>t$. and we let $s_{k+1}=t, t_{k+1}=t_{k}$. Assume now $\mu(t)<2 \tau$. We claim that

$$
\rho(\mathcal{A}, \mathcal{B}) \leq t+\frac{\sqrt{2 \tau}}{\delta} .
$$

Indeed, let $X(t), Z(t)$ and $W$ be defined as in (28). Let $\lambda_{1}(W) \geq \cdots \geq \lambda_{m}(W)$ be the $m$-eigenvalues of $W$. Then

$$
2 \tau>\|W\|^{2}=\langle W, W\rangle=\sum_{j=1}^{m} \lambda_{j}(W)^{2} \geq \lambda_{m}(W)^{2} .
$$


As $\sqrt{2 \tau} I_{m}+W \geq 0$ we obtain

$$
\left(t+\frac{\sqrt{2 \tau}}{\delta}\right) \mathcal{B}(X(t))-\mathcal{A}(X(t))=Z(t)+\frac{\sqrt{2 \tau}}{\delta} \mathcal{B}(X(t))+W \geq Z(t)+\frac{\sqrt{2 \tau}}{\delta} \delta I_{m}+W \geq 0 .
$$

Thus $r(\mathcal{A}, \mathcal{B}, X(t)) \leq t+\frac{\sqrt{2 \tau}}{\delta}$ which implies (31). Now choose $\tau=\frac{\left(s_{k}+t_{k}\right)^{2} \delta^{2}}{32}$. So in the case $\mu(t)<2 \tau$ we set $s_{k+1}=s_{k}, t_{k+1}=\frac{s_{k}+3 t_{k}}{4}$.

To conclude we showed that $\rho(\mathcal{A}, \mathcal{B}) \in\left[s_{k+1}, t_{k+1}\right]$, where $\left[s_{k+1}, t_{k+1}\right] \subset\left[s_{k}, t_{k}\right]$ and $\left|t_{k+1}-s_{k}\right| \leq \frac{3}{4}\left|t_{k}-s_{k}\right|$. Hence in polynomial time in $\langle A\rangle+\langle B\rangle+\langle\delta\rangle+\langle\varepsilon\rangle$ we get the approximation $\tilde{\rho}(\mathcal{A}, \mathcal{B}) \in \mathbb{Q}_{++}$satisfying (30).

\section{Minimal weak optimal solutions for CP-operators}

For $X \in \mathrm{H}_{+, n}$ we call range $X \subseteq \mathbb{C}^{n}$ the support of $X$, and denote supp $X=\operatorname{range} X$. So dim supp $X=$ rank $X$. Assume that $\mathcal{A}, \mathcal{B} \in C P(n, m)$. Suppose furthermore that $\hat{\rho}(\mathcal{A}, \mathcal{B})<\infty$. A weak optimal $Y \in \mathrm{H}_{+, n}$ is called minimal weak optimal if there is no weak optimal $X \in \mathrm{H}_{+, n} \backslash\{0\}$ such that $\operatorname{supp} X \subsetneq \operatorname{supp} Y$.

The following result is an analog of Theorem 5.1.

Theorem 9.1. Let $m, n$ be positive integers. Assume that $\mathcal{A}, \mathcal{B} \in C P(n, m)$. Suppose that $\hat{\rho}(\mathcal{A}, \mathcal{B}) \in(0, \infty)$.

(1) Assume that $Y \in H_{+, n} \backslash\{0\}$ is weak optimal. Then at least one of the eigenvalues of $(\mathcal{A}-\hat{\rho}(\mathcal{A}, \mathcal{B}) \mathcal{B})(Y)$ is zero.

(2) Assume that there exists a weak optimal $Y \in H_{+, n}$ whose rank is $\ell \geq 1$. Let $\mathbf{V}=$ range $Y$. Denote by $\mathcal{A}^{\prime}, \mathcal{B}^{\prime}: \mathrm{S}(\mathbf{V}) \rightarrow H_{m}$ the restrictions of $\mathcal{A}, \mathcal{B}$ to all $X \in H_{n}$ such that range $X \subseteq \mathbf{V}$. Then $\mathcal{A}^{\prime}, \mathcal{B}^{\prime}$ are $C P$-operators. If $\ell \geq m$ then $\operatorname{rank}\left(\mathcal{A}^{\prime}-\hat{\rho}(\mathcal{A}, \mathcal{B}) \mathcal{B}^{\prime}\right)<m^{2}$.

(3) A minimal weak optimal $Y$ has rank at most $m$.

(4) Assume that $Y$ is a minimal weak optimal with rank $m$. Then $Y$ satisfies (21). Furthermore $\operatorname{rank}\left(\mathcal{A}^{\prime}-\hat{\rho}(\mathcal{A}, \mathcal{B}) \mathcal{B}^{\prime}\right)=m^{2}-1$.

(5) Let $Y^{\prime}$ be a minimal weak optimal with rank $\ell<m$. Then there exists a minimal weak optimal $Y$, satisfying range $Y=$ range $Y^{\prime}$ with the following property: The matrix $(\mathcal{A}-\hat{\rho}(\mathcal{A}, \mathcal{B}) \mathcal{B})(Y)$ has at least $\ell$ zero eigenvalues.

Proof. (1) Let $\mathcal{D}(t)=\mathcal{A}-t \mathcal{B}$ and $t_{0}=\hat{\rho}(\mathcal{A}, \mathcal{B})$. As $r(\mathcal{A}, \mathcal{B}, Y)=t_{0}$ it follows that $\mathcal{D}\left(t_{0}\right)(Y) \leq 0$. Suppose to the contrary that $\mathcal{D}\left(t_{0}\right)(Y)<0$, As $t_{0}>0$ there exists $0 \leq t_{1}<t_{0}$ such that $\mathcal{D}\left(t_{1}\right)(Y) \leq 0$. Hence $r(\mathcal{A}, \mathcal{B}, Y) \leq t_{1}<t_{0}$ contrary to our assumption.

(2) Choose an orthonormal basis $\mathbf{g}_{1}, \ldots, \mathbf{g}_{\ell}$ of $\mathbf{V}$. Then $P_{\mathbf{V}}=\sum_{i=1}^{\ell} \mathbf{g}_{i} \mathbf{g}_{i}^{*}$ is the orthogonal projection on $\mathbf{V}$. Note that for each $X \in \mathrm{H}_{n}, P_{\mathbf{V}} X P_{\mathbf{V}} \in \mathrm{S}(\mathbf{V})$. (Observe that $P_{\mathbf{V}}^{*}=P_{\mathbf{V}}$.) Suppose that $\mathcal{C}: \mathrm{H}_{n} \rightarrow \mathrm{H}_{m}$ is a CPoperator given by (91). Define $\hat{\mathcal{C}}: \mathrm{H}_{n} \rightarrow \mathrm{H}_{m}$ by $\hat{\mathcal{C}}(X)=\mathcal{C}\left(P_{\mathbf{V}} X P_{\mathbf{V}}\right)$. Then $\hat{\mathcal{C}}(X)=\sum_{j=1}^{k}\left(T_{j} P_{\mathbf{V}}\right) X\left(T_{j} P_{\mathbf{V}}\right)^{*}$. So $\hat{\mathcal{C}}$ is also a CP-operator. Hence $\mathcal{A}^{\prime}, \mathcal{B}^{\prime}$ are CP-operators. By choosing an orthonormal basis $\mathbf{g}_{1}, \ldots, \mathbf{g}_{\ell}$ of $\mathbf{V}$ we can identify $\mathrm{S}(\mathbf{V})$ with $\mathrm{H}_{\ell}$. So $\mathcal{A}^{\prime}, \mathcal{B}^{\prime}: \mathrm{H}_{\ell} \rightarrow \mathrm{H}_{m}$. Recall that $\operatorname{dim} \mathrm{H}_{\ell}=\ell^{2}, \operatorname{dim} \mathrm{H}_{m}=m^{2}$. Clearly, $\hat{\rho}\left(\mathcal{A}^{\prime}, \mathcal{B}^{\prime}\right)=\hat{\rho}(\mathcal{A}, \mathcal{B})$. By abusing the notation we assume that an optimal $Y$ is in $\mathrm{H}_{+, \ell}$.

Assume that $\ell \geq m$. Let $\mathcal{C}(t)=\mathcal{A}^{\prime}-t \mathcal{B}^{\prime}: \mathrm{H}_{\ell} \rightarrow \mathrm{H}_{m}$. Thus $\mathcal{C}\left(t_{0}\right)(Y)=-W$ for some $W \in \mathrm{H}_{+, m}$. Clearly, $\operatorname{rank} \mathcal{C}\left(t_{0}\right) \leq m^{2}$. Assume to the contrary that $\operatorname{rank} \mathcal{C}\left(t_{0}\right)=m^{2}$. Hence the exists an $m^{2}$ dimensional subspace $\mathbf{W} \subseteq \mathrm{H}_{\ell}$ such that $\mathcal{C}\left(t_{0}\right) \mid \mathbf{W}$ is an invertible operator. Let $\mathcal{A}_{1}, \mathcal{B}_{1}$ and $\mathcal{A}_{2}, \mathcal{B}_{2}$ be the restrictions of $\mathcal{A}^{\prime}, \mathcal{B}^{\prime}$ to $\mathbf{W}$ and $\mathbf{W}^{\perp}$ respectively. Define $\mathcal{C}_{1}(t)=\mathcal{A}_{1}-t \mathcal{B}_{1}, \mathcal{C}_{2}(t)=\mathcal{A}_{2}-t \mathcal{B}_{2}$. As $\mathcal{C}_{1}\left(t_{0}\right)$ is invertible, it follows that there exists $\varepsilon>0$ such that $C_{1}(t): \mathbf{W} \rightarrow \mathrm{H}_{m}$ is invertible for $t \in\left[t_{0}-\varepsilon, t_{0}+\varepsilon\right]$. Let $Y=Y_{1}+Y_{2}$, where $Y_{1} \in \mathbf{W}, Y_{2} \in \mathbf{W}^{\perp}$. Assume that $Y_{1}(t)=-\mathcal{C}_{1}(t)^{-1}\left(\mathcal{C}_{2}(t)\left(Y_{2}\right)+W\right)$. So $Y_{1}\left(t_{0}\right)=Y_{1}$. Hence $\mathcal{C}(t)\left(Y_{1}(t)+Y_{2}\right)=-W$. As rank $\left(Y_{1}+Y_{2}\right)=\ell$ it follows that there exits $t_{0}-\varepsilon<t_{1}<t_{0}$ such that $Y_{1}(t)+Y_{2} \in \mathrm{H}_{+, \ell}$ and $\operatorname{rank}\left(Y_{1}(t)+Y_{2}\right)=\ell$. Therefore $r\left(\mathcal{A}, \mathcal{B}, Y_{1}(t)+Y_{2}\right) \leq t_{1}<\hat{\rho}(\mathcal{A}, \mathcal{B})$, which contradicts the definition of $\hat{\rho}(\mathcal{A}, \mathcal{B})$.

(3) Assume to the contrary that $Y$ is a minimal weak optimal with rank $\ell>m$. Assume as in 2 that we restricted ourselves to $\mathcal{A}^{\prime}, \mathcal{B}^{\prime}: \mathrm{H}_{\ell} \rightarrow \mathrm{H}_{m}$. Let $t_{0}$ and $\mathcal{C}\left(t_{0}\right)$ be defined as above. As $\ell>m$ it follows that $\operatorname{dim} \mathrm{H}_{\ell}=\ell^{2} \geq(m+1)^{2}=m^{2}+2 m+1$. So $\operatorname{dim} \operatorname{ker} \mathcal{C}\left(t_{0}\right) \geq 2 m+1 \geq 2$. Hence there exists an indefinite matrix $X \in \mathrm{H}_{\ell}$, with at least one positive and one negative eigenvalue such that $\mathcal{C}\left(t_{0}\right)(X)=0$. Let $s \in[0, \infty]$ an consider the matrix $Y(s)=Y+s X$. For $s=0 Y \in \mathrm{H}_{++, \ell}$. For $s \gg 1 Y(s)$ has a negative eigenvalue. Hence there exists $s_{0}>0$ such that $Y\left(s_{0}\right) \in \mathrm{H}_{+, \ell}$ and at least one eigenvalue of $Y\left(s_{0}\right)$ is 0 . Note that $Y\left(s_{0}\right) \neq 0$ as $Y$ can not be proportional to $X$. As $\mathcal{C}\left(t_{0}\right)\left(Y\left(s_{0}\right)\right)=\mathcal{C}\left(t_{0}\right)(Y)=-W$ it follows that $Y\left(s_{0}\right)$ is optimal. As rank $Y\left(s_{0}\right)<\ell$, we deduce that $Y$ is not minimal weak optimal contrary to our assumptions. 
(4) Let $Y$ be a minimal weak optimal of rank $m$. Assume as in 2 that we restricted ourselves to $\mathcal{A}^{\prime}, \mathcal{B}^{\prime}$ : $\mathrm{H}_{m} \rightarrow \mathrm{H}_{m}$. Part (2) yields that $\operatorname{rank} \mathcal{C}\left(t_{0}\right)<m^{2}$. Let $X \neq 0$ satisfy $C\left(t_{0}\right)(X)=0$. If $X$ is indefinite, as in the proof of (2), we deduce that $Y$ is not a minimal weak optimal, contrary to our assumption. If $X$ or $-X$ is positive semidefinite then either $X$ or $-X$ is a GPF-vector. We claim that $Y$ is proportional to $X$. Otherwise, assuming that $X$ is positive semidefine, by considering $Y-s X$ we deduce that $Y$ is not optimal. Hence $Y$ is a $\mathrm{GPF}$-vector. Moreover, the above $\operatorname{arguments}$ yield that $\operatorname{dim} \operatorname{ker} \mathcal{C}\left(t_{0}\right)=1$, i.e. $\operatorname{rank} \mathcal{C}\left(t_{0}\right)=m^{2}-1$.

(5) Assume that $Y^{\prime} \in \mathrm{H}_{+, n}$ is a minimal weak optimal with rank $\ell<m$. So $(\mathcal{A}-\rho(A, B) \mathcal{B})\left(Y^{\prime}\right) \leq 0$. By part 1 we know that $(\mathcal{A}-\rho(A, B) \mathcal{B})\left(Y^{\prime}\right)$ has at least one zro eigenvalue. If $\ell=1$ the claim 5 of the theorem trivially holds.

Assume that $\ell>1$. Consider all minimal weak optimal $\tilde{Y}$ such that range $\tilde{Y}=\operatorname{range} Y^{\prime}$. Let $Y$ be a minimal weak optimal satisfying: range $Y=\operatorname{range} Y^{\prime}$ and $(\mathcal{A}-\rho(A, B) \mathcal{B})(Y)$ has the maximum number of zero eigenvalues. Assume that this maximum is $p$. We claim that $p \geq \ell$. Suppose not. As in the proof of part (2) we restrict ourselves to $\mathcal{A}^{\prime}, \mathcal{B}^{\prime}: \mathrm{H}_{\ell} \rightarrow \mathrm{H}_{m}$. So $t_{0}=\rho\left(\mathcal{A}^{\prime}, \mathcal{B}^{\prime}\right)$. By abusing the notation we assume that $Y^{\prime}, \tilde{Y}, Y \in \mathrm{H}_{+, \ell}$. Let $W=-C\left(t_{0}\right)(Y)$. Then $\mathbb{C}^{m}=\mathbf{U}_{1} \oplus \mathbf{U}_{2}$, where $\mathbf{U}_{1}=$ range $W, \mathbf{U}_{2}=\mathbf{U}_{1}^{\perp}=\operatorname{ker} W$. Note that $W \mid \mathbf{U}_{1}$ positive definite. Let $P_{\mathbf{U}_{2}}$ be the orthogonal projection of $\mathbb{C}^{m}$ on $\mathbf{U}_{2}$. Let $\tilde{\mathcal{A}}, \tilde{\mathcal{B}}: \mathrm{H}_{\ell} \rightarrow \mathrm{H}_{p}$, where we identify $\mathrm{H}_{p}$ with $P_{\mathbf{U}_{2}} \mathrm{H}_{m} P_{\mathbf{U}_{2}}$ and $\tilde{\mathcal{A}}, \tilde{\mathcal{B}}$ with $P_{\mathbf{U}_{2}} \mathcal{A} P_{\mathbf{U}_{2}}, P_{\mathbf{U}_{2}} \mathcal{B} P_{\mathbf{U}_{2}}$ respectively. Clearly, $\hat{\rho}(\tilde{\mathcal{A}}, \tilde{\mathcal{B}}) \leq$ $\hat{\rho}\left(\mathcal{A}^{\prime}, \mathcal{B}^{\prime}\right)$. As in the proof of part (5) of Theorem 5.1 we claim that $\hat{\rho}(\tilde{\mathcal{A}}, \tilde{\mathcal{B}})=\hat{\rho}\left(\mathcal{A}^{\prime}, \mathcal{B}^{\prime}\right)$. Suppose not. Then there exists $U \in \mathrm{H}_{+, \ell} \backslash\{0\}$ such that $r(\tilde{\mathcal{A}}, \tilde{\mathcal{B}}, U)=t_{1}<t_{0}$. Clearly, $Y$ and $U$ are linearly independent. As $Y \in \mathrm{H}_{++, \ell}$ we can assume that $Y \geq U$. Let $Y(f)=(1-f) Y+f U$. As in the proof of part (5) of Theorem 5.1 we claim that there exists $\varepsilon>0$ such that $r(\tilde{\mathcal{A}}, \tilde{\mathcal{B}},(1-f) Y+f U)) \leq t_{0}-\varepsilon f$ for $f \in[0,1]$. We can choose $\varepsilon=\left(t_{0}-t_{1}\right) \lambda^{\prime}$, where $\lambda^{\prime}$ is the smallest positive eigenvalue of $\tilde{\mathcal{B}}(Y)^{-1} \tilde{\mathcal{B}}(U)$. (Note that the eigenvalues of $\tilde{\mathcal{B}}(Y)^{-1} \tilde{\mathcal{B}}(U)$ are the eigenvalues of the Hermitian matrix $\tilde{\mathcal{B}}(Y)^{-\frac{1}{2}} \tilde{\mathcal{B}}(U) \tilde{\mathcal{B}}(Y)^{-\frac{1}{2}}$.) We now claim that there exists small positive $\delta$ such that for we have the inequality

$$
\left.r\left(\mathcal{A}^{\prime}, \mathcal{B}^{\prime},(1-f) Y+f U\right)\right) \leq t_{0}-\frac{1}{2} \varepsilon f, \quad f \in[0, \delta] .
$$

For that it is enough to show that $\lambda\left(\mathcal{A}^{\prime}((1-f) Y+f U), \mathcal{B}^{\prime}((1-f) Y+f U)\right) \leq t_{0}-\frac{1}{2} \varepsilon f$ for $f \in[0, \delta]$. We use the first variation formula for a geometrically simple eigenvalue $t_{0}$ of the Rayleigh ratio on the right-hand side of (22), where $\left.A=A(f)=\mathcal{A}^{\prime}((1-f) Y+f U)\right)$ and $B=B(f)=\mathcal{B}^{\prime}((1-f) Y+f U)$, as a function in $f$. The standard variation formula for a geometrically simple eigenvalue [12, §3.8] yields that it is enough to consider the first variation of the Rayleigh quotient given by the right-hand side of (22) for $\mathbf{x} \in \mathbf{U}_{2} \backslash\{\mathbf{0}\}$. This is equivalent to consider $\lambda(\tilde{\mathcal{A}}((1-f) Y+f U), \tilde{\mathcal{B}}((1-f) Y+f U))$. The inequality $\lambda(\tilde{\mathcal{A}}((1-f) Y+f U), \tilde{\mathcal{B}}((1-f) Y+f U)) \leq t_{0}-\varepsilon f$ for $f \in[0, \delta]$ yields that

$$
\left.\frac{d}{d f} \lambda\left(\mathcal{A}^{\prime}((1-f) Y+f U), \mathcal{B}^{\prime}((1-f) Y+f U)\right)\right|_{f=0^{+}} \leq-\varepsilon .
$$

Recall Rellich's theorem that the eigenvalues of analytic functions of Hermitian matrices are analytic in the neighborhood of $\mathbb{R}[12,4.17]$, (when we do not insist on ordering of the eigenvalues). Hence $\lambda\left(\mathcal{A}^{\prime}((1-f) Y+\right.$ $\left.f U), \mathcal{B}^{\prime}((1-f) Y+f U)\right)$ is analytic in $[0, \delta]$ for some $\delta>0$. That is, it has convergent Taylor series at $f=0$. In particular it is in the class $C^{2}[0, \delta]$. Hence (33) yields (32) for small enough positive $\delta$.

Clearly, (32) contradicts our assumption that $\hat{\rho}\left(\mathcal{A}^{\prime}, \mathcal{B}^{\prime}\right)=t_{0}$. Therefore $\hat{\rho}(\tilde{\mathcal{A}}, \tilde{\mathcal{B}})=t_{0}=r(\tilde{\mathcal{A}}, \tilde{\mathcal{B}}, Y)$. Let $\tilde{\mathcal{C}}(t)=\tilde{\mathcal{A}}-t \tilde{\mathcal{B}}$. So $\tilde{\mathcal{C}}\left(t_{0}\right): \mathrm{H}_{\ell} \rightarrow \mathrm{H}_{p}$. Our assumption that $\ell>p$ yields that $\operatorname{ker} \tilde{\mathcal{C}}\left(t_{0}\right) \geq \ell^{2}-p^{2} \geq 3$. In particular, there exists an indefinite $\tilde{X} \in \mathrm{H}_{\ell}$ such that $\tilde{\mathcal{C}}\left(t_{0}\right)(\tilde{X})=0$. Identify $\tilde{X}$ with $X \in \mathrm{H}_{n}$, were range $X \subseteq$ range $Y$. The assumption that $\tilde{\mathcal{C}}\left(t_{0}\right)(\tilde{X})=0$ is equivalent to range $\mathcal{C}\left(t_{0}\right)(X) \subseteq$ range $\mathcal{C}\left(t_{0}\right)(Y)=$ range $W$. Let $Y(s)=Y+s X$. Then $\mathcal{C}\left(t_{0}\right)(Y(s))=-W+s \mathcal{C}\left(t_{0}\right)(X)$. As range $\mathcal{C}\left(t_{0}\right)(X) \subseteq$ range $\mathcal{C}\left(t_{0}\right)(Y)=$ range $W$ it follows that $-W+s \mathcal{C}\left(t_{0}\right)(X) \leq 0$ for $s \geq 0$ if and only if $s \in\left[0, s_{1}\right]$, where $s_{1} \in(0, \infty]$. Let $s_{0}>$ be the largest $s>0$ such that $Y(s) \geq 0$. So rank $Y\left(s_{0}\right) \in[\ell-1]$. Note that $Y(s)$ is optimal for $s \in\left[0, \min \left(s_{0}, s_{1}\right)\right]$. We claim that $s_{1}<s_{0}$. Suppose not. Then $Y\left(s_{0}\right)$ is weak optimal, range $Y\left(s_{0}\right) \subset$ range $Y$ and rank $Y\left(s_{0}\right) \leq \ell-1$. Hence $Y$ is not minimal weak optimal, contrary to our assumptions. Consider a minimal weak optimal $Y\left(s_{1}\right)$. Note that $\operatorname{rank}\left(-W+s_{1} \mathcal{C}\left(t_{0}\right)(X)\right)<\operatorname{rank}-W=m-p$. Hence $\operatorname{ker} \mathcal{C}\left(t_{0}\right)\left(Y\left(s_{1}\right) \geq p+1\right.$, contrary to the choice of $Y$. Therefore $p \geq \ell$. 
Acknowledgements. The author thanks the referee for his comments. Shmuel Friedland was partially supported by Simons collaboration grant for mathematicians.

\section{REFERENCES}

[1] C. Avin, M. Borokhovich, Y. Haddad, E. Kantor, Z. Lotker, M. Parter, D. Peleg, Generalized Perron-Frobenius Theorem for Multiple Choice Matrices, and Applications, Proc. 24rd ACM-SIAM SODA, 478-497, 2013. Zbl 1422.90013, MR3186769

[2] C. Avin, M. Borokhovich, Y. Haddad, E. Kantor, Z. Lotker, M. Parter, D. Peleg, Generalized Perron-Frobenius Theorem for Nonsquare Matrices, arXiv:1308.5915.

[3] A. Berman and R.J. Plemmons, Nonnegative Matrices in Mathematical Sciences, Academic Press, New York 1979. Zbl 0484.15016, MR0544666

[4] G. Boutry, M. Elad, G.H. Golub, and P. Milanfar, The generalized eigenvalue problem for nonsquare pencils using a minimal perturbation approach, SIAM J. Matrix Anal. Appl., 27( 2005), 582-601. Zbl 1100.65035, MR2179690

[5] S. Boyd and L. Vandenberghe, Convex Optimization, Cambridge Univ. Press, NY, 2004. Zbl 1058.90049, MR2061575

[6] M.D. Choi, Completely positive linear maps on complex matrices, Linear Algebra and Appl. 10 (1975), 285-290. Zbl 0327.15018, MR0376726

[7] D. Chu and G. H. Golub, On a generalized eigenvalue problem for nonsquare pencils, SIAM J. Matrix Analysis Applications, 28 (2006), 770-787. Zbl 1128.15004, MR2262980

[8] L. Collatz, Einschließungssatz für die charakteristischen Zahlen von Matrizen, Mathematische Zeitschrift 48 (1942), 221226. Zbl 0027.00604, MR0008590

[9] I. Erdélyi, On the matrix equation $A x=\lambda B x$, J. Math. Anal. \& Applic. 17 (1967), 119-132. Zbl 0153.04902, MR0202734

[10] S. Friedland, Characterization of spectral radius of positive operators, Linear Algebra Appl. 134 (1990), 93-105. Zbl 0707.15005, MR1060012

[11] S. Friedland, Characterizations of spectral radius of positive operators on $C^{*}$ algebras, J. Funct. Anal. 97 (1991), 64-70. Zbl 0745.47024, MR1105655

[12] S. Friedland, Matrices: Algebra, Analysis and Applications, World Scientific, 596 pp., 2015, Singapore. Zbl 1337.15002, MR3467205

[13] S. Friedland and R. Loewy, On the extreme points of quantum channels, Linear Algebra Appl. 498 (2016), 553-573. Zbl 1334.15086, MR3478578

[14] G.F. Frobenius, Über Matrizen aus positiven Elementen, 1, Preussiche Akademie der Wissenschaften zu Berlin, 471-476, 1908. JFM 39.0213.03

[15] G.F. Frobenius, Über Matrizen aus positiven Elementen, 2, Preussiche Akademie der Wissenschaften zu Berlin, 514-518, 1909. JFM 40.0202.02

[16] G.F. Frobenius, Über Matrizen aus nicht negativen Elementen, Preussiche Akademie der Wissenschaften zu Berlin, 456-477, 1912. JFM 43.0204.09,

[17] F.R. Gantmacher, The Theory of Matrices, Vol. I and II, Chelsea Publ. Co., New York 1959. Zbl 0927.15001, MR0107649

[18] G.H. Golub and C.F. Van Loan, Matrix Computations, Johns Hopkins Studies in the Mathematical Sciences, Fourth Edition, 2013. Zbl 1268.65037, MR3024913

[19] M. Grötzschel, L. Lovász, and A. Schrijver, Geometric Algorithms and Combinatorial Optimization, Springer, Berlin, 1988. Zbl 0634.05001, MR0936633

[20] M.B. Hastings, Superadditivity of communication capacity using entangled inputs, Nature Phys., 5 (2009), $255-257$.

[21] A.S. Holevo, The additivity problem in quantum information theory, in Proc. Int. Congr. Math. Eur. Math. Soc., Zurich, Switzerland, 2006, vol. III, pp. 999-1018. Zbl 1100.94007, MR2275716

[22] A.S. Holevo, Quantum Systems, Channels, Information, de Gruyter, Berlin, 2012. Zbl 1332.81003, MR2986302

[23] R.A. Horn and C.R. Johnson, Matrix Analysis, 2nd ed., Cambridge University Press, Cambridge, 2013. Zbl 1267.15001, MR2978290

[24] S. Karlin, Positive operators, J. Math. Mech. 8 (1959), 905-937. Zbl 0087.11002, MR0114138

[25] M.G. Krein and M.A. Rutman, Linear operators leaving invariant cone in a Banach space, Uspekhi Mat. Nauk 3 (1948), 3-95, Amer. Math. Soc. Trans. 26, 1950. Zbl 0030.12902, MR0038008

[26] L. Lovász, An Algorithmic Theory of Numbers, Graphs and Convexity, CBMS-NSF Regional Conference Series in Applied Mathematics, SIAM, 1986. Zbl 0606.68039, MR0861822

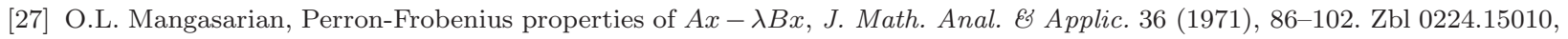
MR0285555

[28] C.B. Mendl and M.M. Wolf, Unital quantum channels - convex structure and revivals of Birkhoffs theorem, Commun. Math. Phys. 289 (2009), 1057-1086. Zbl 1167.81011, MR2511660

[29] C.D. Meyer. Matrix Analysis and Applied Linear Algebra, SIAM, 2000. Zbl 0962.15001, MR1777382

[30] H. Minc, Nonnegative Matrices, Wiley-Interscience, New York, 1988. Zbl 0638.15008, MR0932967

[31] O. Perron, Zur theorie der Matrices, Mathematische Annalen, 64 (1907), 248-263. JFM 38.0202.01, MR1511438

[32] D. Petz, Quantum Information Theory and Quantum Statistics, Springer, NewYork, 2008. Zbl 1145.81002, MR2363070

[33] S.U. Pillai, T. Suel, and S. Cha, The Perron-Frobenius theorem, IEEE Signal Process.Mag., 22 (2005), $62-75$.

[34] H.H. Schaeffer, Banach Lattices and Positive Operators. Springer, New York, 1974. Zbl 0296.47023, MR0423039

[35] E. Seneta, Non-Negative Matrices and Markov Chains, 2nd ed. Springer, New York, 1981. Zbl 0471.60001, MR0719544

[36] M.E. Shirokov, On the structure of optimal sets for a quantum channel, Probl. Inf. Transmiss., 42 (2006), 23-40. Zbl 1237.94039, MR2278809 
[37] P.W. Shor, Additivity of the classical capacity of entanglement- breaking quantum channels, J. Math. Phys., 43 (2002), 4334-4340. Zbl 1060.94004, MR1924442

[38] R. Srikant, The Mathematics of Internet Congestion Control, Birkhauser, Boston, 2003. Zbl 1086.68018, MR2018967

[39] H. Wielandt, Unzerlegbare nicht-negative Matrizen, Mathematische Zeitschrift 52 (1950), 642-648. Zbl 0035.29101, MR0035265

Department of Mathematics, Statistics and Computer Science, University of Illinois, Chicago, IL, 60607-7045, EMAIL: FRIEDLAN@UIC.EDU 